\title{
Utilization of cyclic amides as masked aldehyde equivalents in reductive amination reactions
}

\author{
Robin J. Prince, ${ }^{\dagger}$ Fang Gao, ${ }^{\dagger}$ Jessica E. Pazienza, Isaac E. Marx, Jurgen \\ Schulz, and Brian T. Hopkins \\ Biotherapeutics and Medicinal Sciences, Biogen Inc. \\ 225 Binney Street, Cambridge MA, 02142 \\ SUPPORTING INFORMATION \\ Table of Contents
}

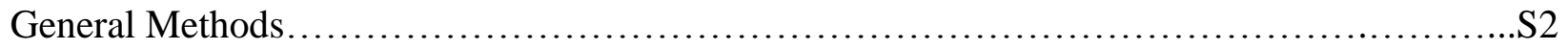

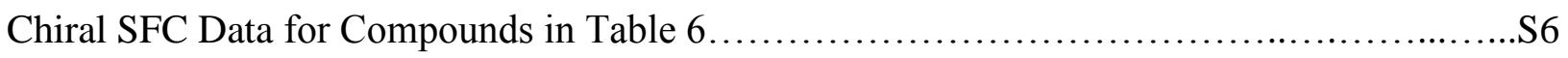

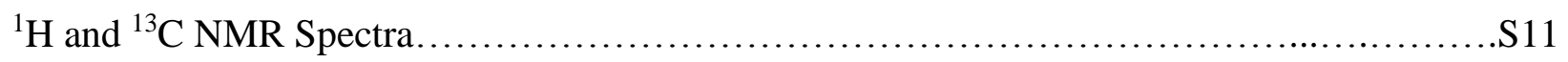




\section{GENERAL METHODS}

\section{$\underline{\mathrm{LC} / \mathrm{MS}}$}

The sample was dissolved in a suitable solvent such as $\mathrm{MeCN}$, DMSO, or MeOH and was injected directly into the column using an automated sample handler. The analysis was performed using one of the following methods:

\section{$\underline{\text { Analytical LCMS }}$}

Acidic LCMS: Conducted on a Shimadzu 2010 Series, Shimadzu 2020 Series, or Waters Acquity UPLC BEH. (MS ionization: ESI) instrument equipped with a C18 column (2.1 mm x $30 \mathrm{~mm}, 3.0 \mathrm{~mm}$ or $2.1 \mathrm{~mm}$ x $50 \mathrm{~mm}, \mathrm{C} 18,1.7 \mu \mathrm{m})$, eluting with $1.5 \mathrm{~mL} / 4 \mathrm{~L}$ TFA in water (solvent $\mathrm{A}$ ) and $0.75 \mathrm{~mL} / 4 \mathrm{~L}$ TFA in acetonitrile (solvent $\mathrm{B}$ ) using the methods below:

1.5-minute methods:

General method: using the following elution gradient 5\% - 95\% (solvent B) over 0.7 minutes and holding at $95 \%$ for 0.4 minutes at a flow rate of $1.5 \mathrm{~mL} /$ minute. Wavelength: UV $220 \mathrm{~nm}$ and $254 \mathrm{~nm}$.

2-minute methods:

Method A: using the following elution gradient $0 \%-60 \%$ (solvent B) over 0.9 minutes and holding at $60 \%$ for 0.6 minutes at a flow rate of $1.2 \mathrm{~mL} /$ minute. Wavelength: UV $220 \mathrm{~nm}$ and $254 \mathrm{~nm}$.

Method B: using the following elution gradient 10\% - 80\% (solvent B) over 0.9 minutes and holding at $60 \%$ for 0.6 minutes at a flow rate of $1.2 \mathrm{~mL} /$ minute. Wavelength: UV $220 \mathrm{~nm}$ and $254 \mathrm{~nm}$.

Method C: using the following elution gradient 30\% - 90\% (solvent B) over 0.9 minutes and holding at $60 \%$ for 0.6 minutes at a flow rate of $1.2 \mathrm{~mL} /$ minute. Wavelength: UV $220 \mathrm{~nm}$ and $254 \mathrm{~nm}$. 


\section{5-minute method:}

Initial conditions, solvent A-95\% : solvent B-5\%; hold at initial from $0.0-0.1 \mathrm{~min}$; Linear Ramp to solvent A-5\% : solvent B-95\% between 0.1 - 3.25 min; hold at solvent A-5\% : solvent B-95\% between 3.25 - 3.5 min. Diode array/MS detection.

4-minute methods:

Method A: using the following elution gradient 0\% - 60\% (solvent B) over 3 minutes and holding at $60 \%$ for 0.5 minutes at a flow rate of $0.8 \mathrm{~mL} /$ minute. Wavelength: UV $220 \mathrm{~nm}$ and $254 \mathrm{~nm}$.

Method B: using the following elution gradient 10\%-80\% (solvent B) over 3 minutes and holding at $60 \%$ for 0.5 minutes at a flow rate of $0.8 \mathrm{~mL} /$ minute. Wavelength: UV $220 \mathrm{~nm}$ and $254 \mathrm{~nm}$.

Method C: using the following elution gradient 30\%-90\% (solvent B) over 3 minutes and holding at $60 \%$ for 0.5 minutes at a flow rate of $0.8 \mathrm{~mL} /$ minute. Wavelength: UV $220 \mathrm{~nm}$ and $254 \mathrm{~nm}$.

7-minute methods:

Method A: using the following elution gradient 0\%-60\% (solvent B) over 6 minutes and holding at $60 \%$ for 0.5 minutes at a flow rate of $0.8 \mathrm{~mL} /$ minute. Wavelength: UV $220 \mathrm{~nm}$ and $254 \mathrm{~nm}$.

Method B: using the following elution gradient 10\% - 80\% (solvent B) over 6 minutes and holding at $60 \%$ for 0.5 minutes at a flow rate of $0.8 \mathrm{~mL} /$ minute. Wavelength: UV $220 \mathrm{~nm}$ and $254 \mathrm{~nm}$.

Method C: using the following elution gradient 30\% - 90\% (solvent B) over 6 minutes and holding at $60 \%$ for 0.5 minutes at a flow rate of $0.8 \mathrm{~mL} /$ minute. Wavelength: UV $220 \mathrm{~nm}$ and $254 \mathrm{~nm}$.

Basic LCMS: Conducted on a Shimadzu 2020 Series or Waters Acquity UPLC BEH (MS ionization: ESI) instrument equipped with XBridge Shield RP18, $5 \mu \mathrm{m}$ column $(2.1 \mathrm{~mm} \mathrm{x}$ 
$30 \mathrm{~mm}, 3.0 \mathrm{~mm}$ i.d.) or $2.1 \mathrm{~mm}$ x $50 \mathrm{~mm}, \mathrm{C} 18,1.7 \mu \mathrm{m}$ column, eluting with $2 \mathrm{~mL} / 4 \mathrm{~L} \mathrm{NH}_{3} \cdot \mathrm{H}_{2} \mathrm{O}$ in water (solvent A) and acetonitrile (solvent B) using the methods below:

3-minute methods:

Method A: using the following elution gradient $0 \%-60 \%$ (solvent B) over 2 minutes and holding at $60 \%$ for 0.48 minutes at a flow rate of $1 \mathrm{~mL} /$ minute. Wavelength: UV $220 \mathrm{~nm}$ and $254 \mathrm{~nm}$.

Method B: using the following elution gradient 10\% - 80\% (solvent B) over 2 minutes and holding at $60 \%$ for 0.48 minutes at a flow rate of $1 \mathrm{~mL} /$ minute. Wavelength: UV $220 \mathrm{~nm}$ and $254 \mathrm{~nm}$.

Method C: using the following elution gradient 30\% - 90\% (solvent B) over 2 minutes and holding at $60 \%$ for 0.48 minutes at a flow rate of $1 \mathrm{~mL} /$ minute. Wavelength: UV $220 \mathrm{~nm}$ and $254 \mathrm{~nm}$.

\section{5-minute method:}

Initial conditions, solvent A-95\% : solvent B-5\%; hold at initial from 0.0 - 0.1 min; Linear Ramp to solvent A-5\% : solvent B-95\% between $0.1-3.25$ min; hold at solvent A-5\% : solvent B-95\% between 3.25 - 3.5 min. Diode array/MS detection.

7-minute methods:

Method A: using the following elution gradient 0\% - 60\% (solvent B) over 6 minutes and holding at $60 \%$ for 0.5 minutes at a flow rate of $0.8 \mathrm{~mL} /$ minute. Wavelength: UV $220 \mathrm{~nm}$ and $254 \mathrm{~nm}$.

Method B: using the following elution gradient 10\% - 80\% (solvent B) over 6 minutes and holding at $60 \%$ for 0.5 minutes at a flow rate of $0.8 \mathrm{~mL} /$ minute. Wavelength: UV $220 \mathrm{~nm}$ and $254 \mathrm{~nm}$.

Method C: using the following elution gradient 30\% - 90\% (solvent B) over 6 minutes and holding at $60 \%$ for 0.5 minutes at a flow rate of $0.8 \mathrm{~mL} /$ minute. Wavelength: UV $220 \mathrm{~nm}$ and $254 \mathrm{~nm}$. 


\section{Preparative HPLC purification}

General Method: Preparative HPLC was performed on a Gilson UV/VIS-156 with UV detection at 220/254 $\mathrm{nm}$ Gilson 281 automatic collection.

Acidic condition: Two acid grading systems used: hydrochloric acid and formic acid.

Method A: hydrochloric acid: YMC-Actus Triart C18 150 x $30 \mathrm{~mm}$ x $5 \mu \mathrm{m}$, Gradient used 0 - $100 \%$ acetonitrile with water and corresponding acid $(0.05 \% \mathrm{HCl})$.

Method B: formic acid: Phenomenex Synergi C18 150 x $30 \mathrm{~mm}$ x $4 \mu \mathrm{m}$, Gradient used 0 - $100 \%$ acetonitrile with water and corresponding acid $(0.225 \%$ formic acid $)$, the gradient shape was optimized for individual separations.

Neutral condition: Xtimate C18 150 x 25 mm x $5 \mu \mathrm{m}$, Gradient used 0 - 100\% (water (10 $\left.\left.\mathrm{mM} \mathrm{NH}_{4} \mathrm{HCO}_{3}\right)-\mathrm{ACN}\right)$, the gradient shape was optimized for individual separations.

Basic condition: Waters Xbridge Prep OBD C18 150 x 30 x $10 \mu \mathrm{m}$, Gradient used 0 $100 \%$ water $\left(0.04 \% \mathrm{NH}_{3} \mathrm{H}_{2} \mathrm{O}+10 \mathrm{mM} \mathrm{NH} \mathrm{NHCO}_{3}\right)$-acetonitrile, the gradient shape was optimized for individual separations.

\section{Preparative HPLC-MS purification}

\section{Columns used:}

Acid: Waters SunFire Prep, C18 $5 \mu \mathrm{m}$, OBD 19 x 100 mm

Base: Waters XSelect CSH Prep, C18 5 m, OBD 19 x 100 mm

Gradient Profile: 12 min Run: Initial conditions: A-95\% : B-5\%; hold at initial from 0.0 0.5 min; linear ramp from A-5\% to variable B-\% (typical range is from B-40\% to B-75\%) between 0.5 - $7.5 \mathrm{~min}$; linear ramp from B-\% to B-95\% from 7.5 - $8.0 \mathrm{~min}$; hold at A-5\% : B95\% between 8.0 - $10.0 \mathrm{~min}$; end of $\mathrm{DAD} / \mathrm{MS}$ detection; linear ramp down to initial conditions between 10.0 - $10.5 \mathrm{~min}$ and hold at initial for $1.5 \mathrm{~min}$.

Mobile Phase: Acid: A: 0.1\% trifluoroacetic acid in water (v/v); Mobile phase B: $0.1 \%$ trifluoroacetic acid in acetonitrile (v/v). Base: A: 0.1\% ammonia in water (v/v); Mobile phase B: $0.1 \%$ ammonia in acetonitrile (v/v). 
Chiral SFC Traces for Determining Enantiospecificity<smiles>COc1ccc(CN2CCC[C@H](Nc3ccc(C(F)(F)F)cc3)C2=O)cc1</smiles>

8a; Enantio-specificity determination: Thar SFC
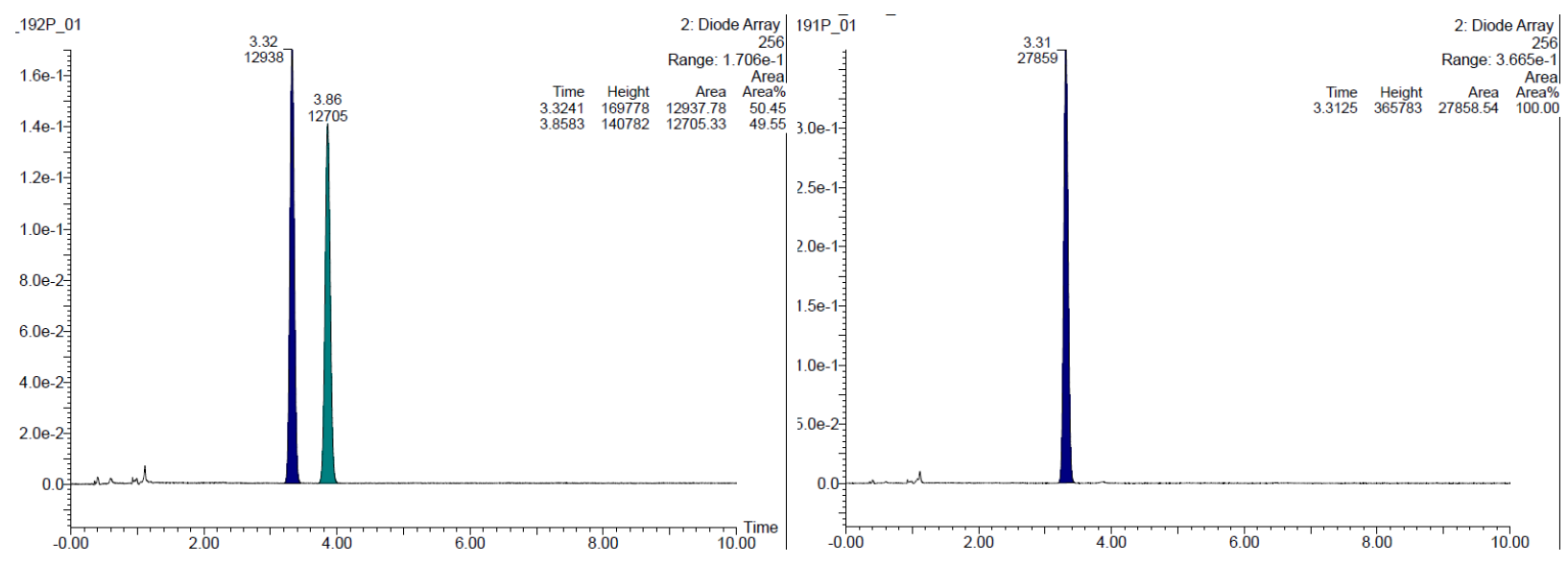<smiles>COc1ccc(CN2CCC[C@H](Nc3cccc(C(F)(F)F)c3)C2=O)cc1</smiles>

8b; Enantio-specificity determination: Thar SFC

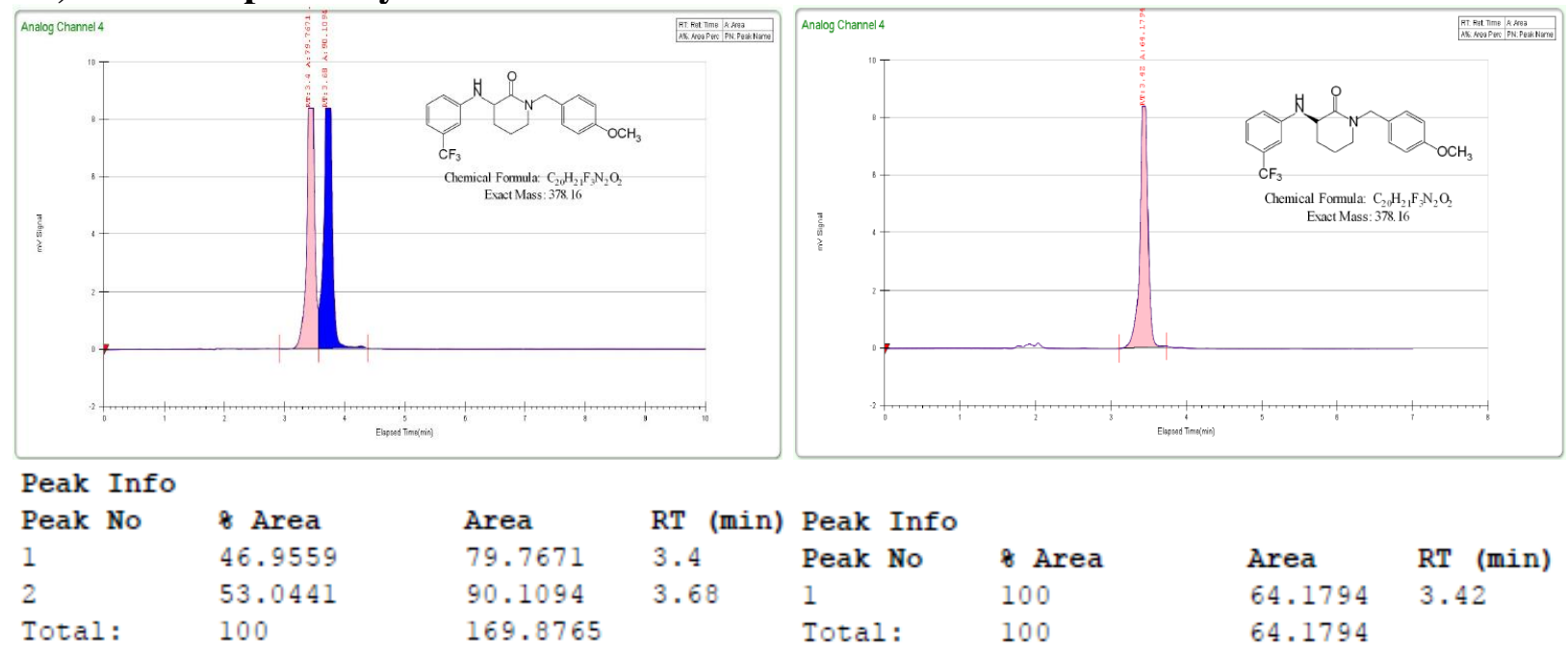


<smiles>COc1ccc(CN2CCC[C@H](Nc3cc(F)cc(C(F)(F)F)c3)C2=O)cc1</smiles>

8c; Enantio-specificity determination: Thar SFC

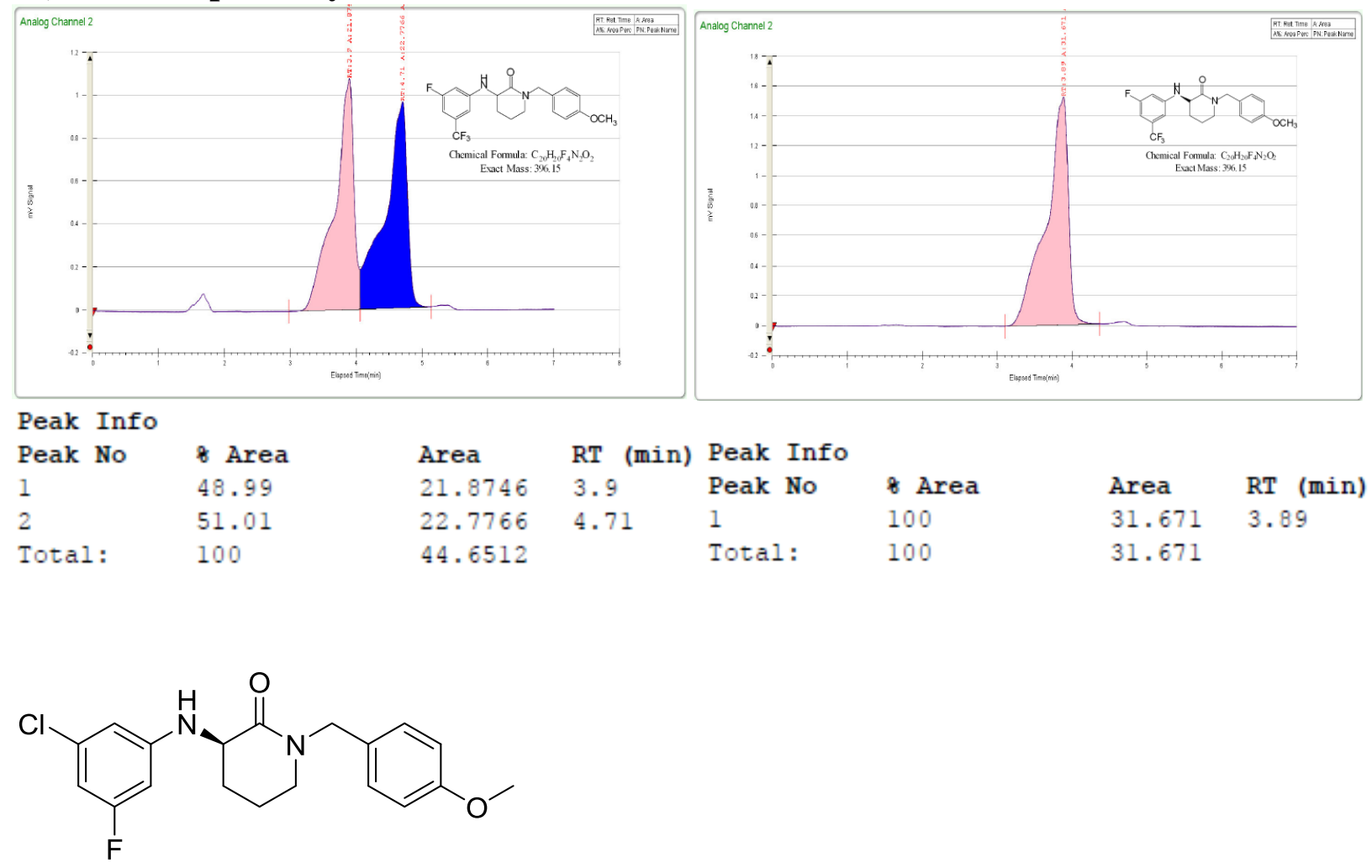

8d; Enantio-specificity determination: Thar SFC

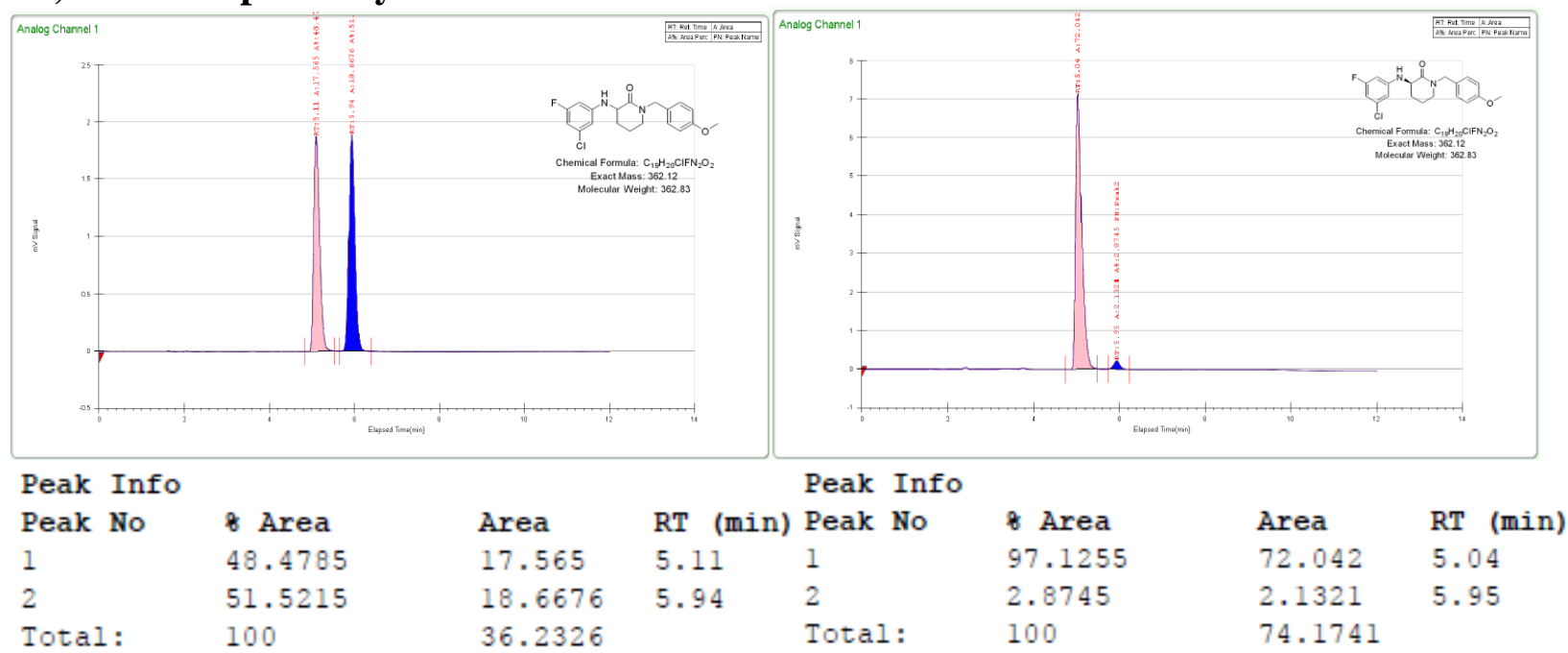


<smiles>COc1ccc(CN2CCC[C@H](Nc3ccncc3)C2=O)cc1</smiles>

8e; Enantio-specificity determination: Thar SFC
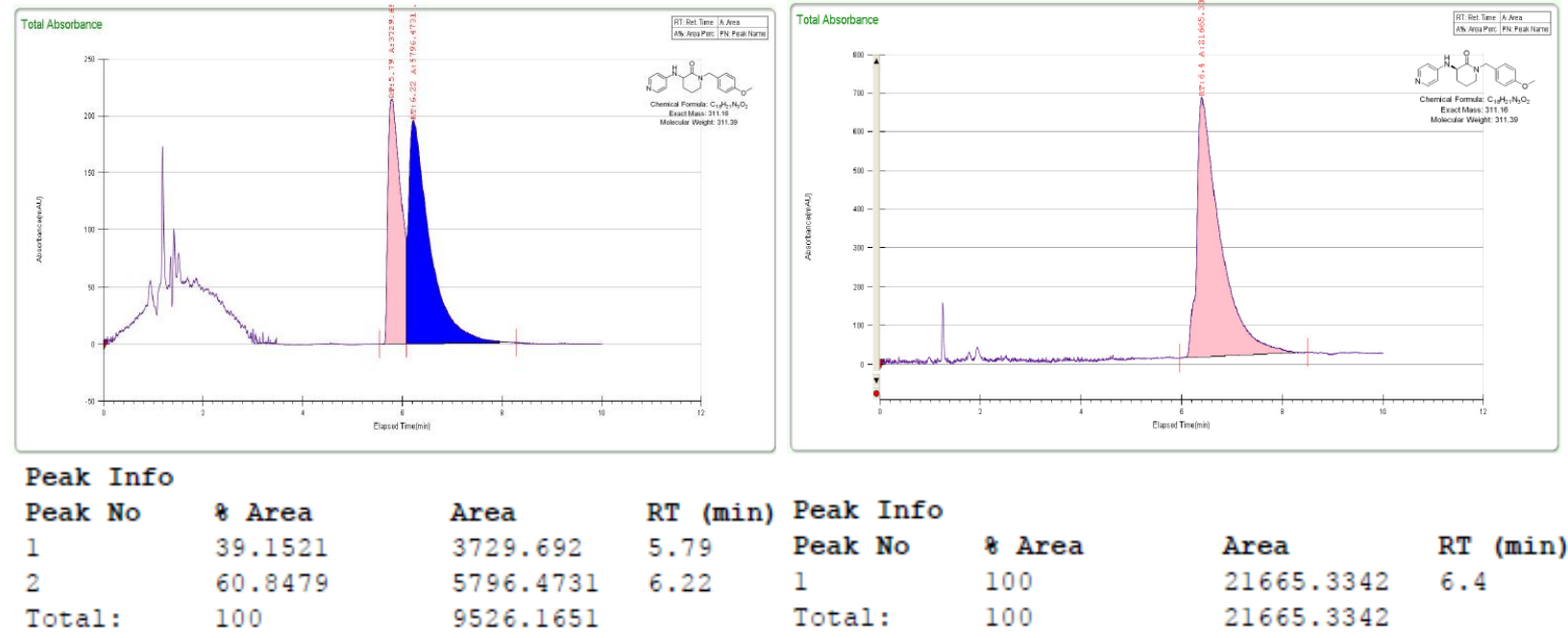<smiles>COc1ccc(CN2CCC[C@H](Nc3cccnc3)C2=O)cc1</smiles>

8f; Enantio-specificity determination: Thar SFC

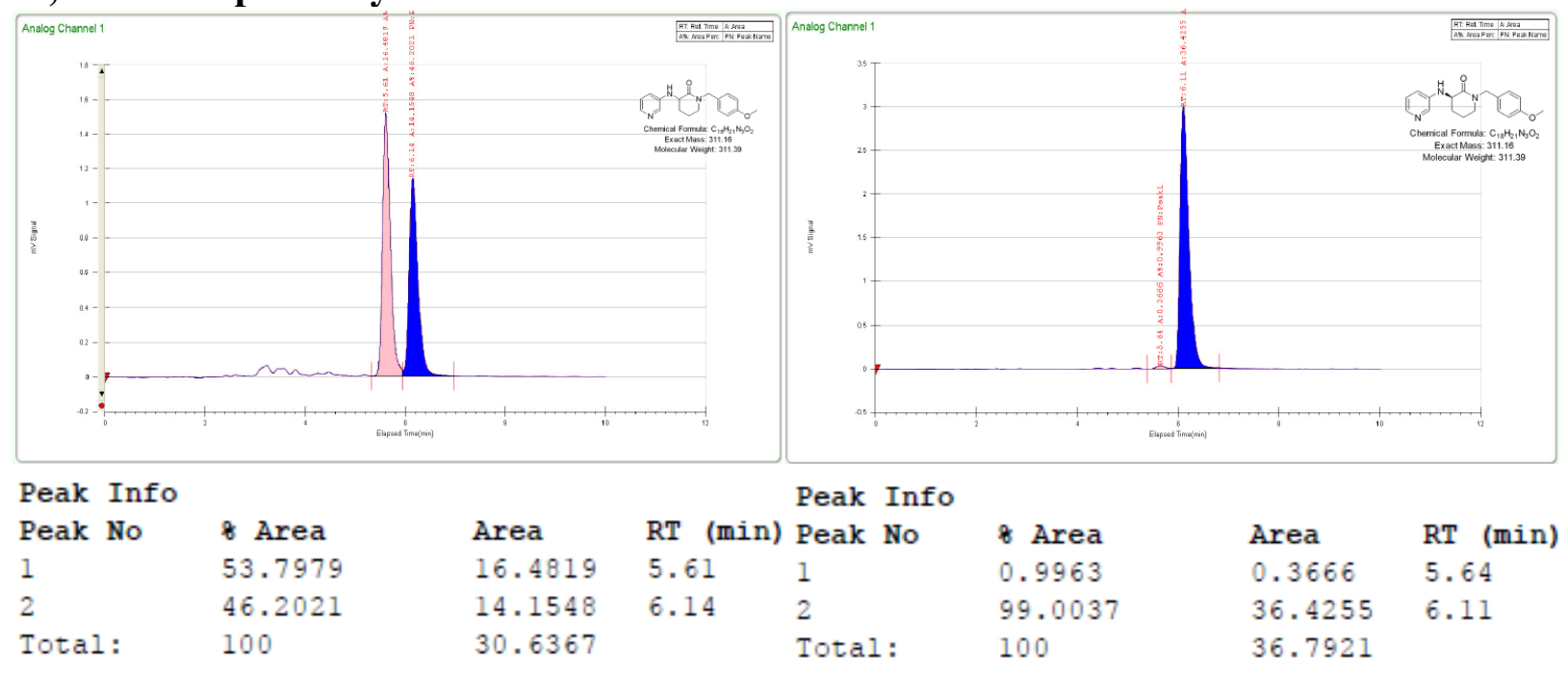


<smiles>COc1ccc(CN2CCC[C@H](Nc3ccccn3)C2=O)cc1</smiles>

8g; Enantio-specificity determination: Thar SFC

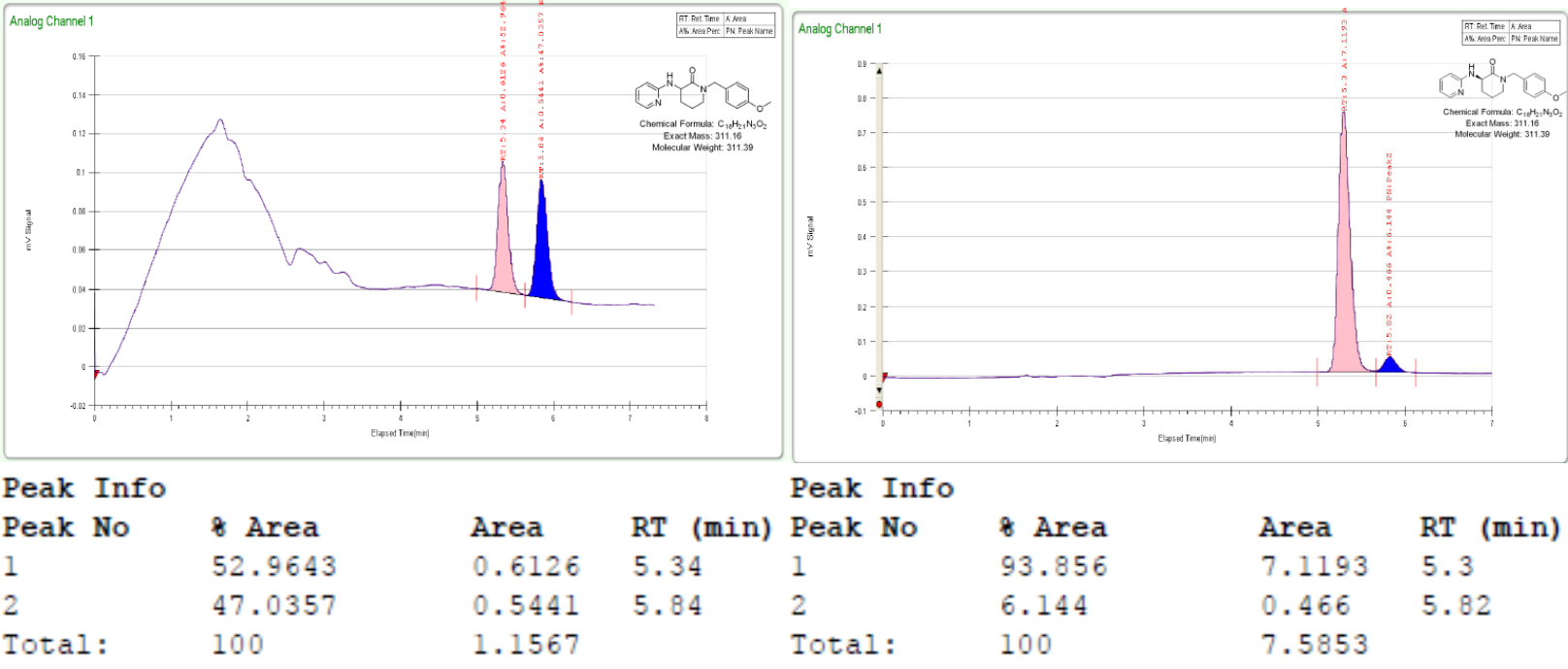<smiles>COc1ccc(CN2CCC[C@H](Nc3ccccc3)C2=O)cc1</smiles>

8h; Enantio-specificity determination: Thar SFC

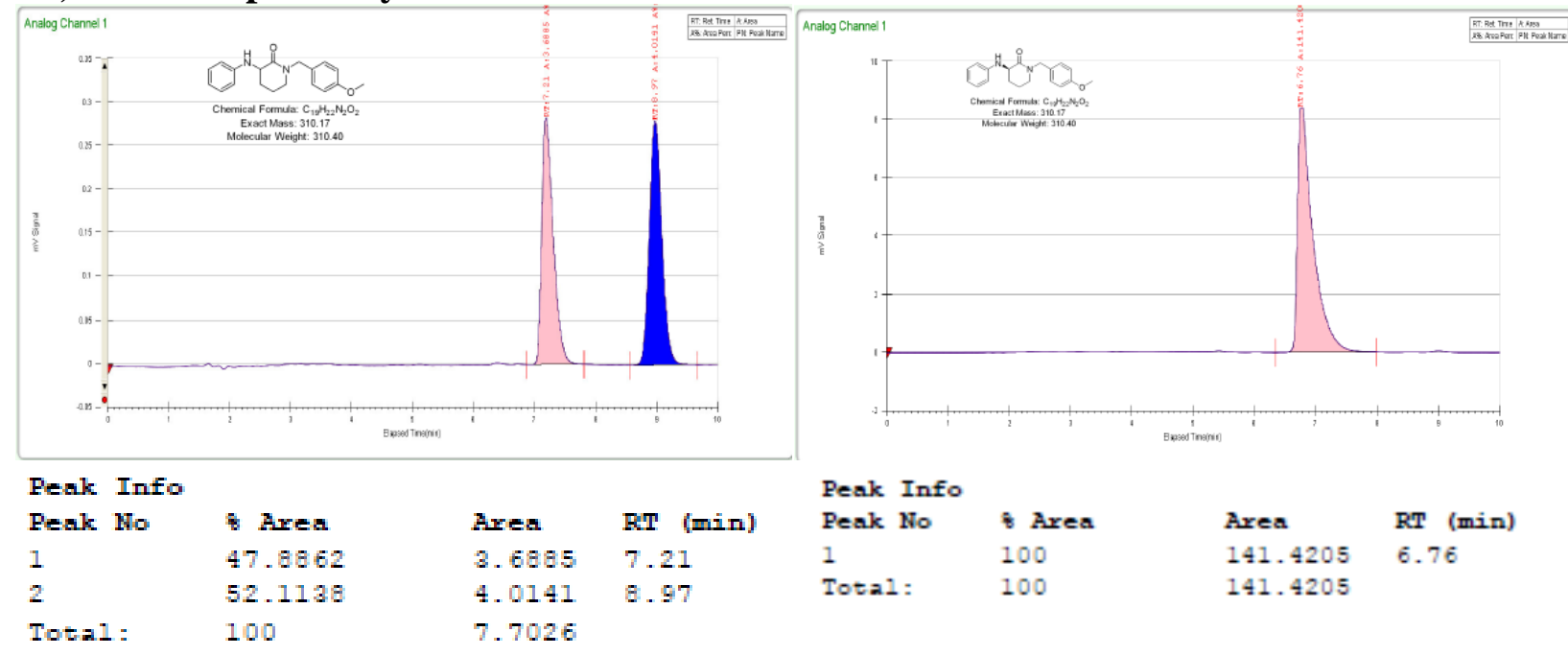


<smiles>COc1ccc(CN2CCC[C@H](Nc3cccc(OC)c3)C2=O)cc1</smiles>

8i; Enantio-specificity determination: Thar SFC

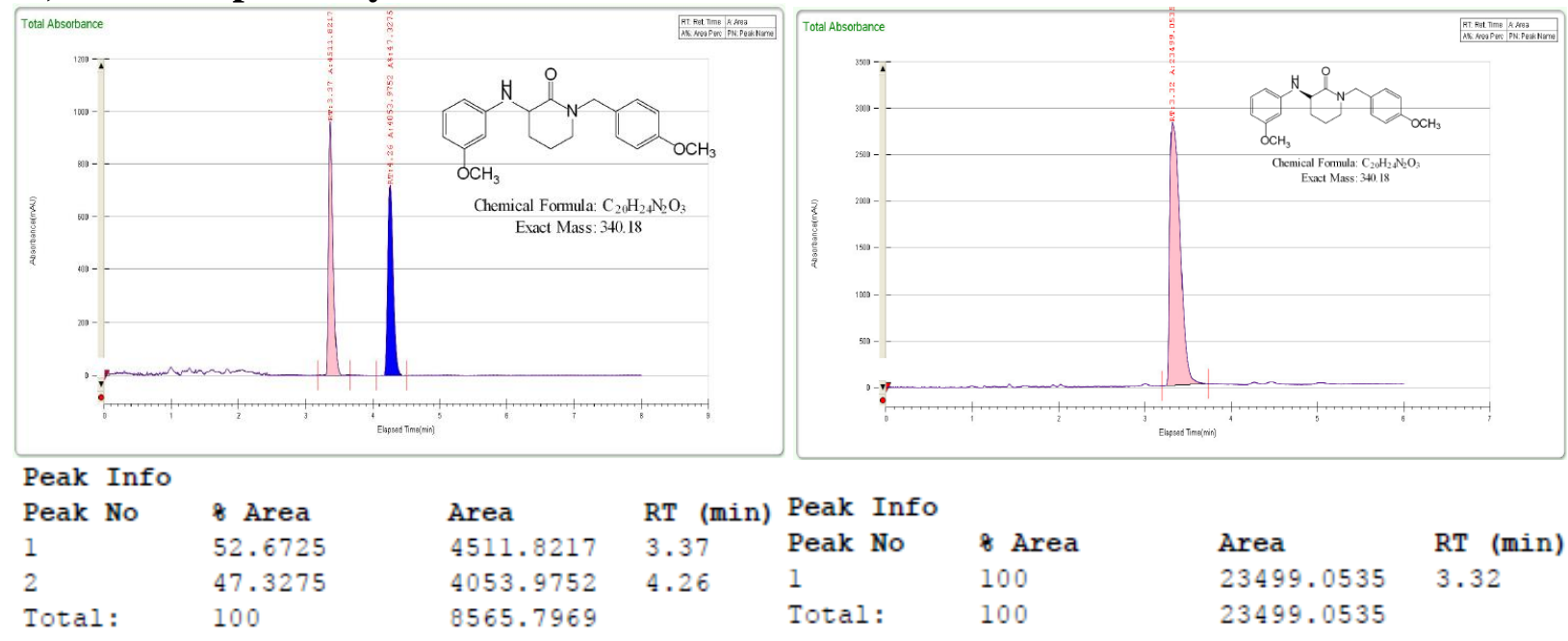<smiles>COc1ccc(CN2CCC[C@H](Nc3ccccc3OC)C2=O)cc1</smiles>

8j; Enantio-specificity determination: Thar SFC
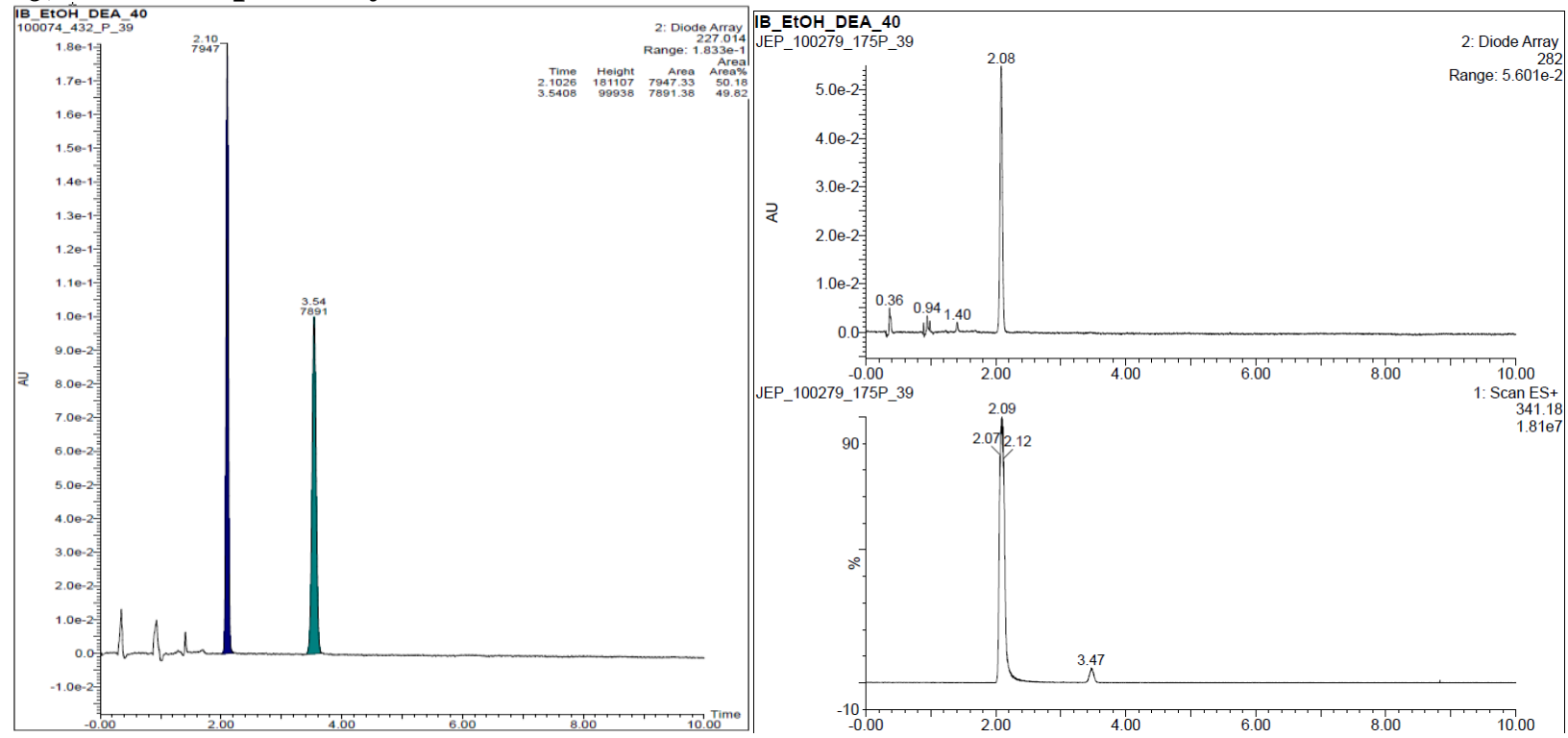
Page S11<smiles>O=C1CCCN1c1ccc(C(F)(F)F)cc1</smiles>

1a

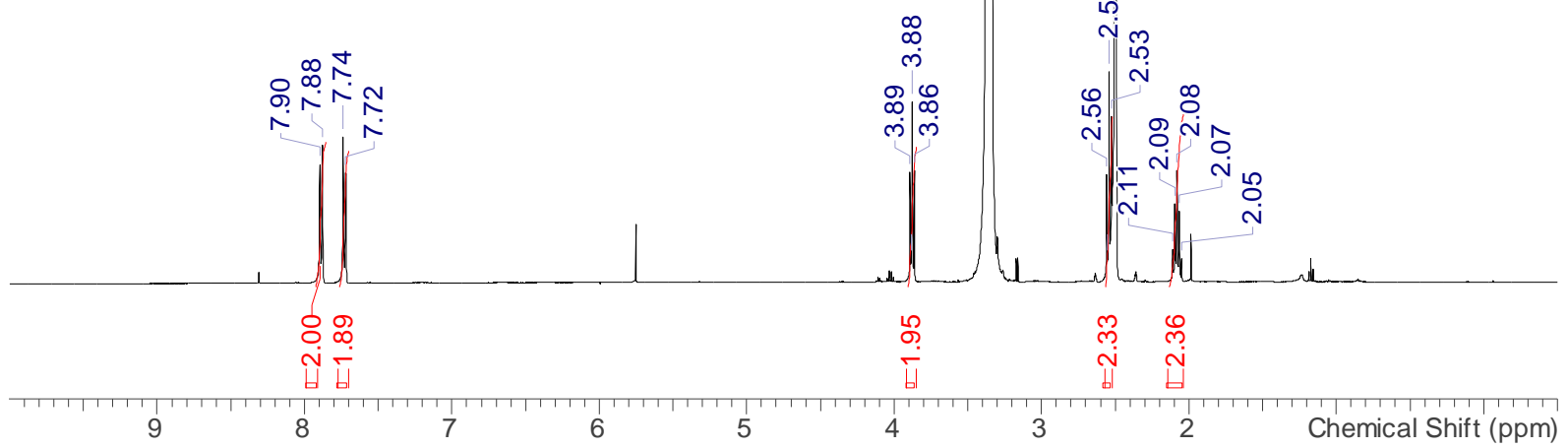<smiles>O=C1CCCN1c1ccncc1</smiles>

1b

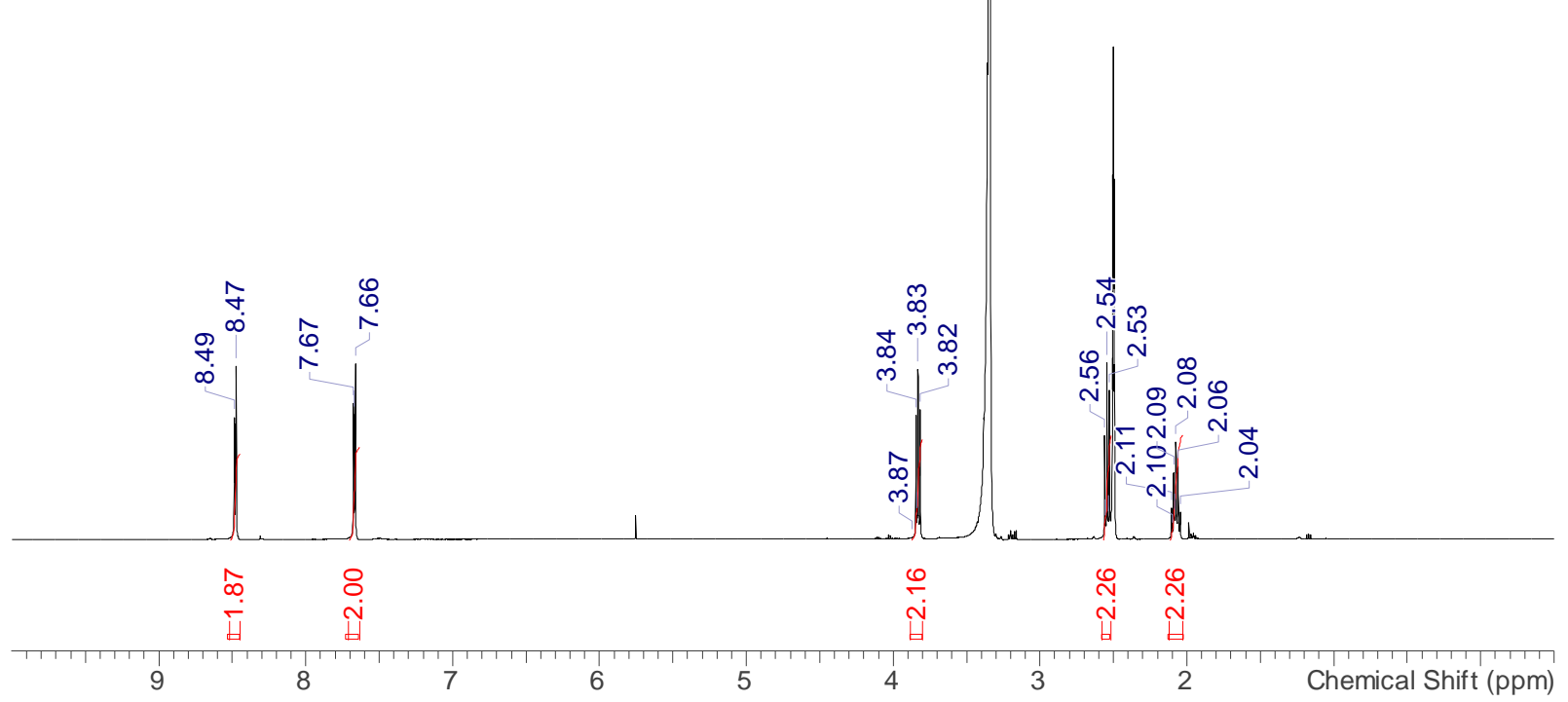


Page S12
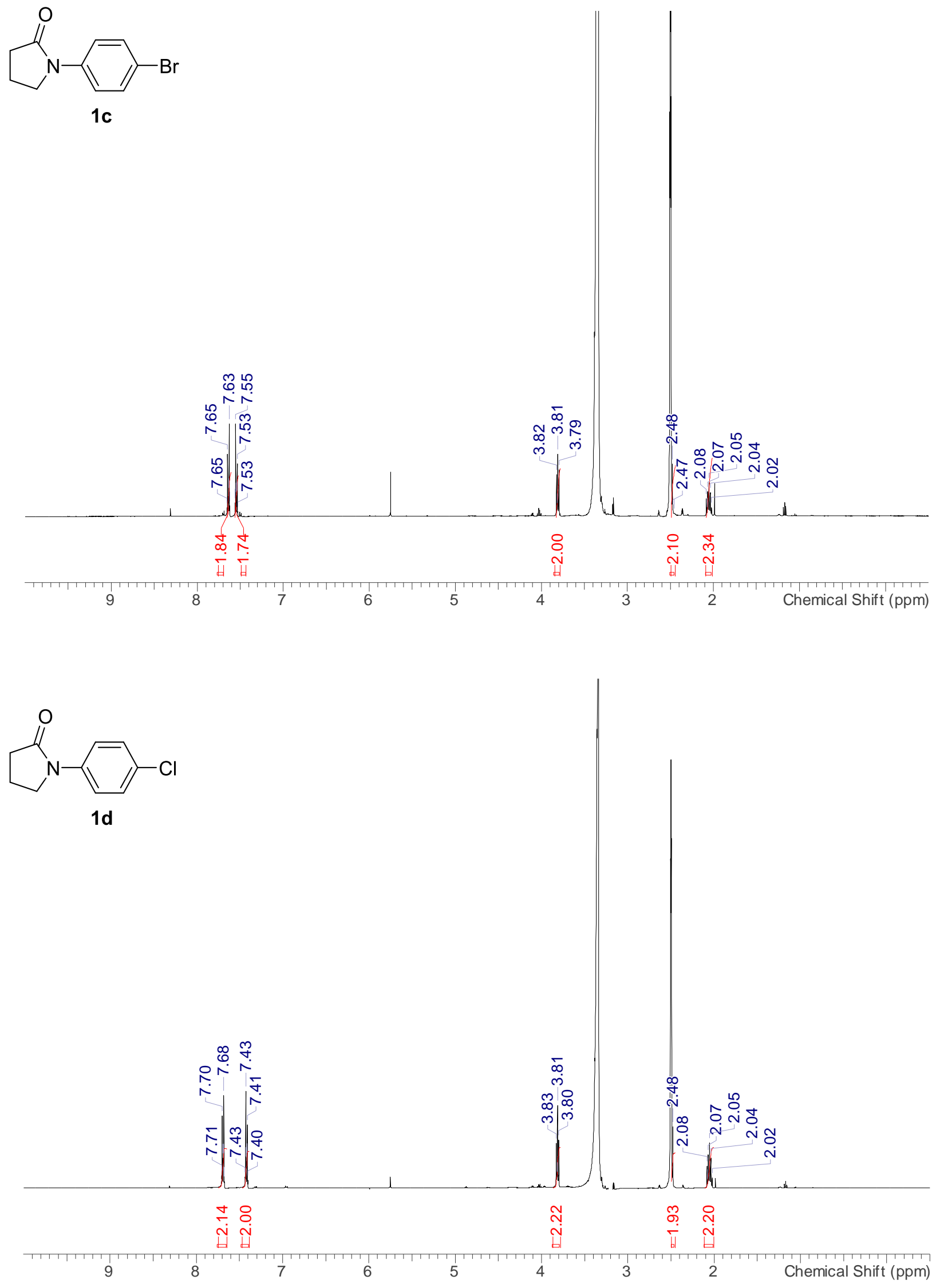
Page S13
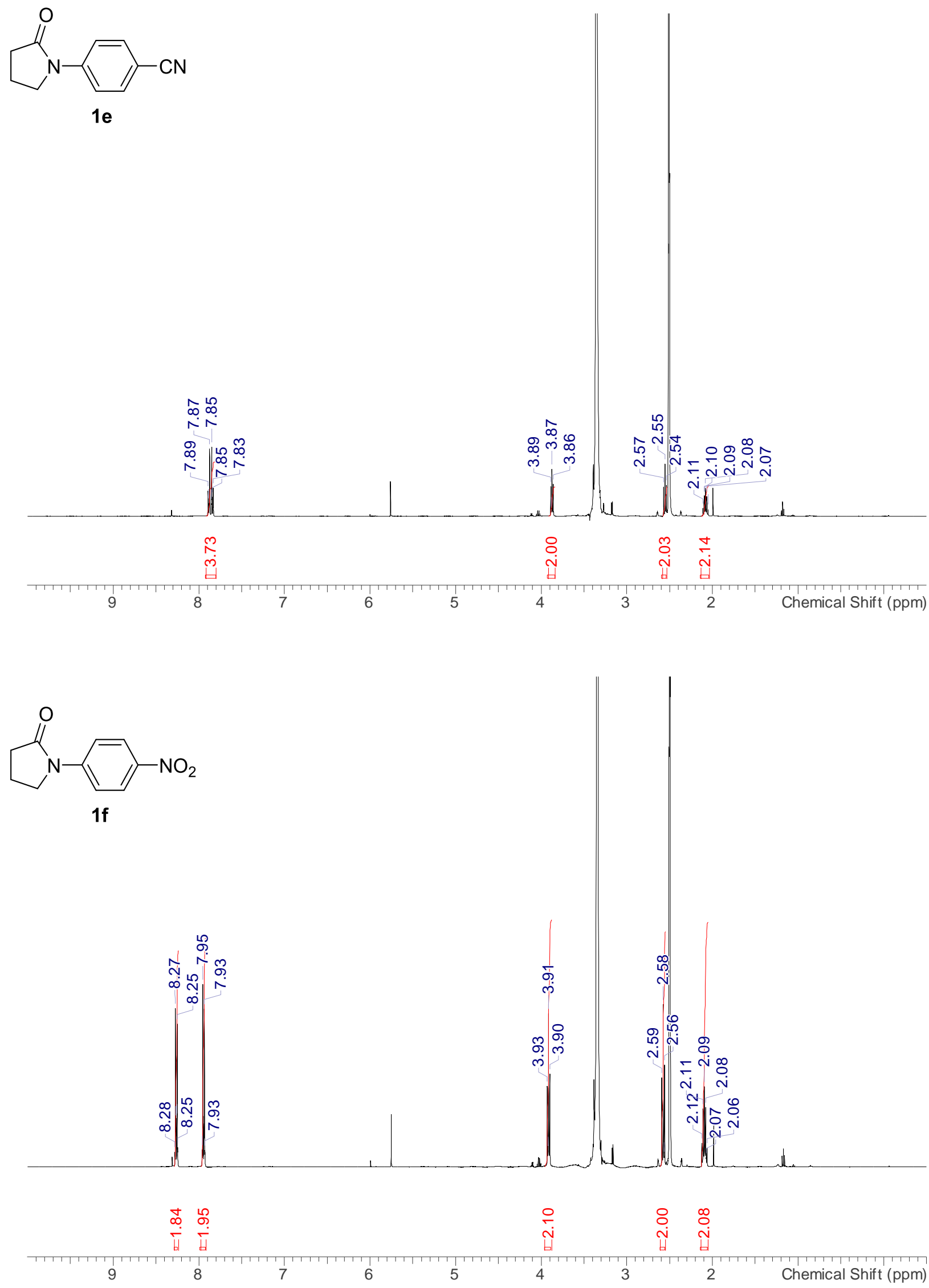
Page S14<smiles>CCOC(=O)c1ccc(N2CCCC2=O)cc1</smiles>
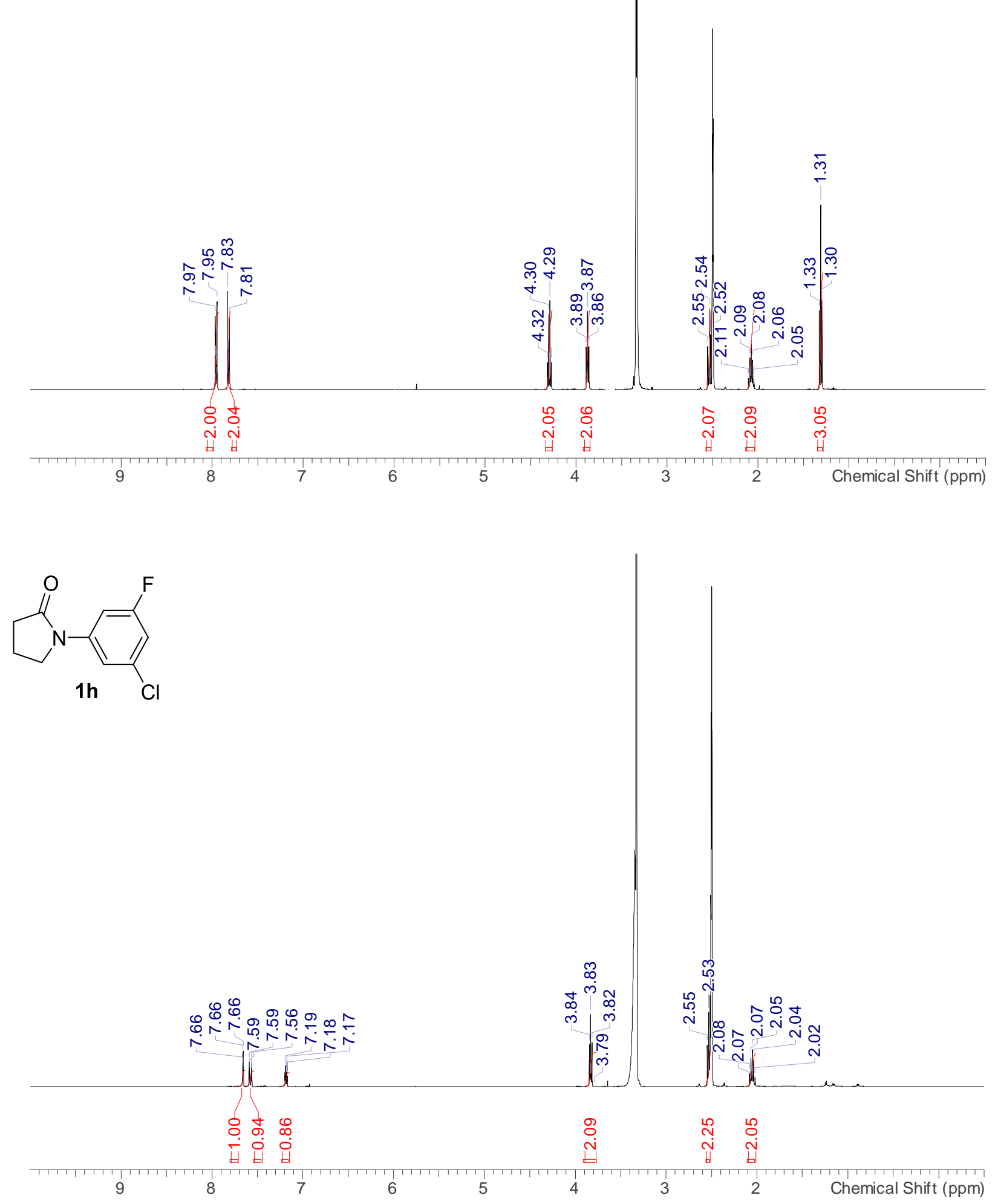
Page S15
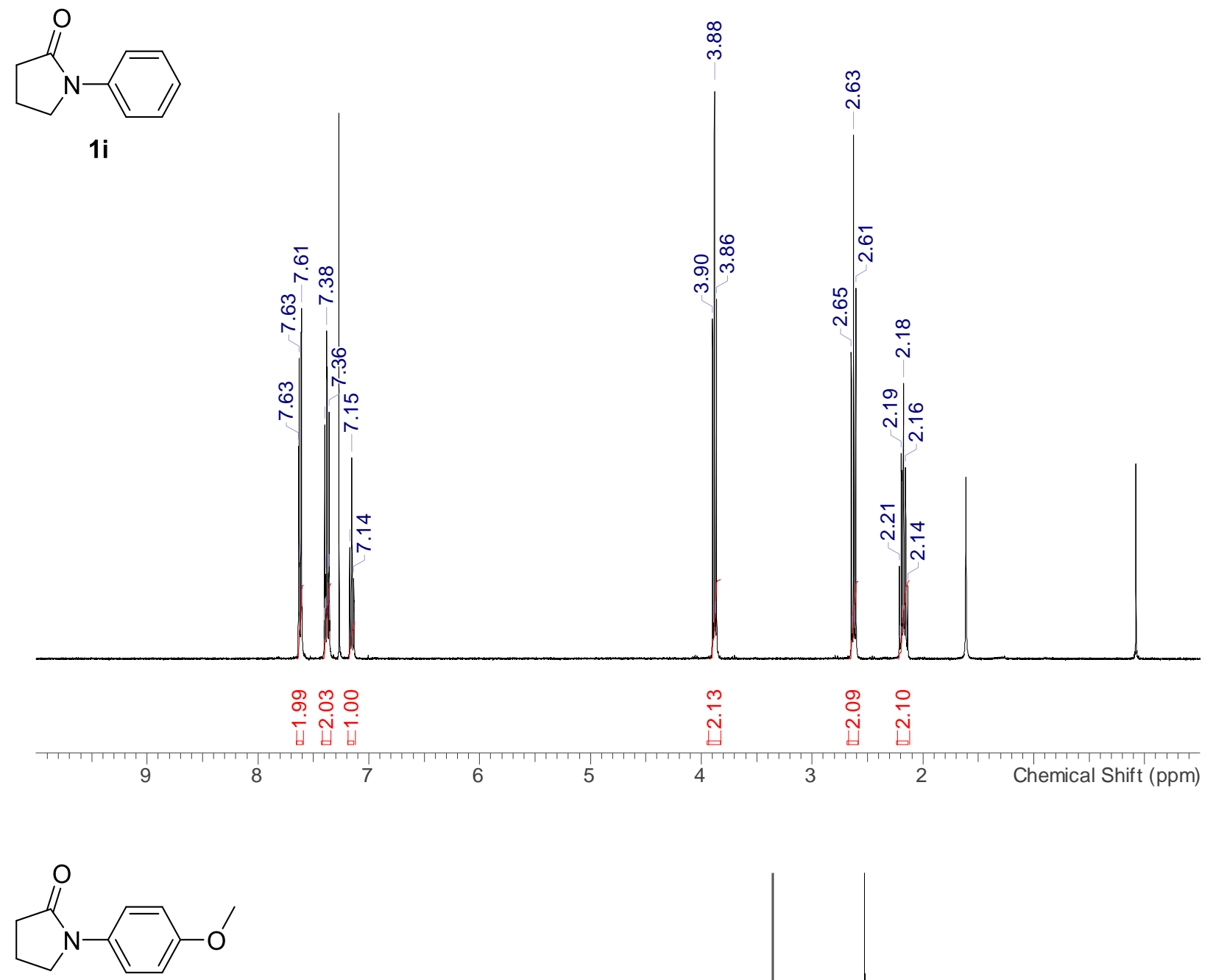

1j

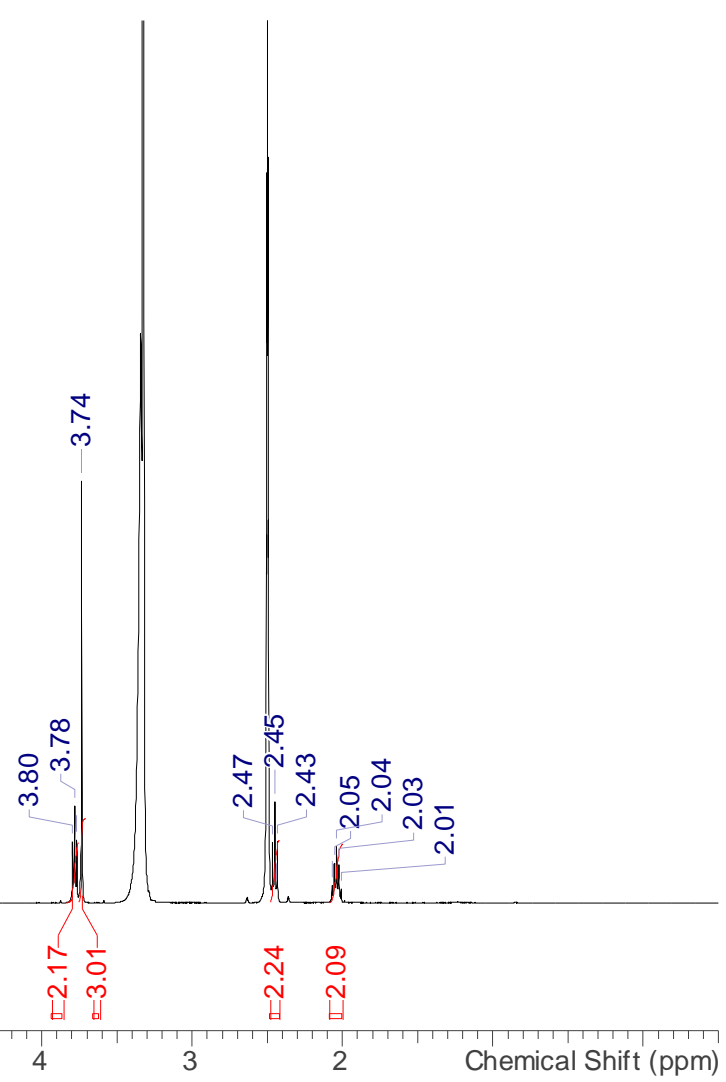


Page S16

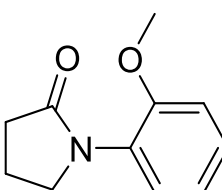

$1 k$ $\forall$
$\dot{\infty}$
$\dot{m}$
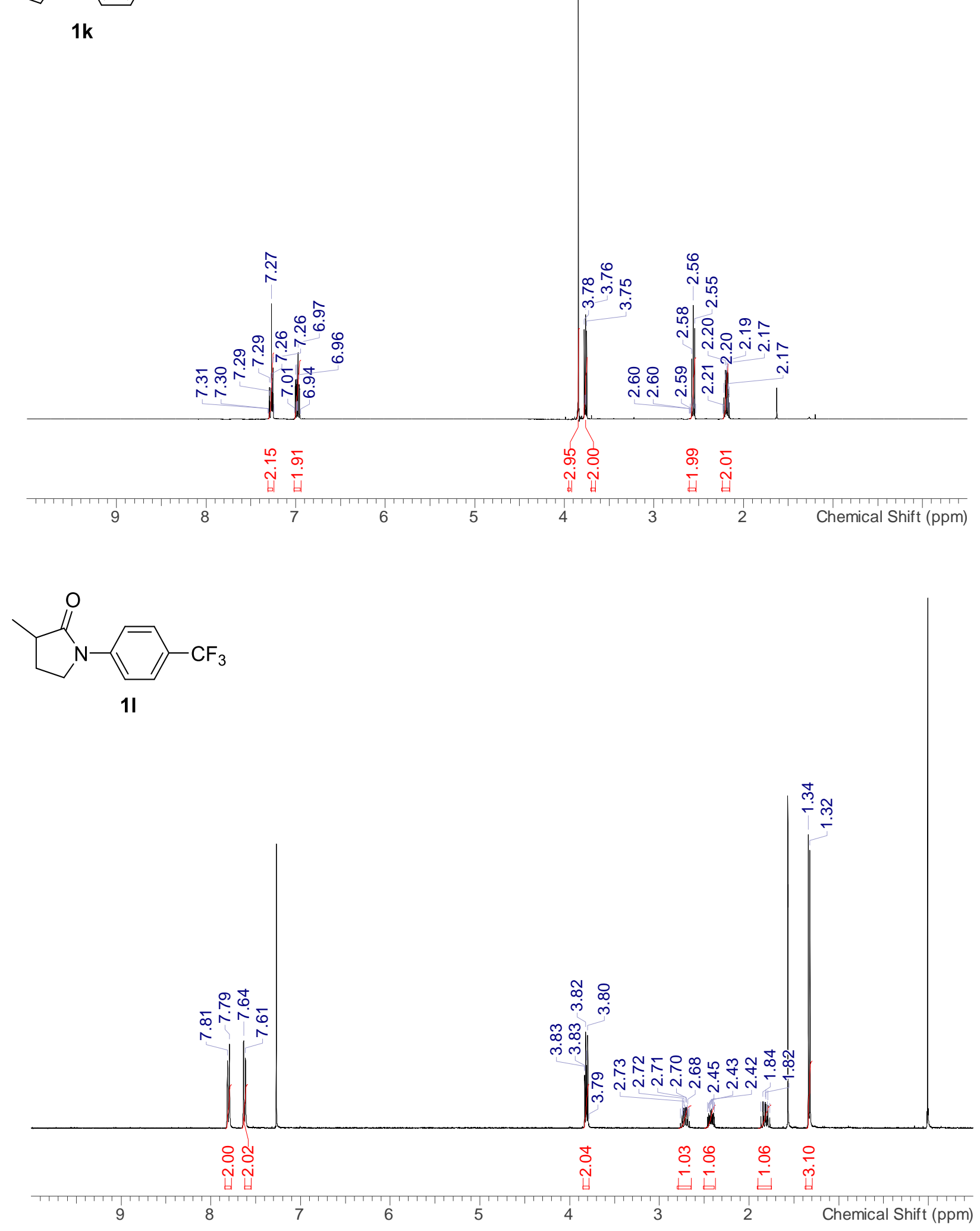
Page S17
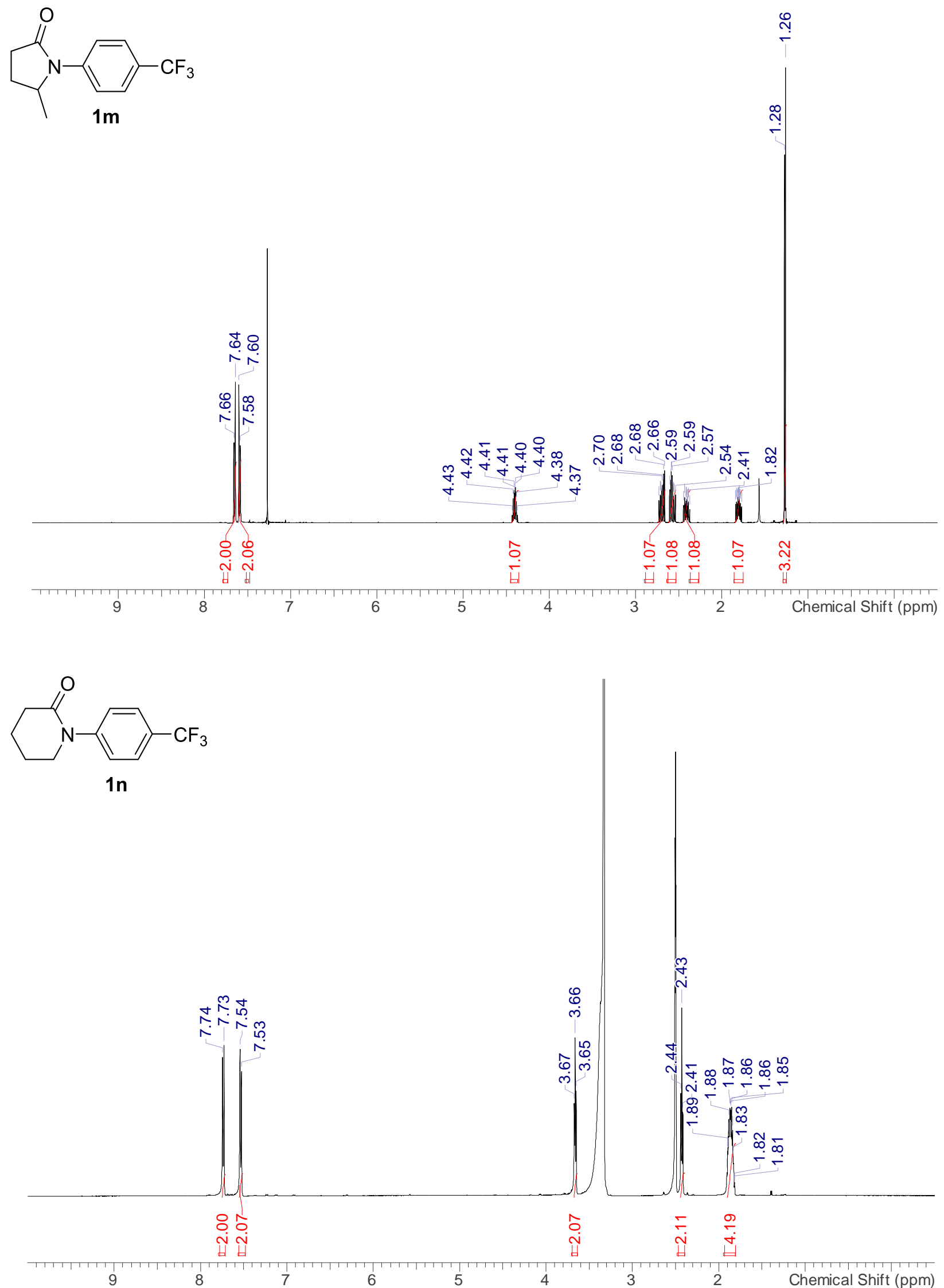
Page S18
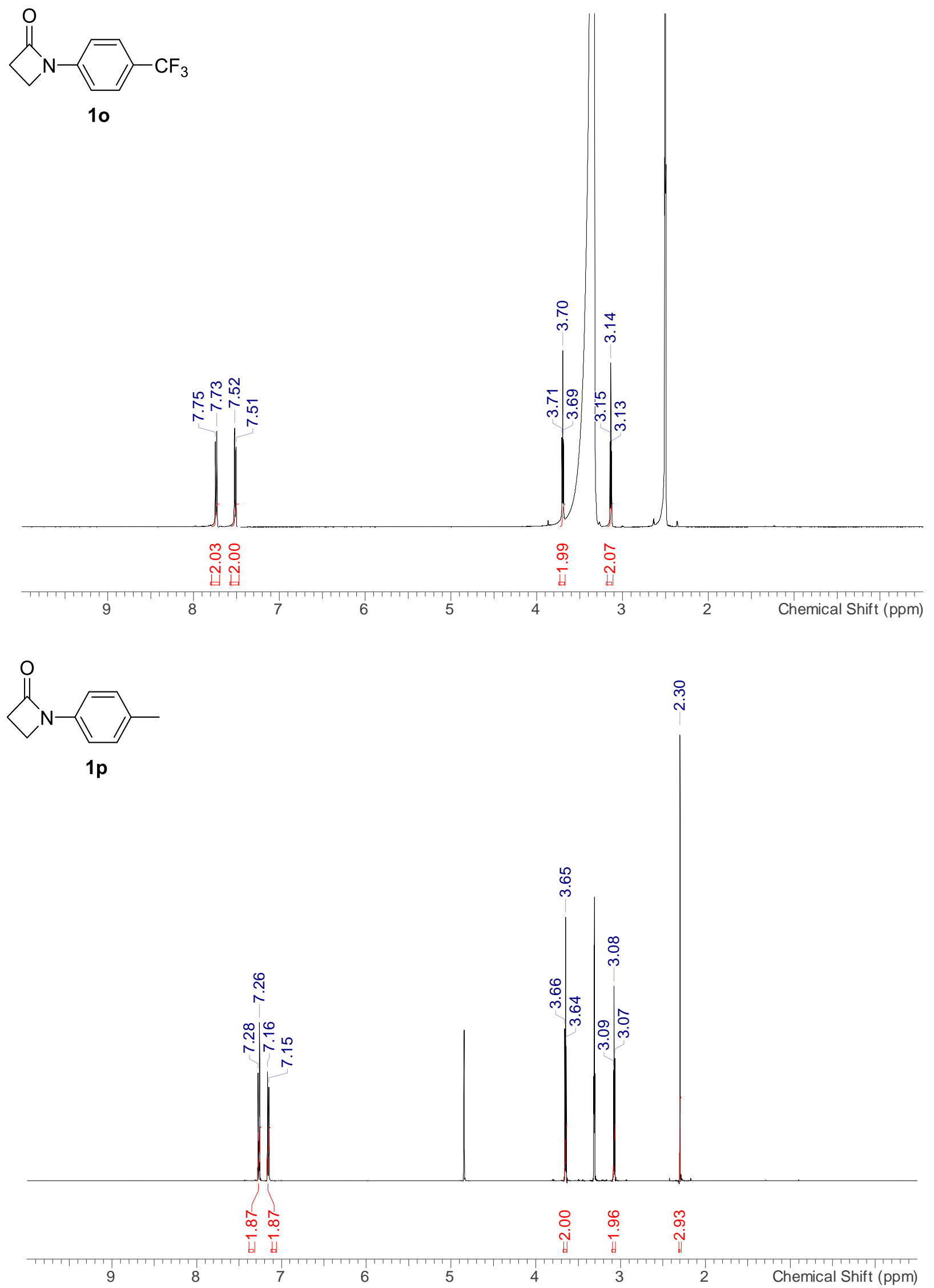
Page S19

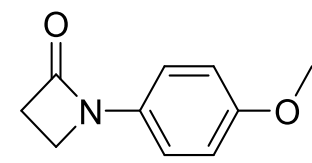

$\hat{N}$

1q

छे

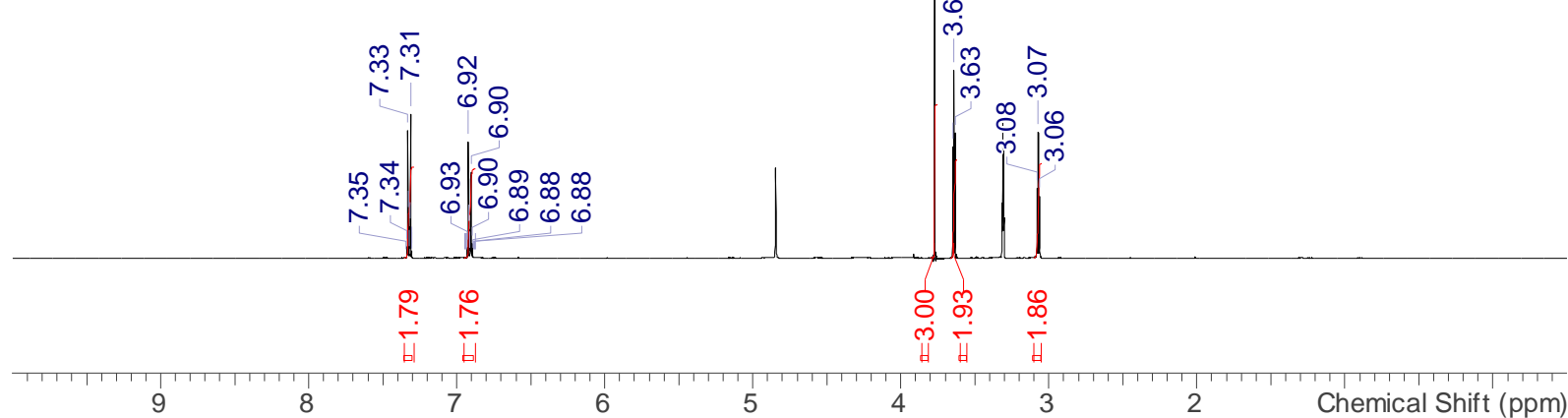

$\underbrace{\mathrm{N}}_{1 \mathrm{r}} \mathrm{O}^{\mathrm{CF}}$

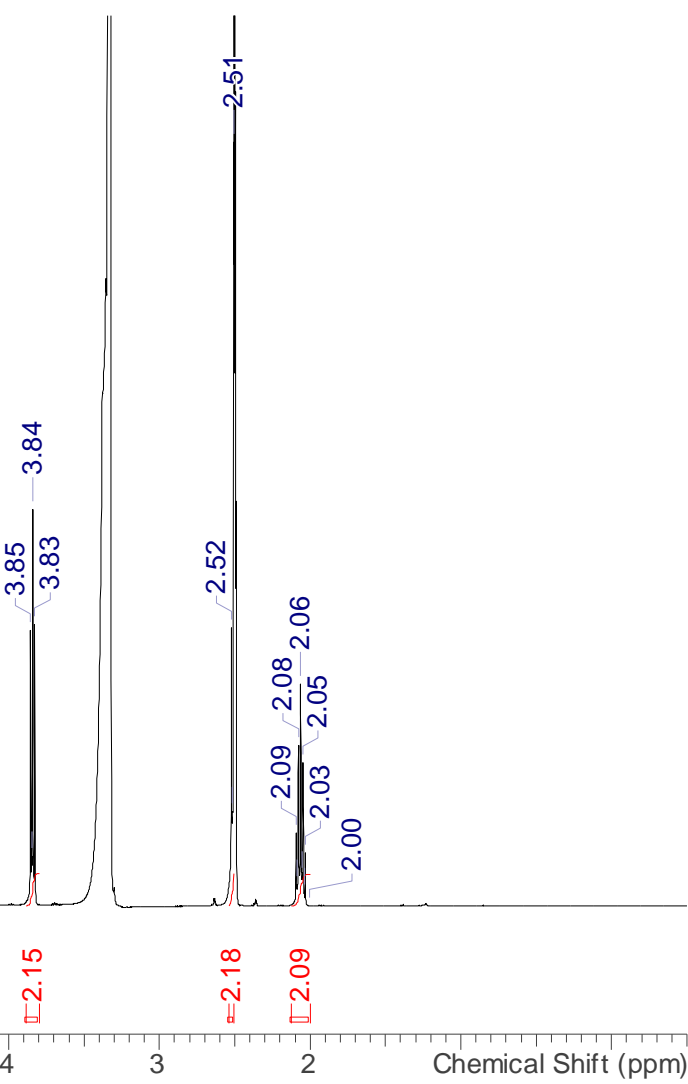


Page S20<smiles>COc1ccc(CNCCCCNc2ccc(C(F)(F)F)cc2)cc1</smiles>

3a
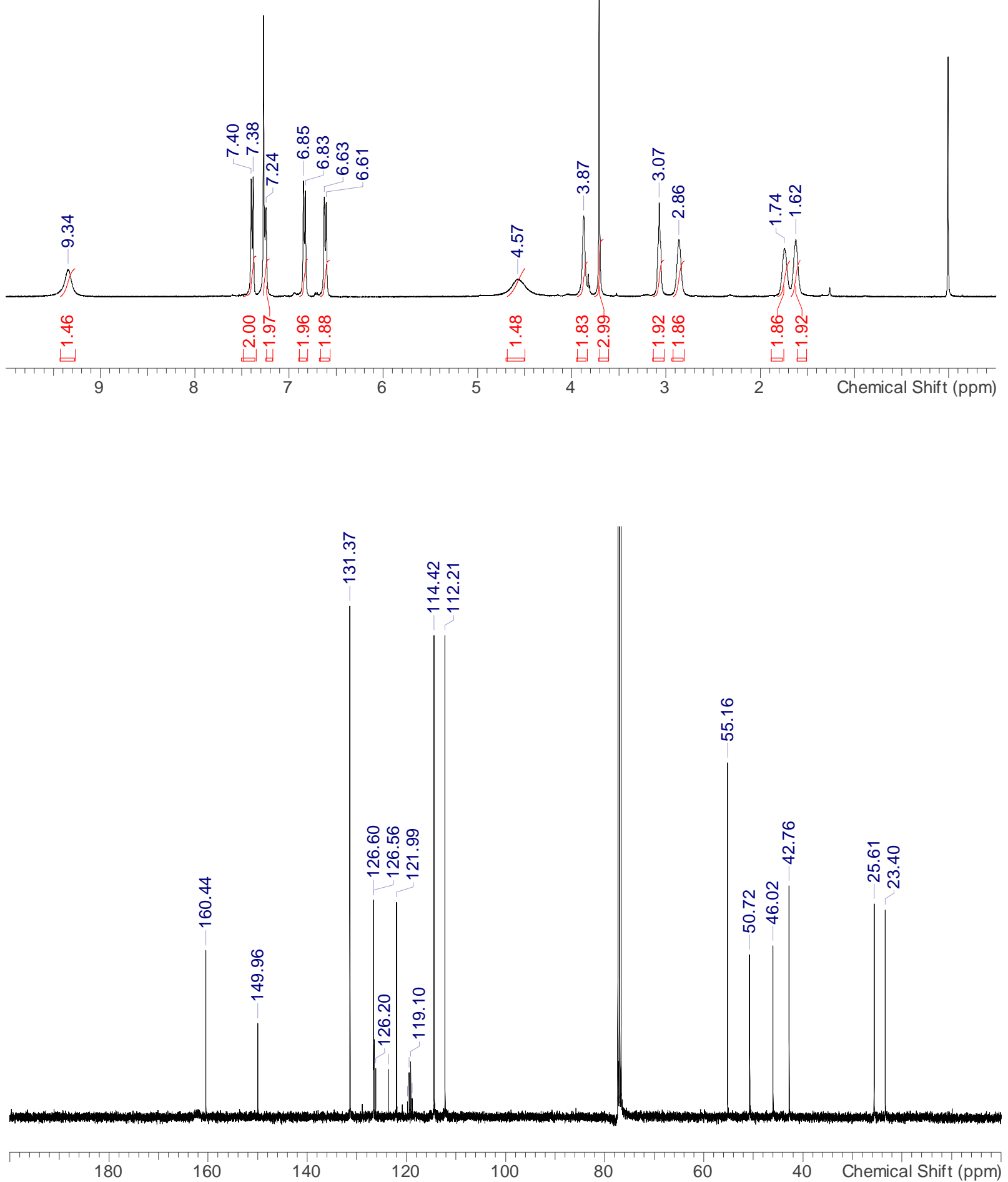
Page S21<smiles>COc1ccc(CNCCCCNc2ccncc2)cc1</smiles>

$3 b$
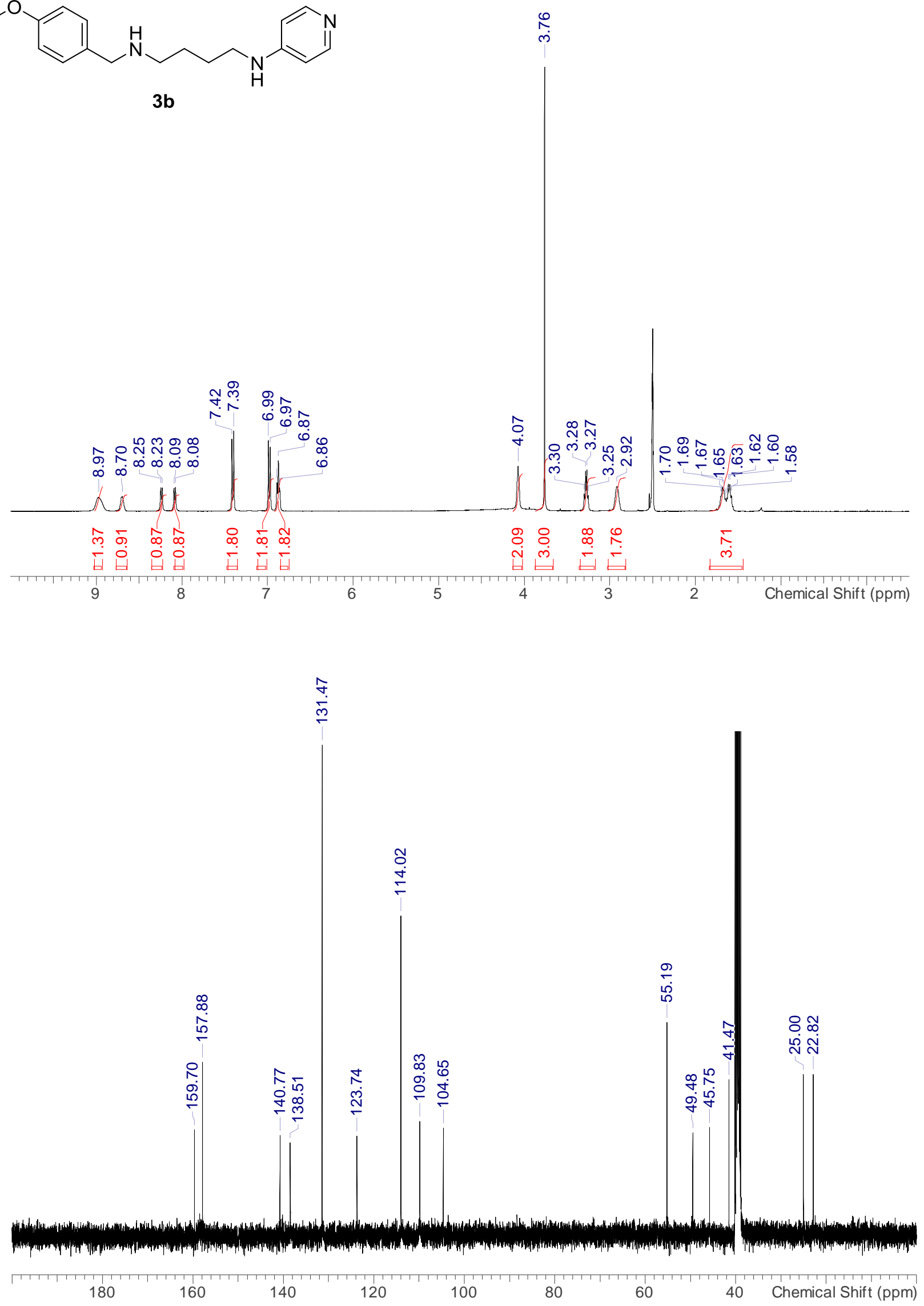
Page S22
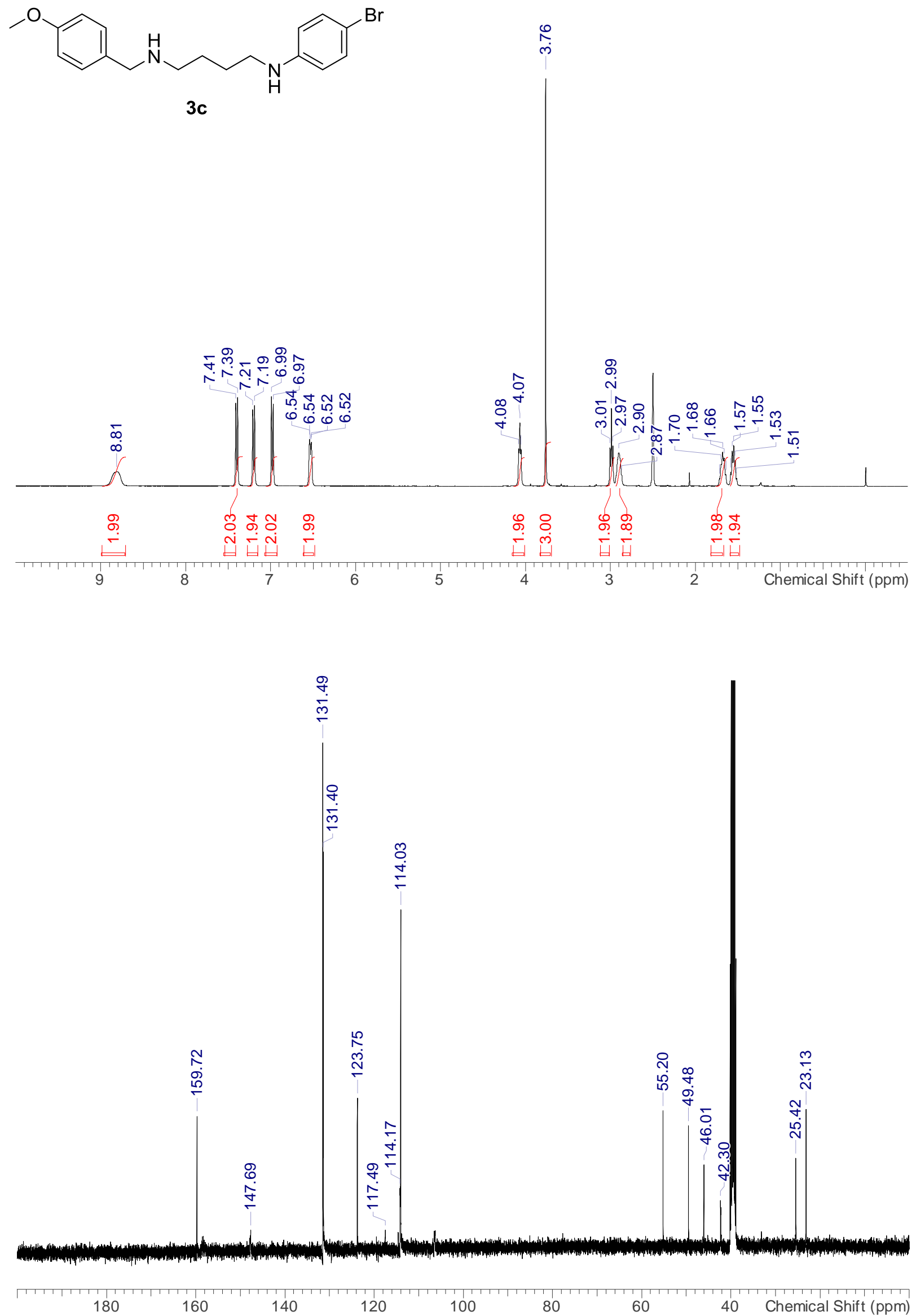
Page S23
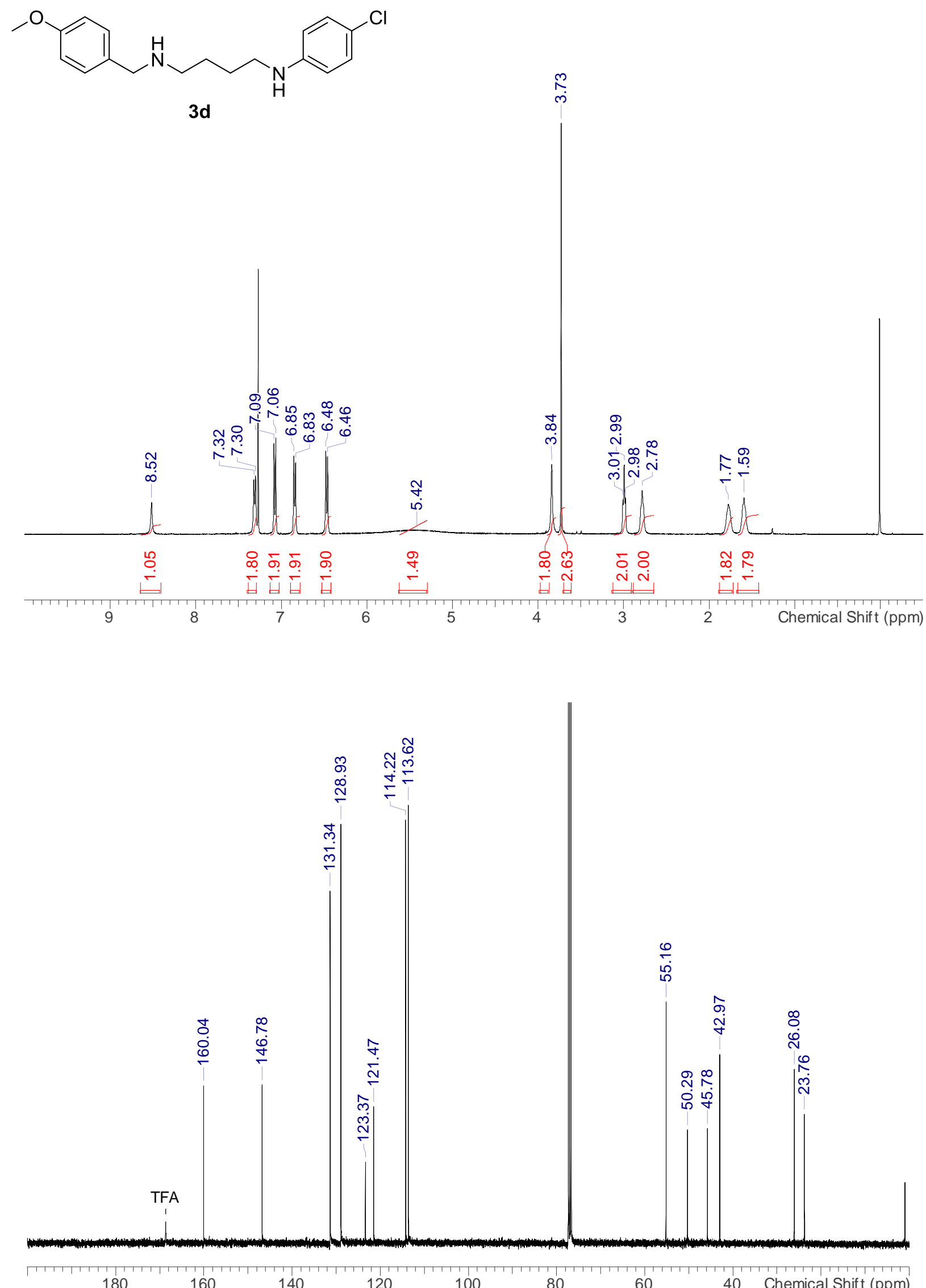
<smiles>COc1ccc(CNCCCCNc2ccc(C#N)cc2)cc1</smiles>

$3 e$
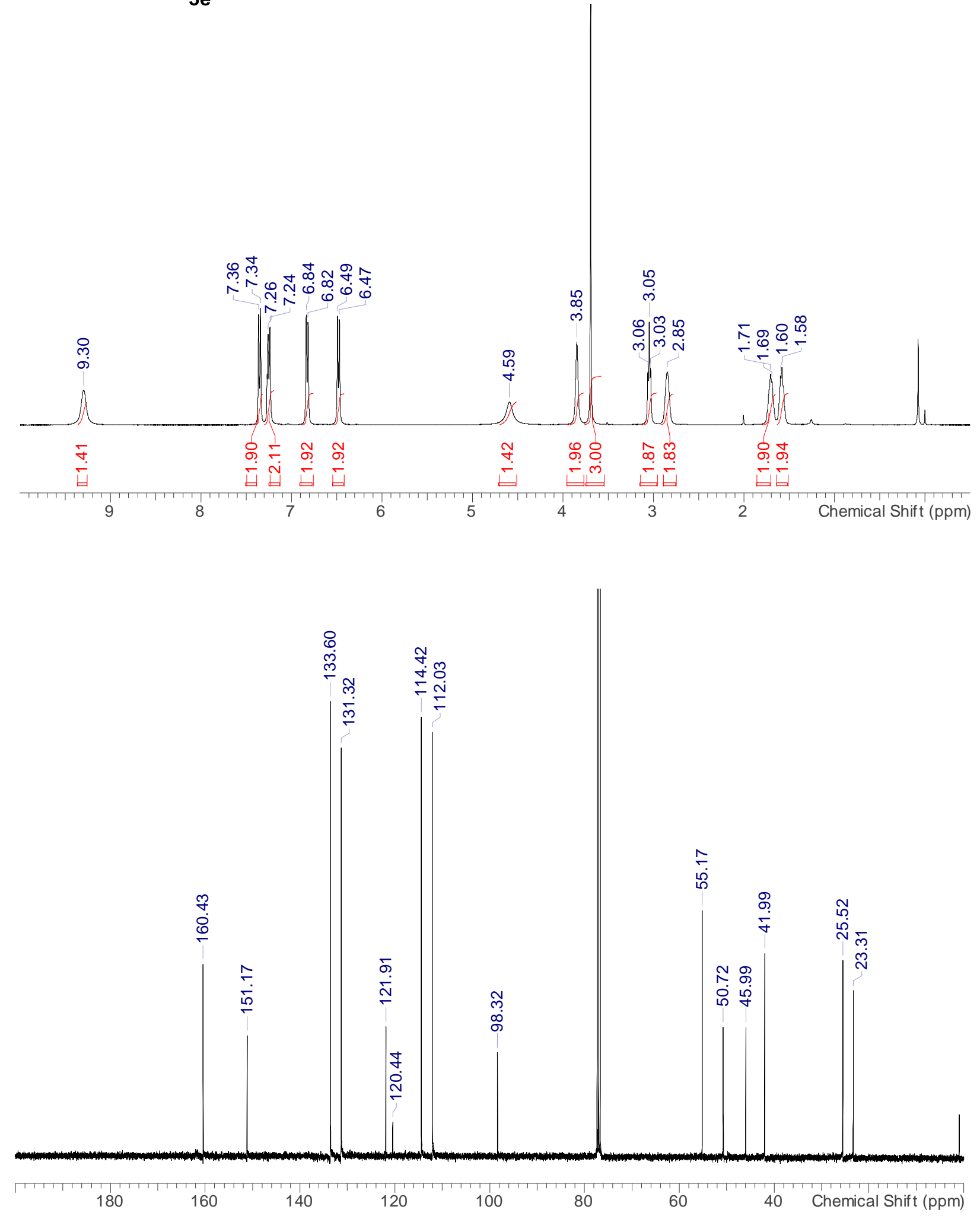
Page S25
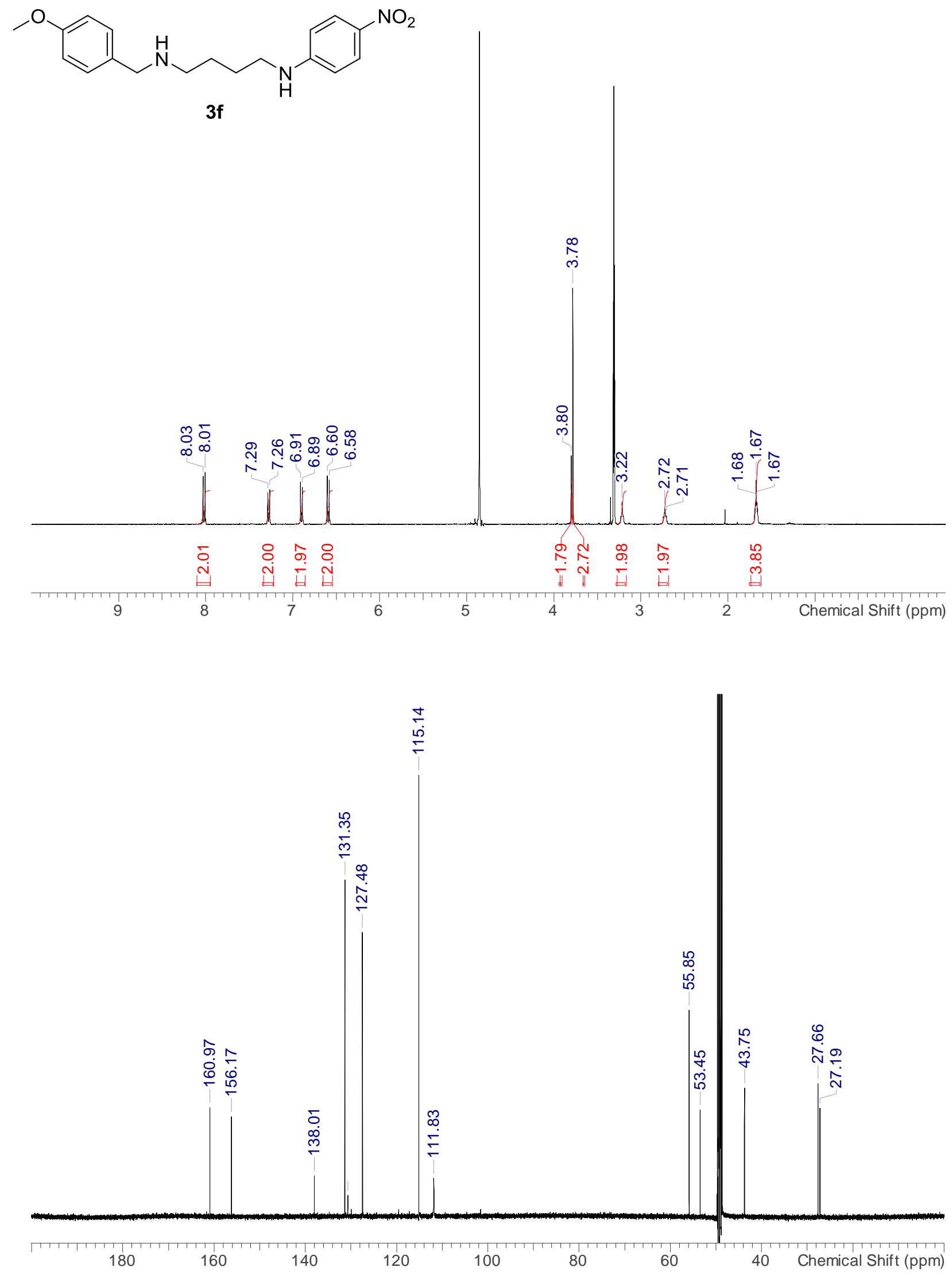

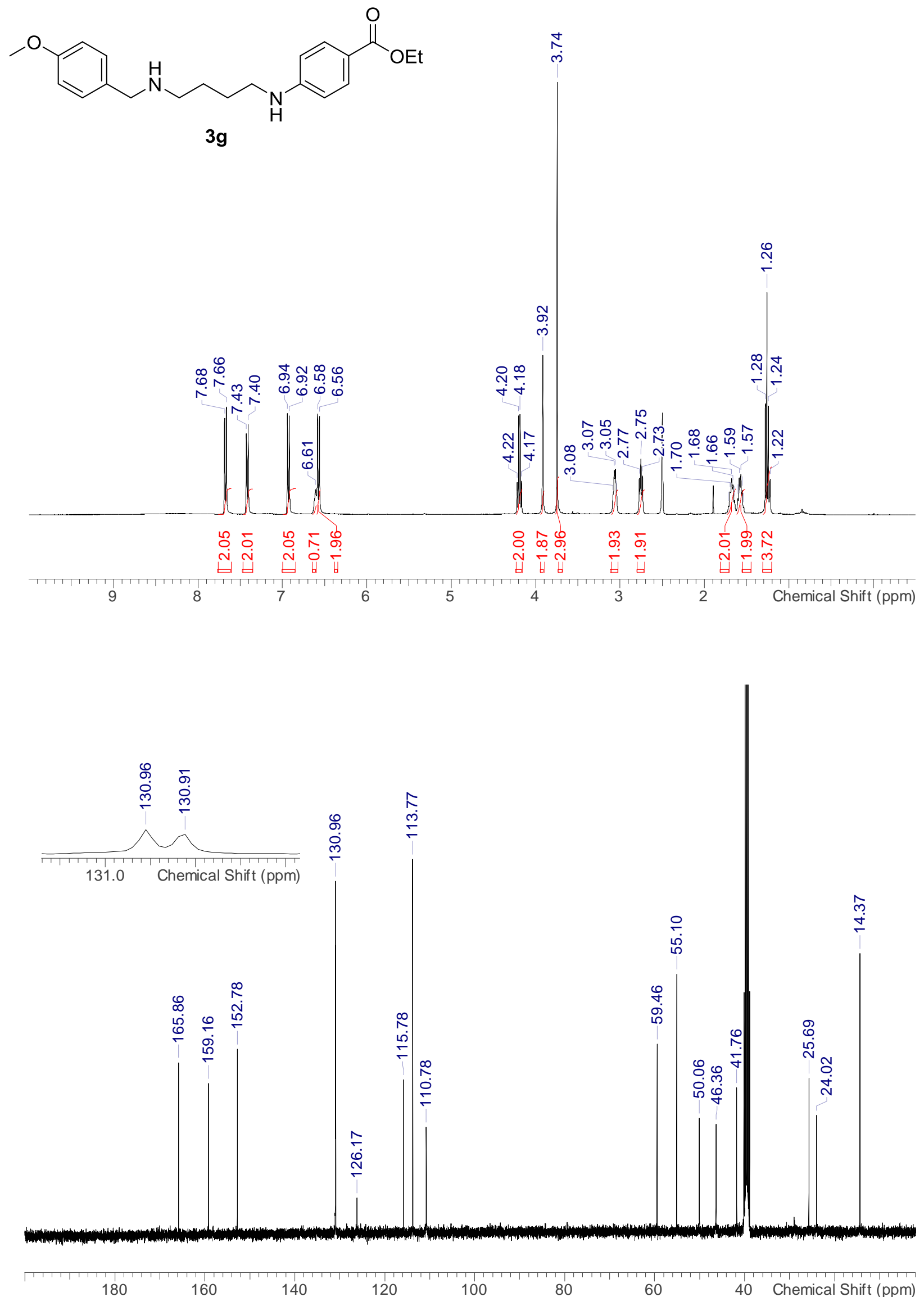
Page S27

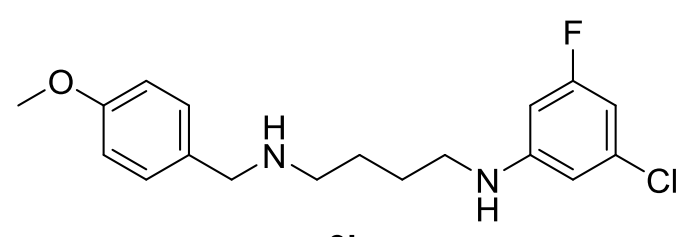

$3 h$
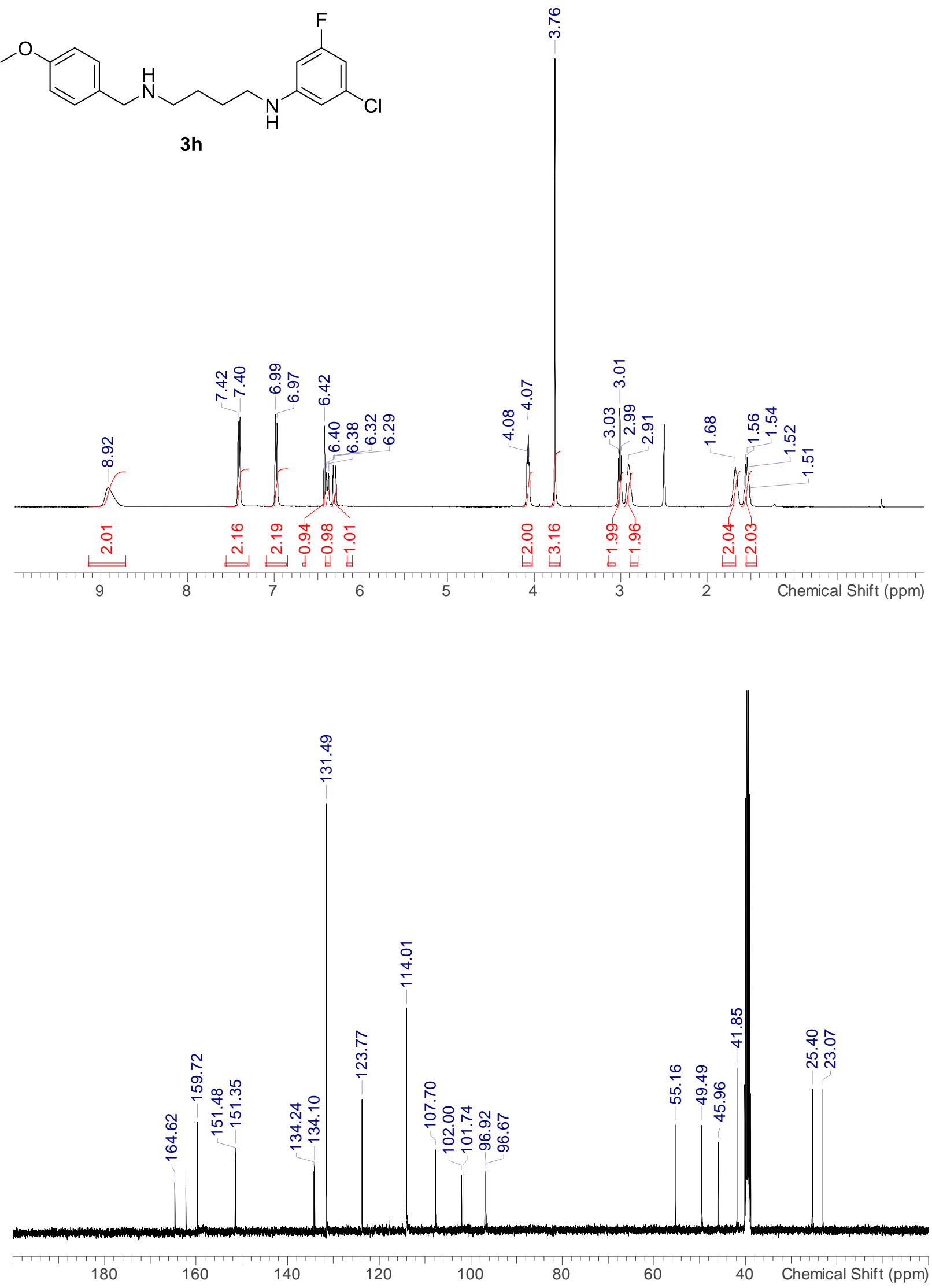
Page S28
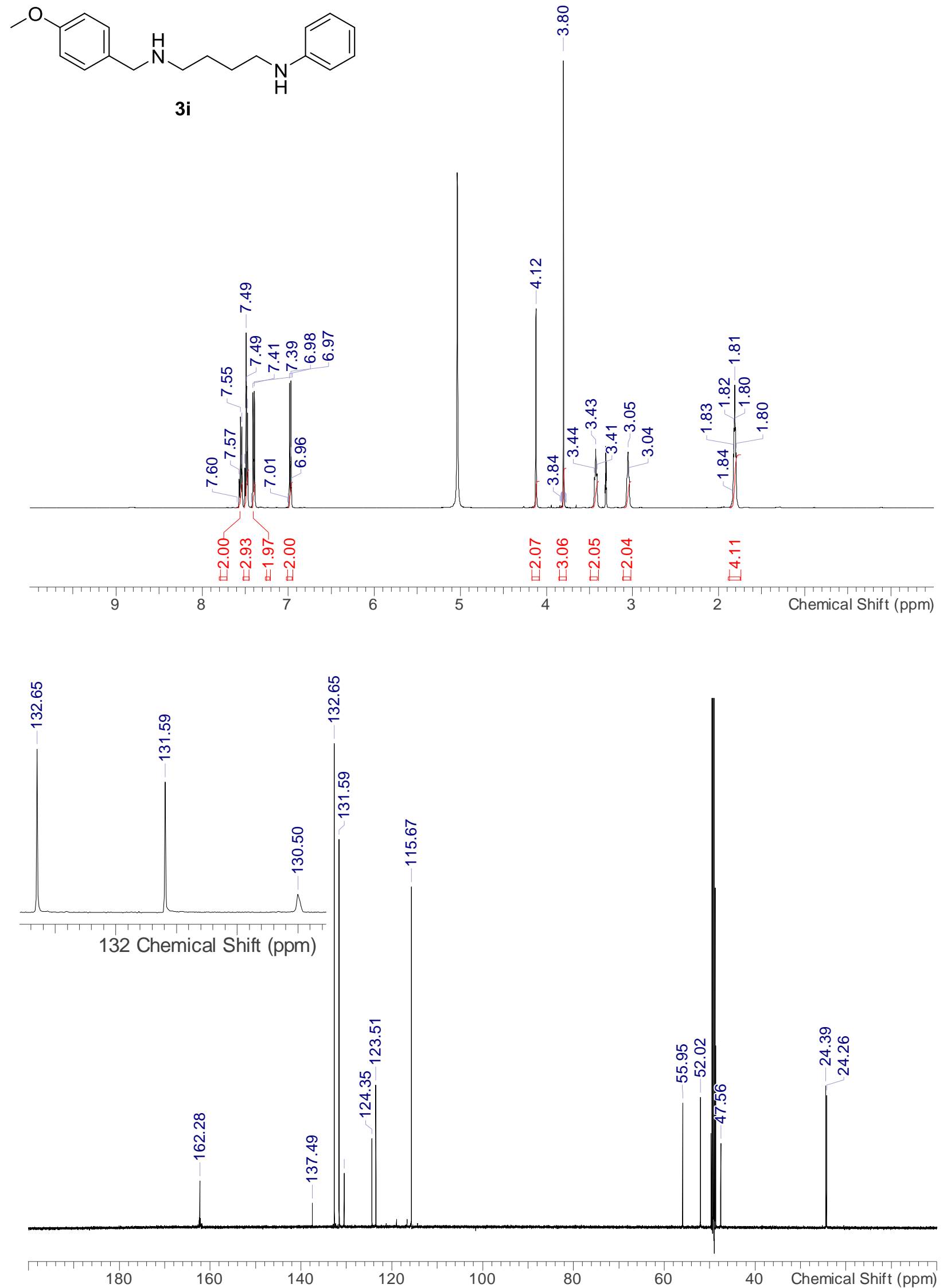
Page S29
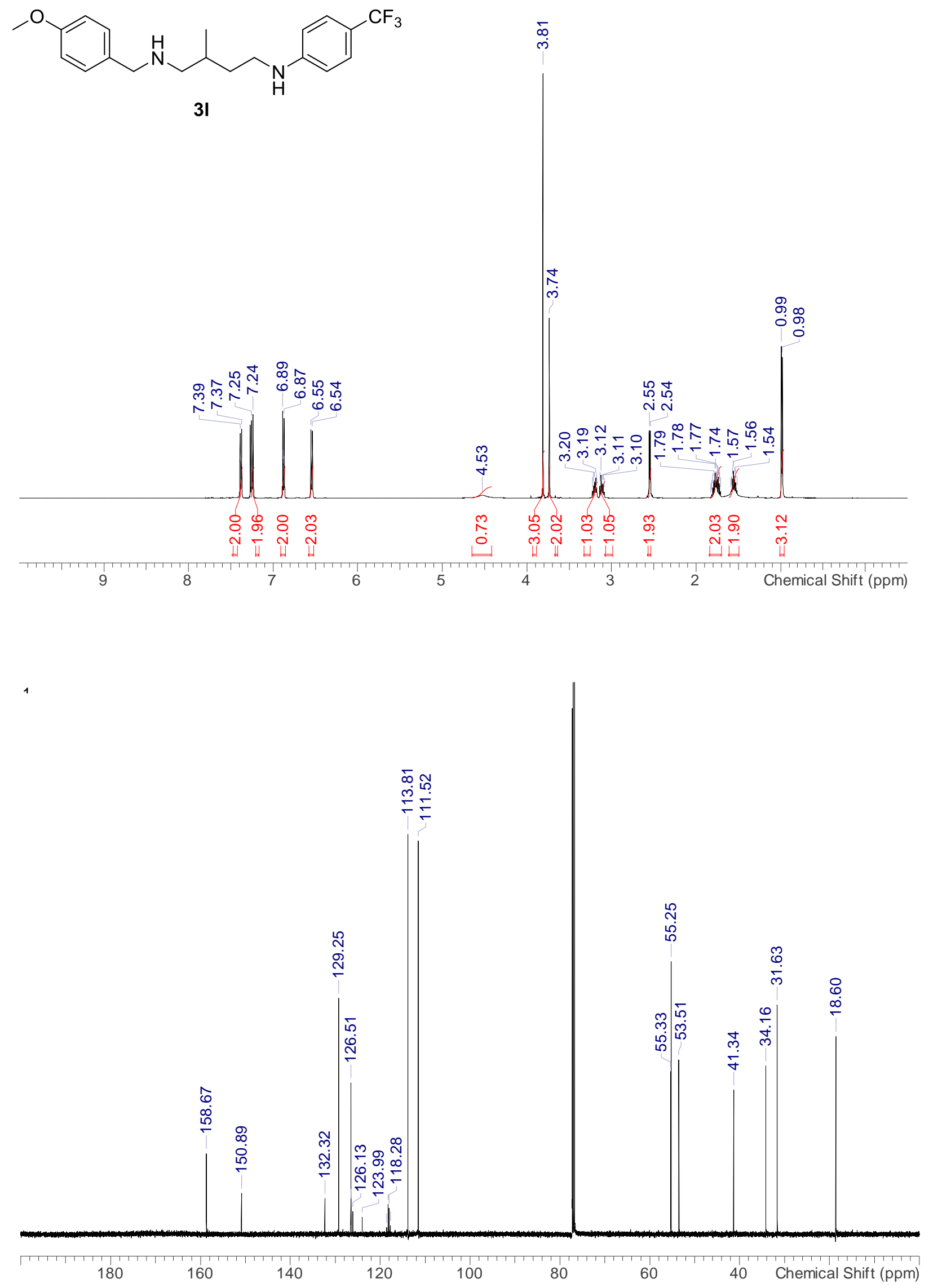

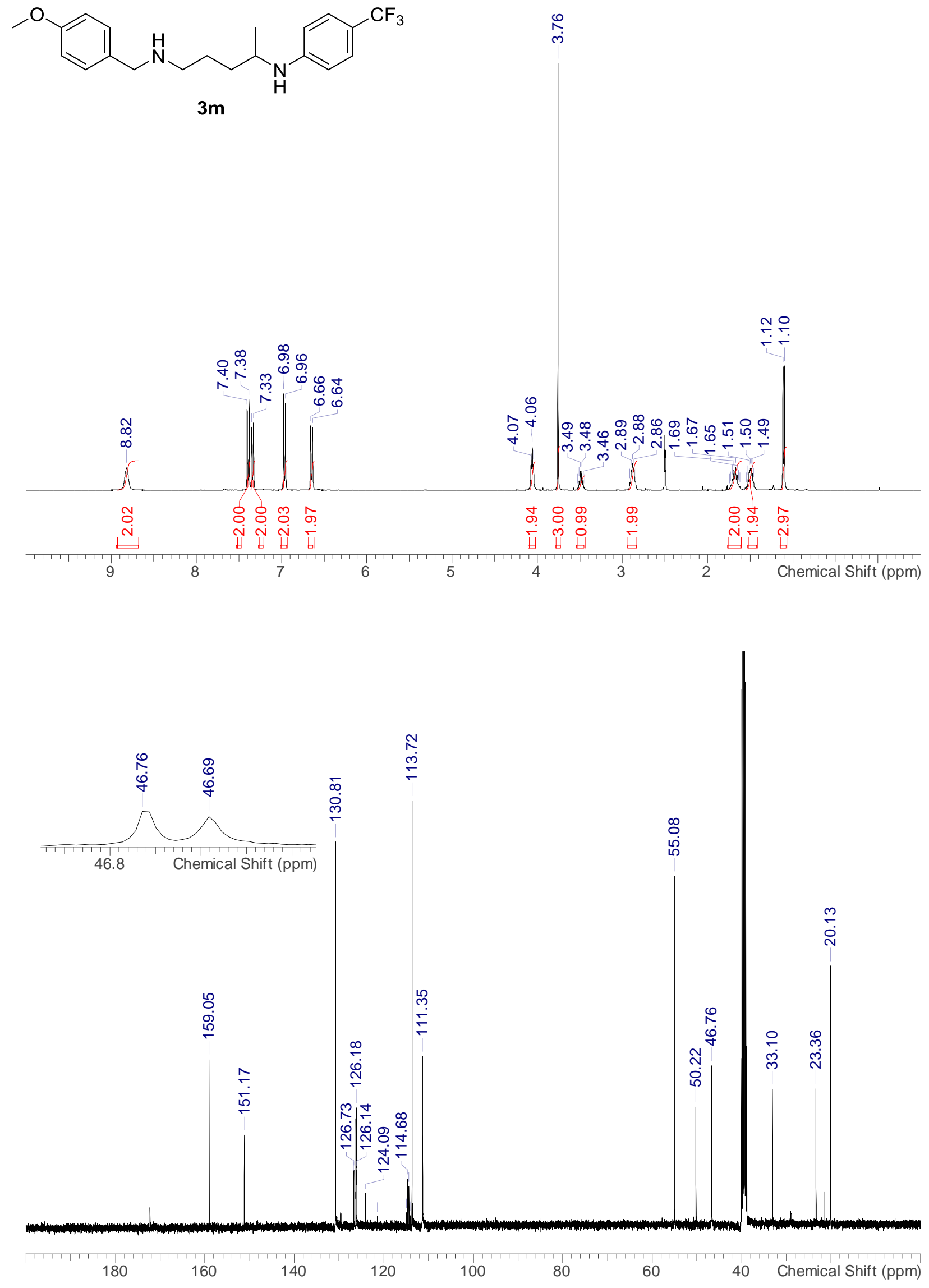
Page S31
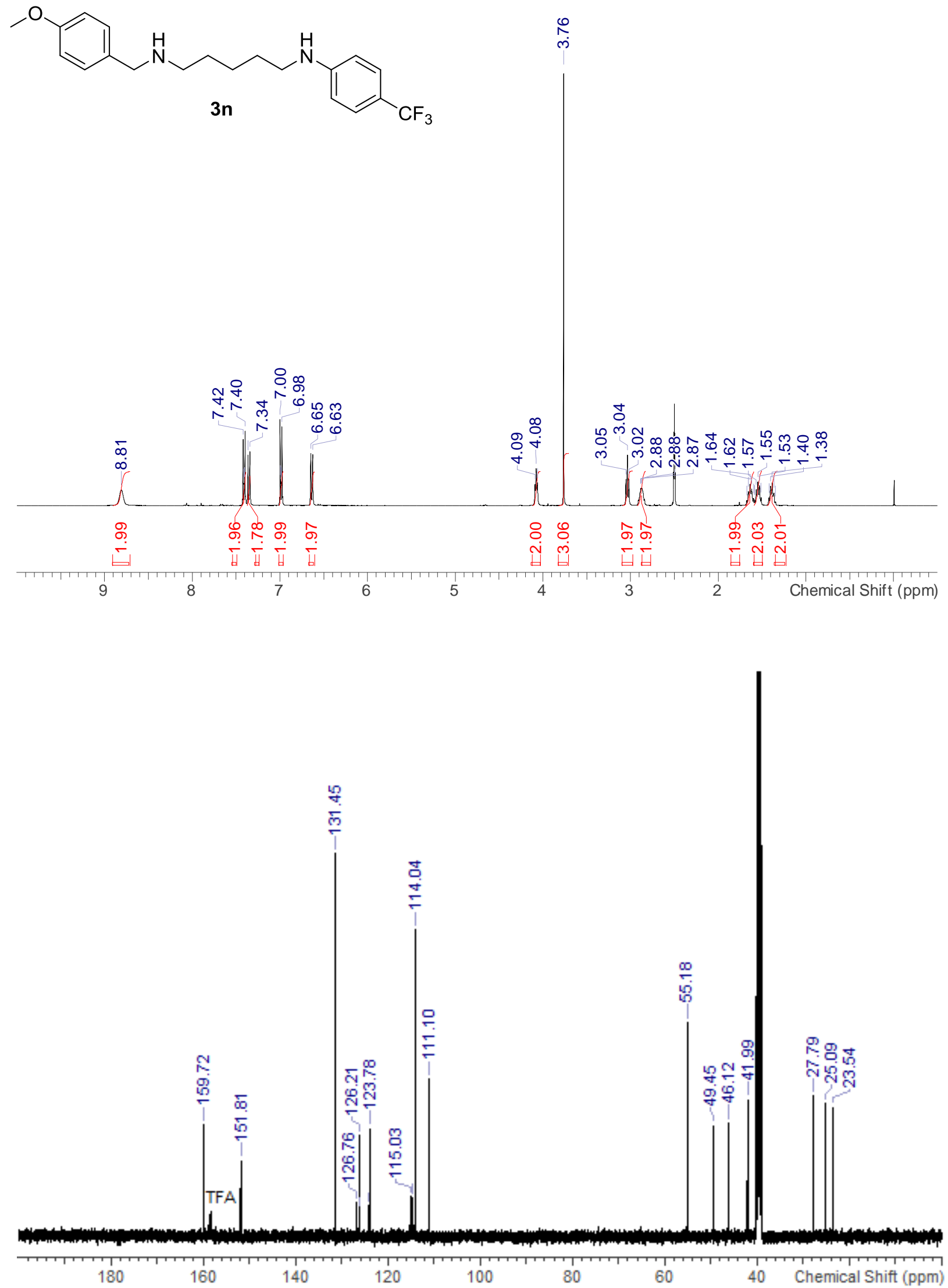
Page S32
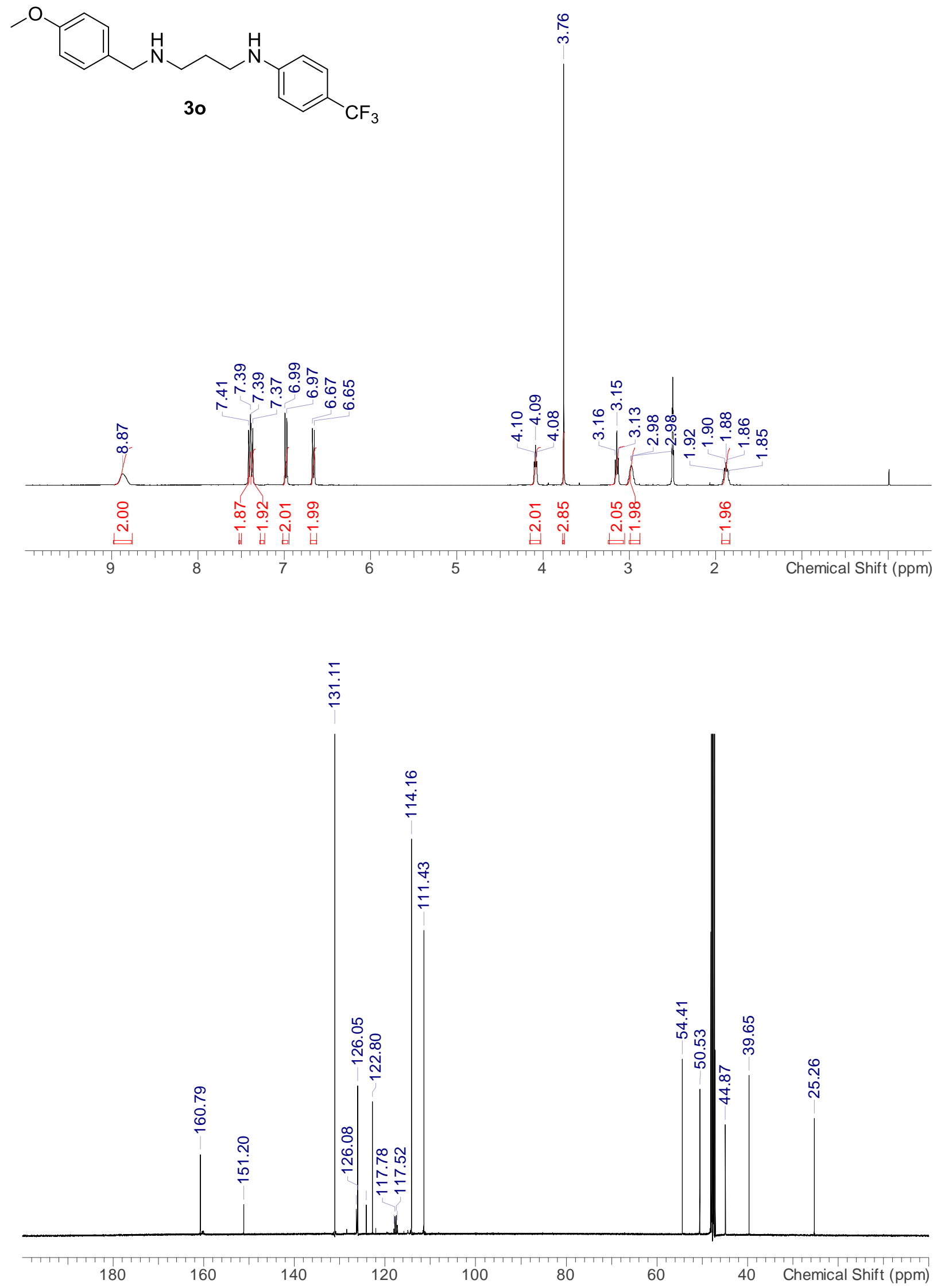
Page S33
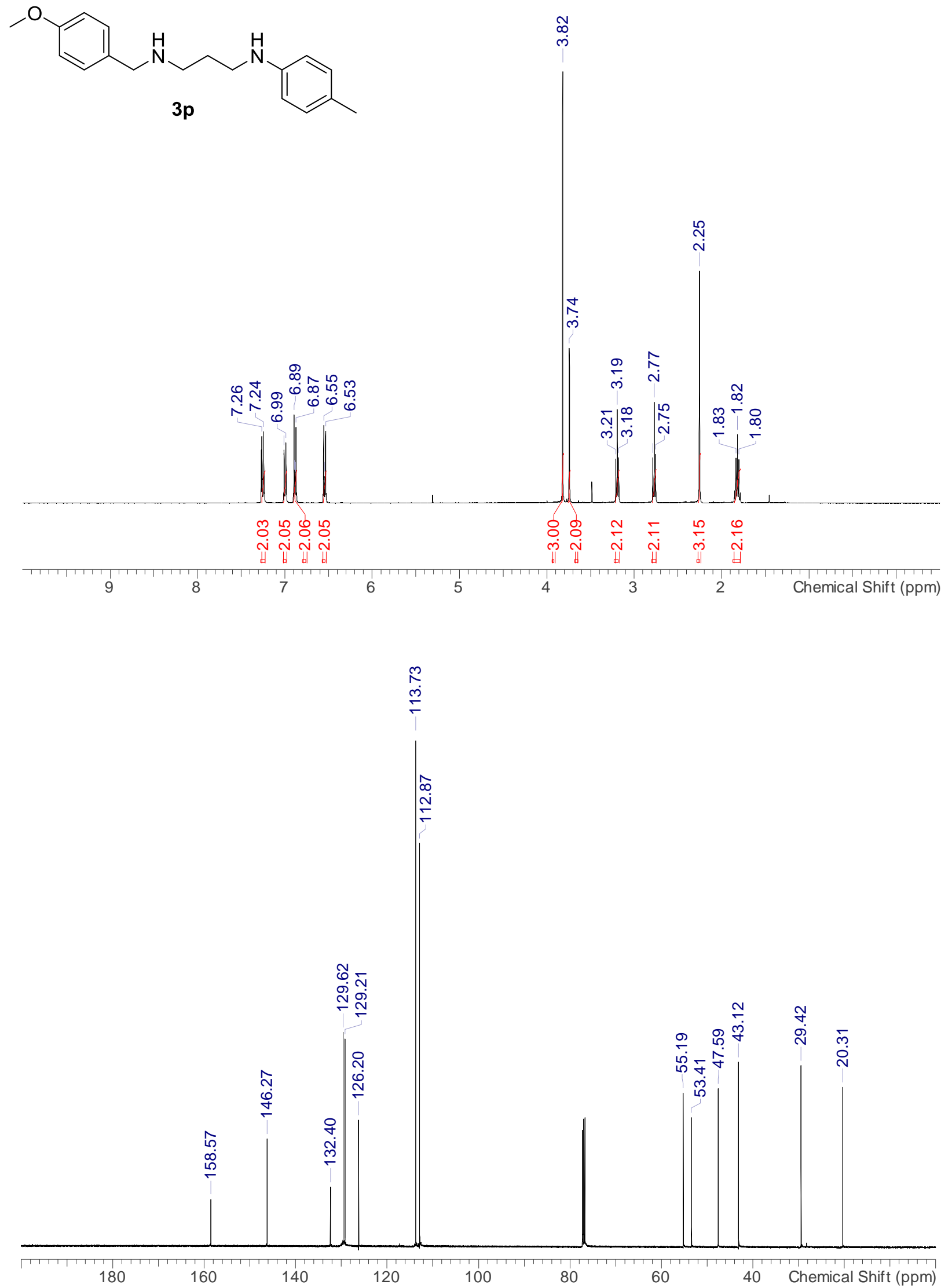
Page S34
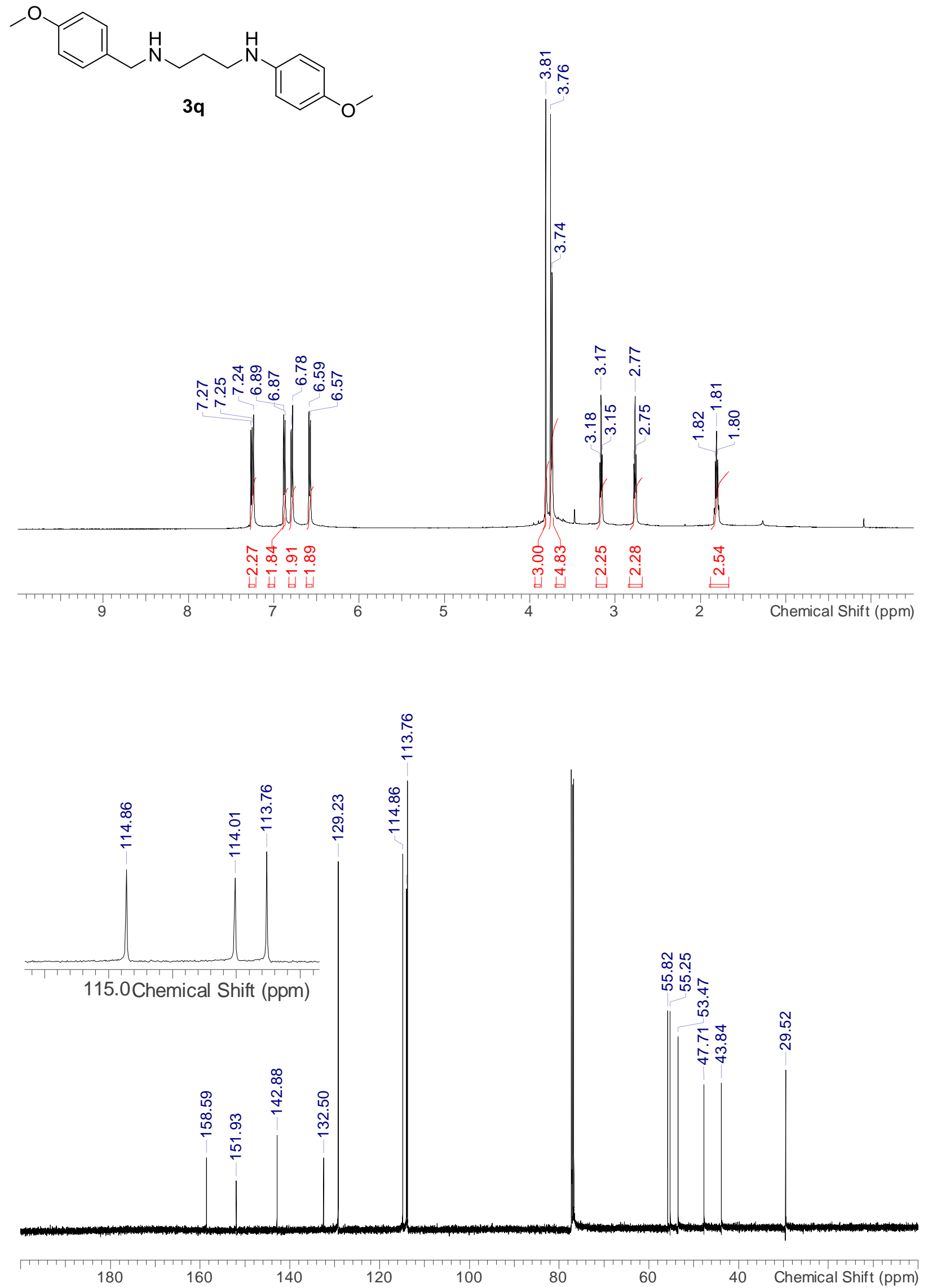
<smiles>FC(F)(F)c1ccc(NCCCCNCC2CCCCC2)cc1</smiles>

$5 a$
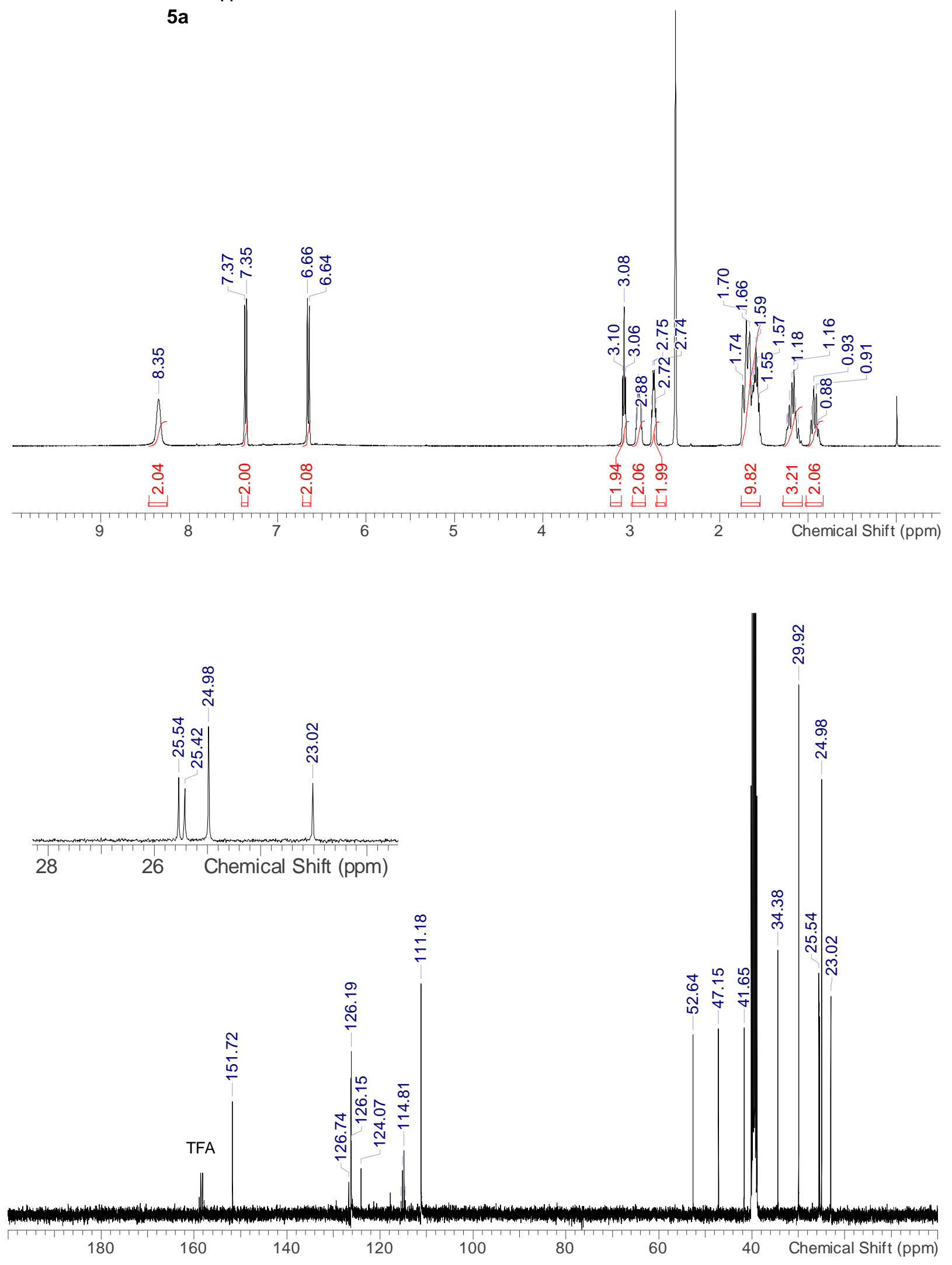
Page S36<smiles>FC(F)(F)c1ccc(NCCCCNC2CCCCC2)cc1</smiles>
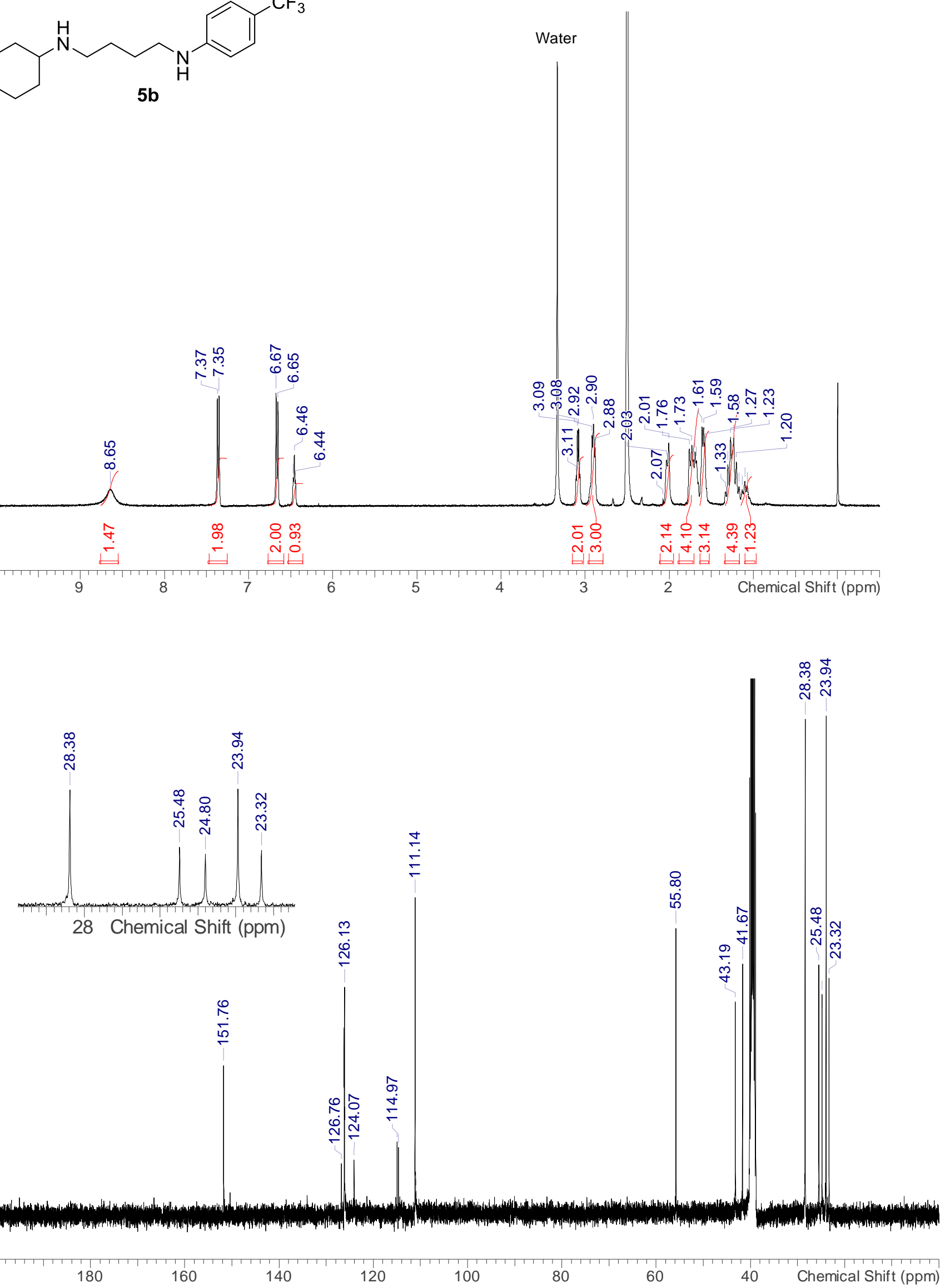
Page S37

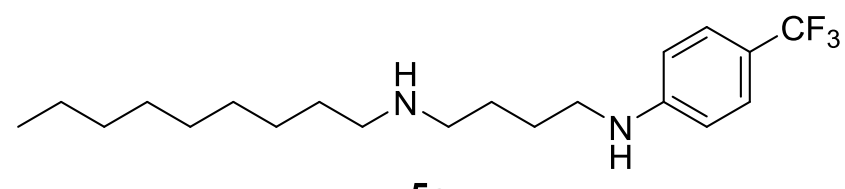

$5 c$
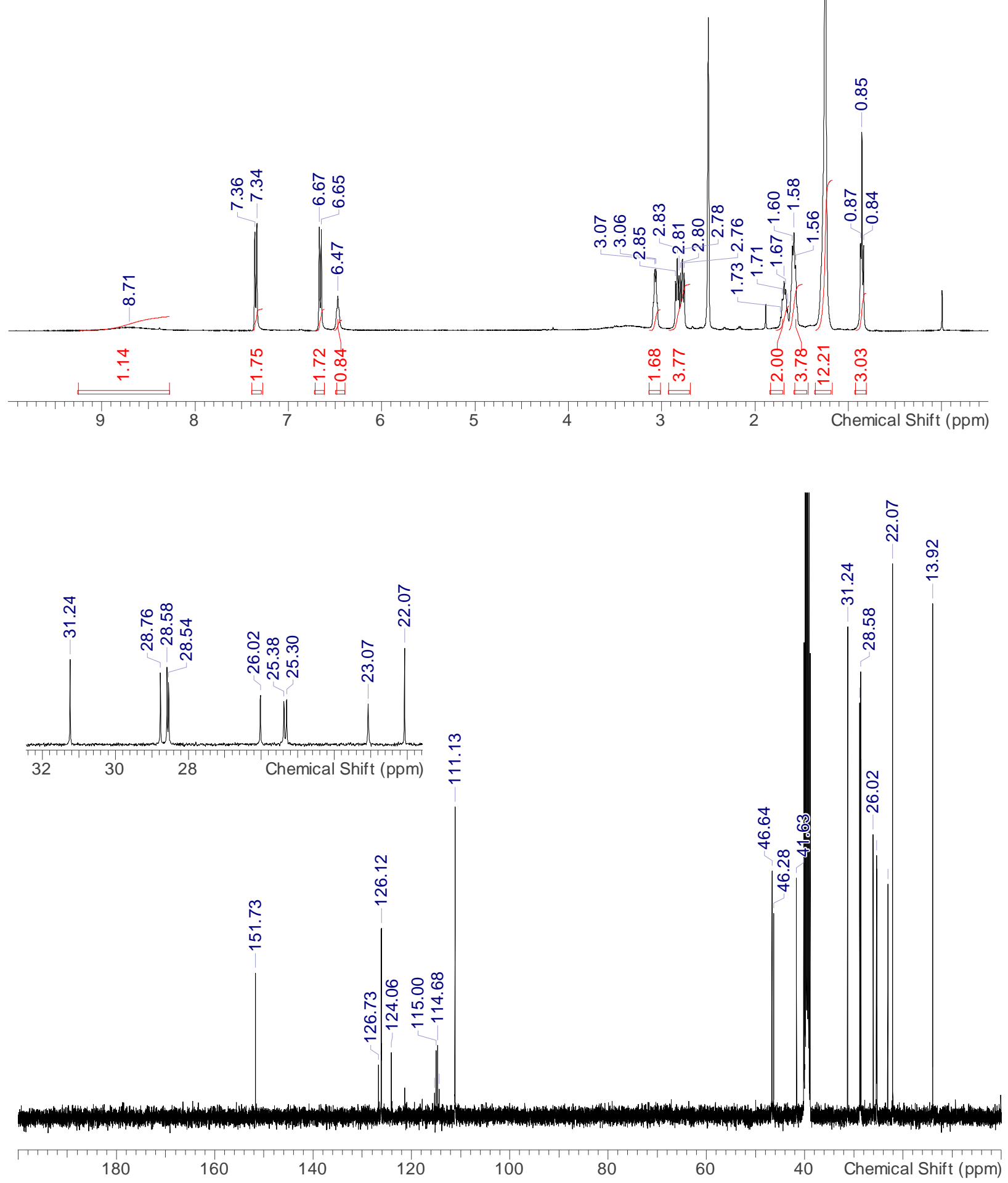
Page S38<smiles>COC[C@H](C)NCCCCNc1ccc(C(F)(F)F)cc1</smiles>
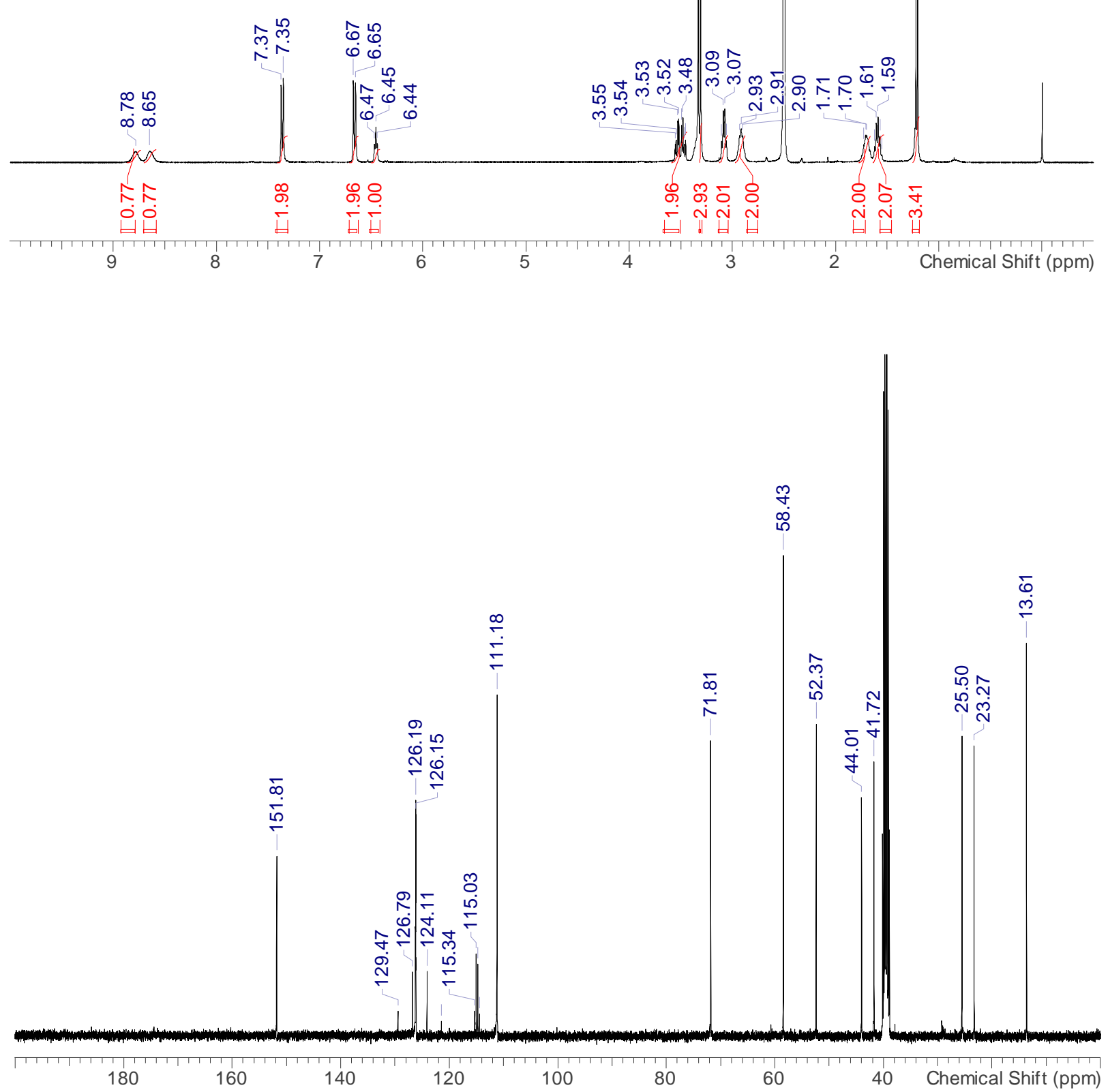
Page S39<smiles>C[C@H](NCCCCNc1ccc(C(F)(F)F)cc1)c1ccccc1</smiles>
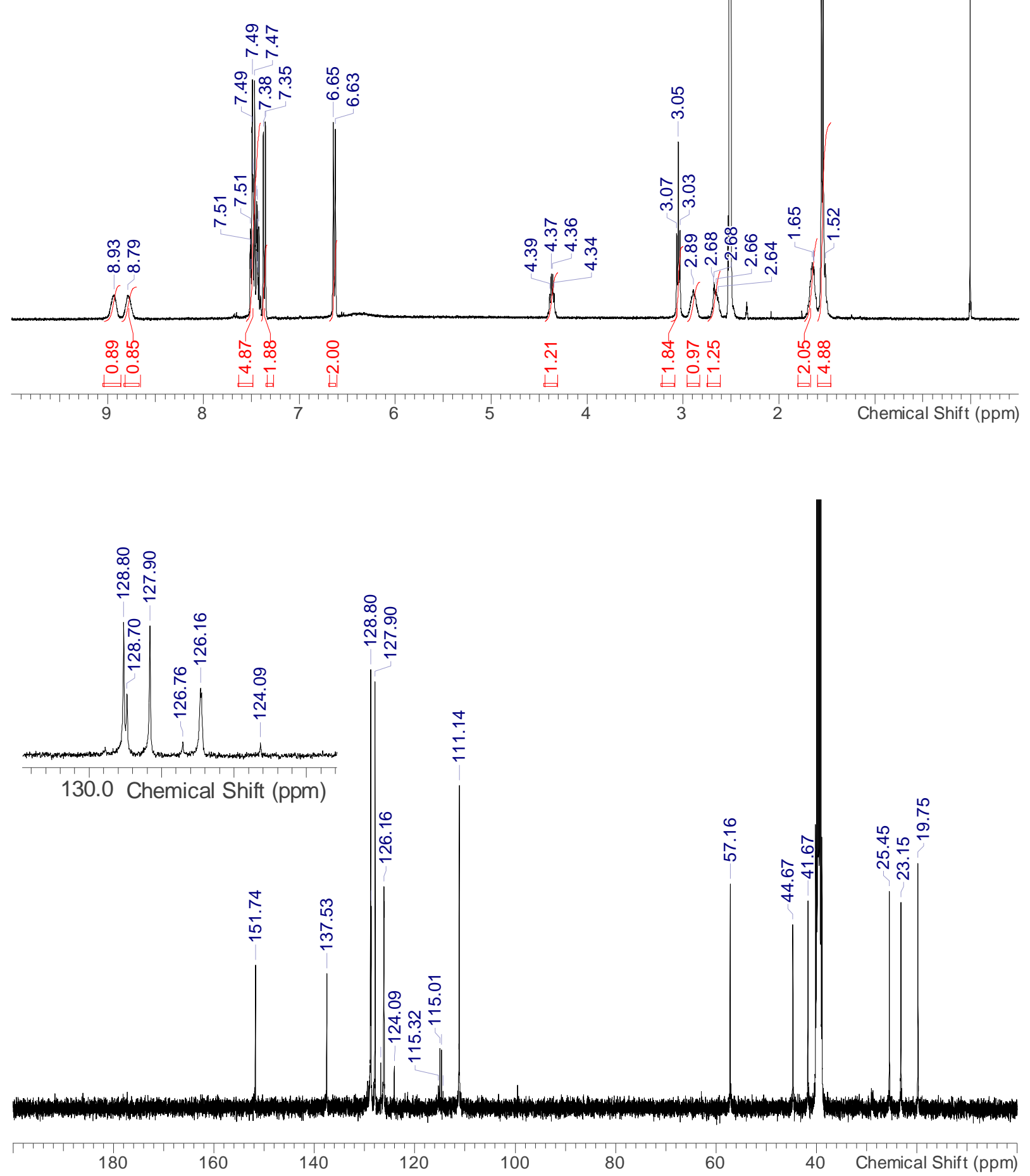
Page S40
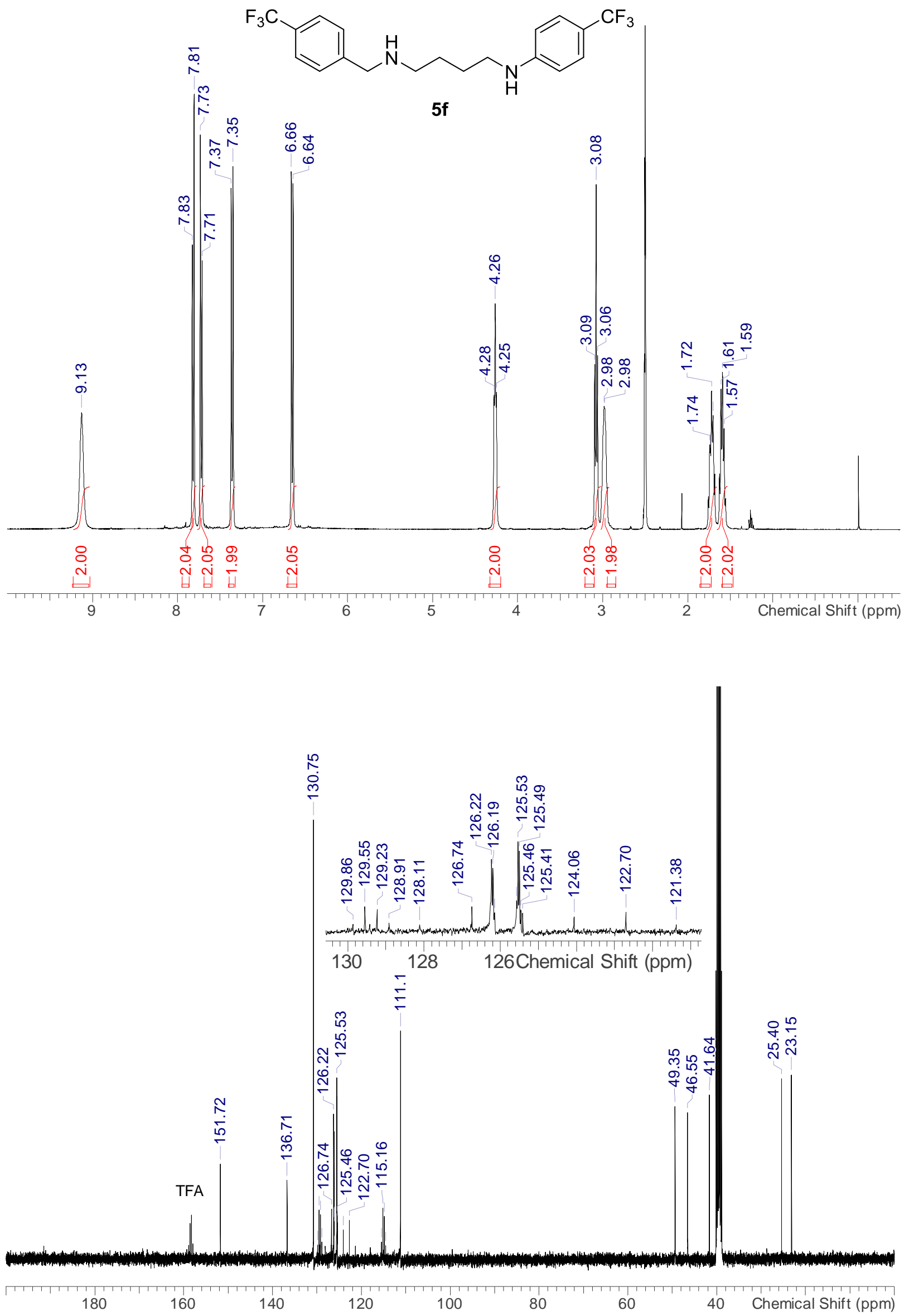
Page S41<smiles>COc1ccc(NCCCCNc2ccc(C(F)(F)F)cc2)cc1</smiles>
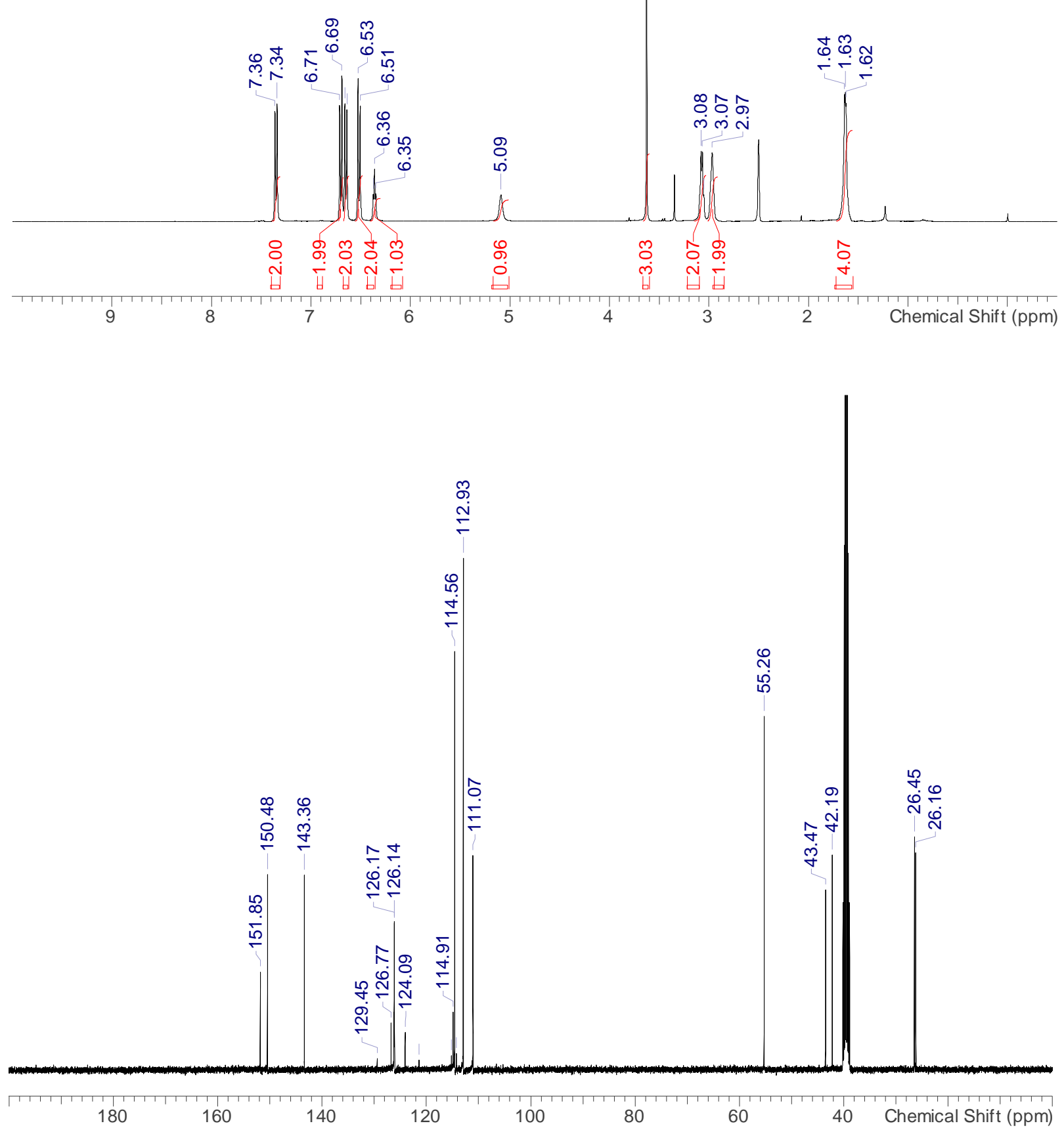
Page S42<smiles>CC(C)(C)OC(=O)N1CCN(CCCCNc2ccc(C(F)(F)F)cc2)CC1</smiles>

$6 a$
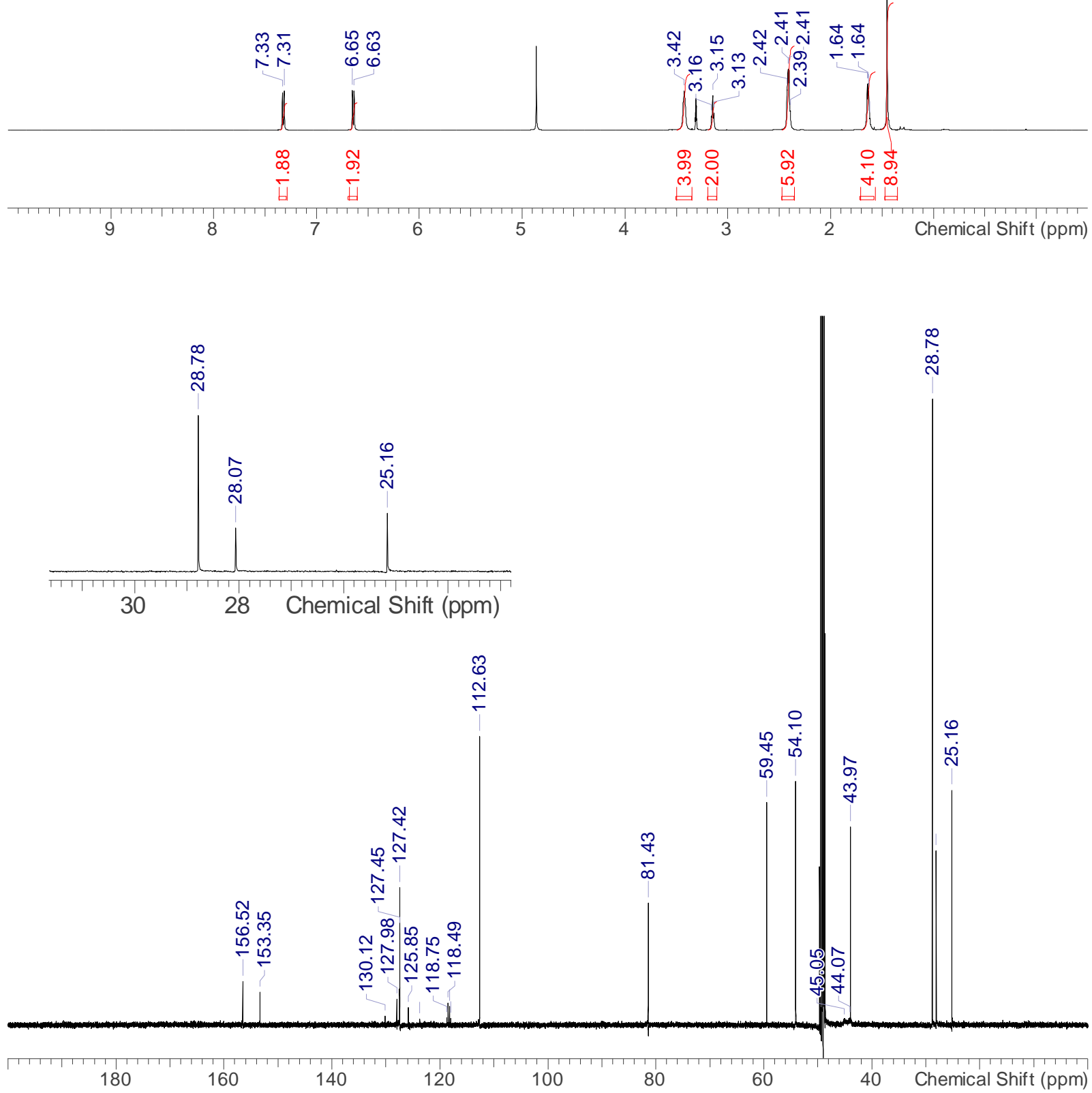
Page S43
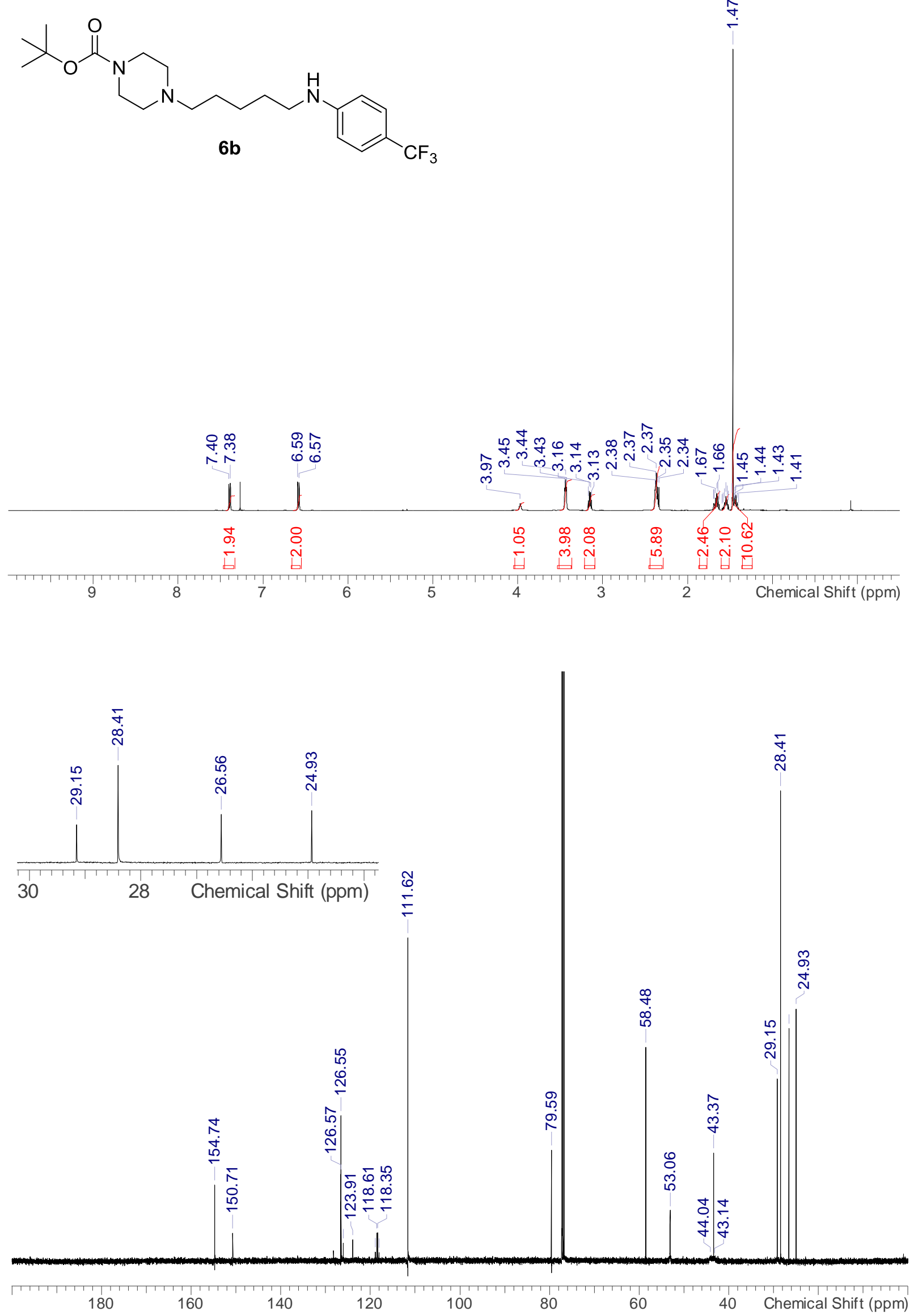
Page S44
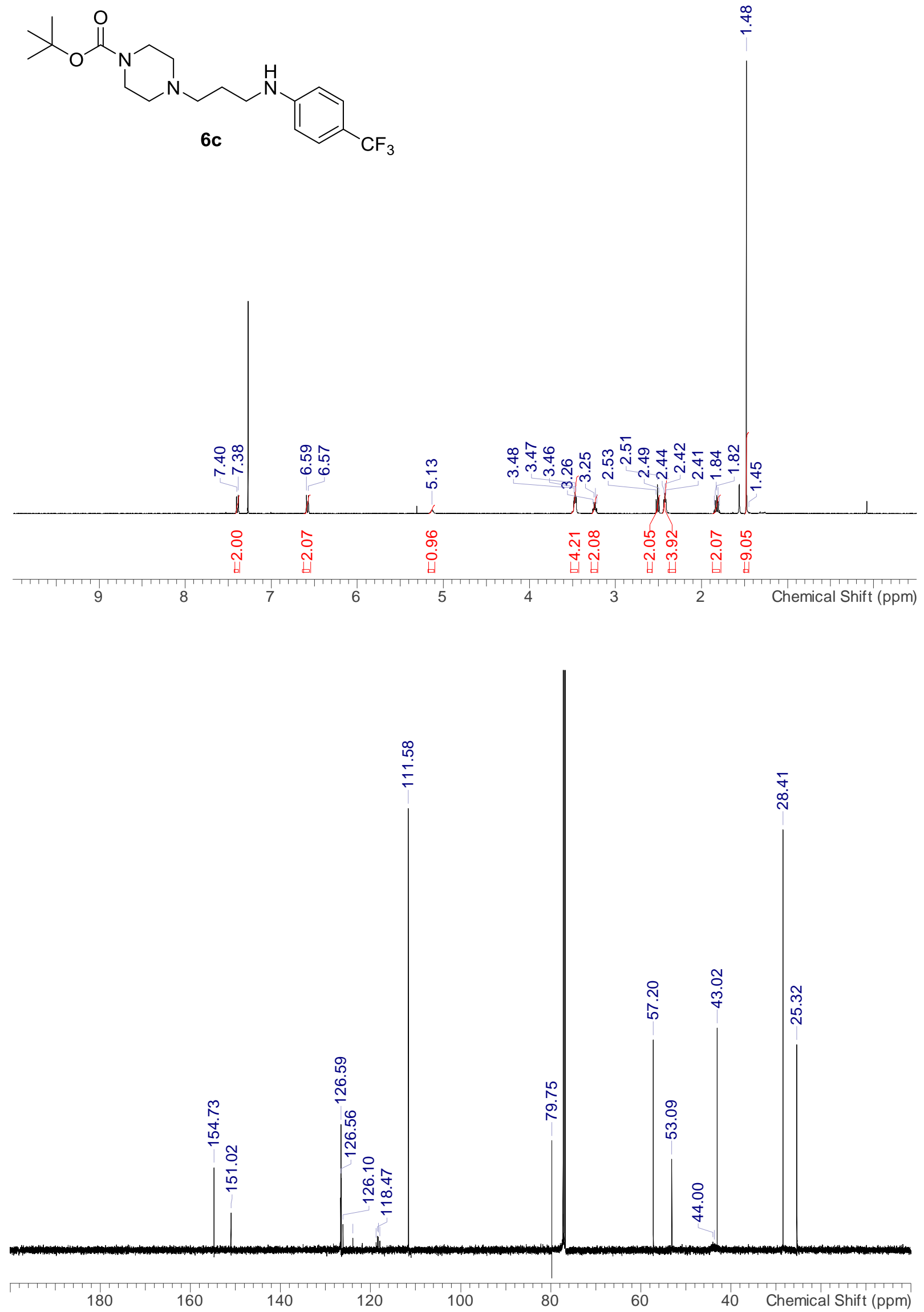
Page S45
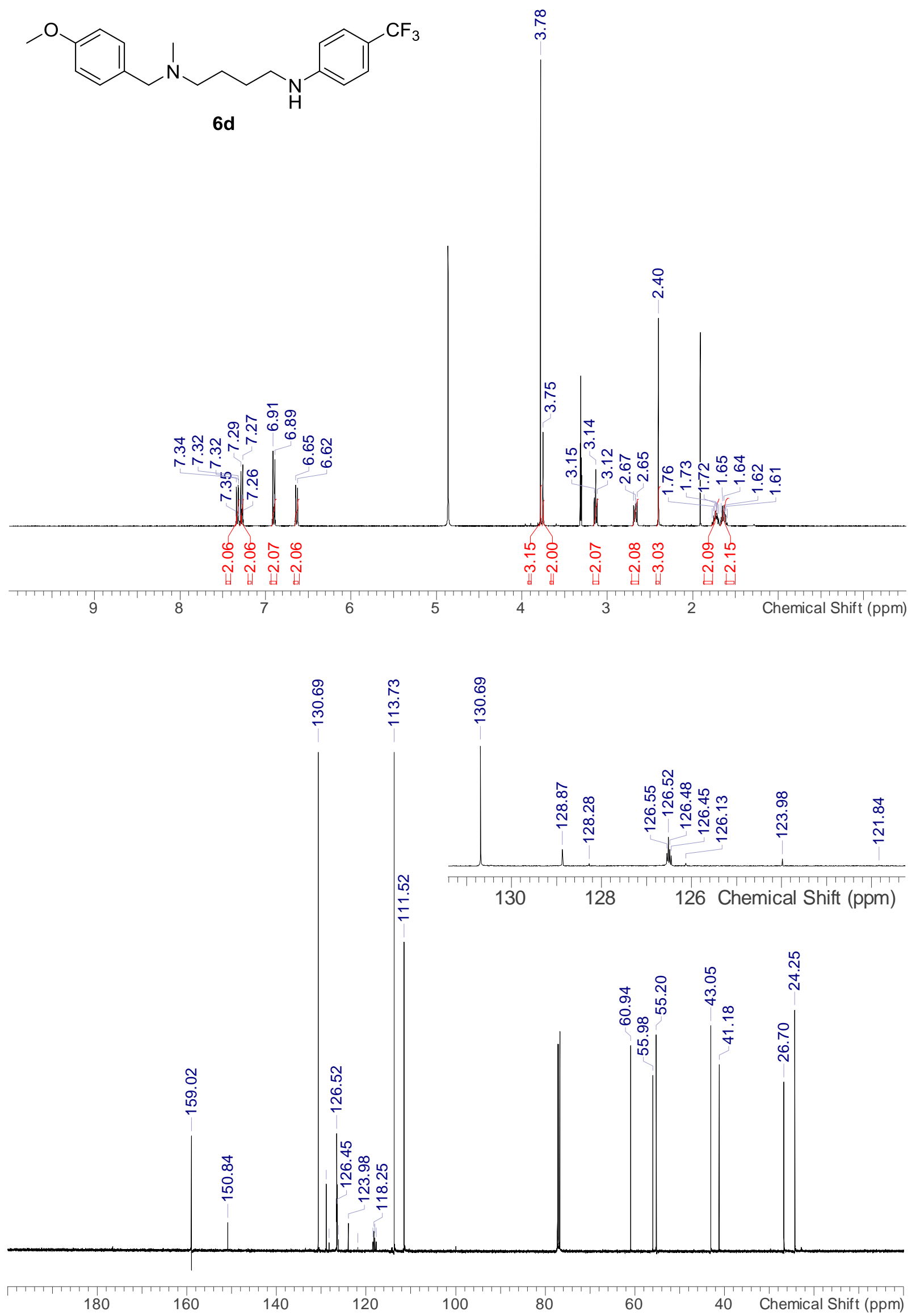
Page S46
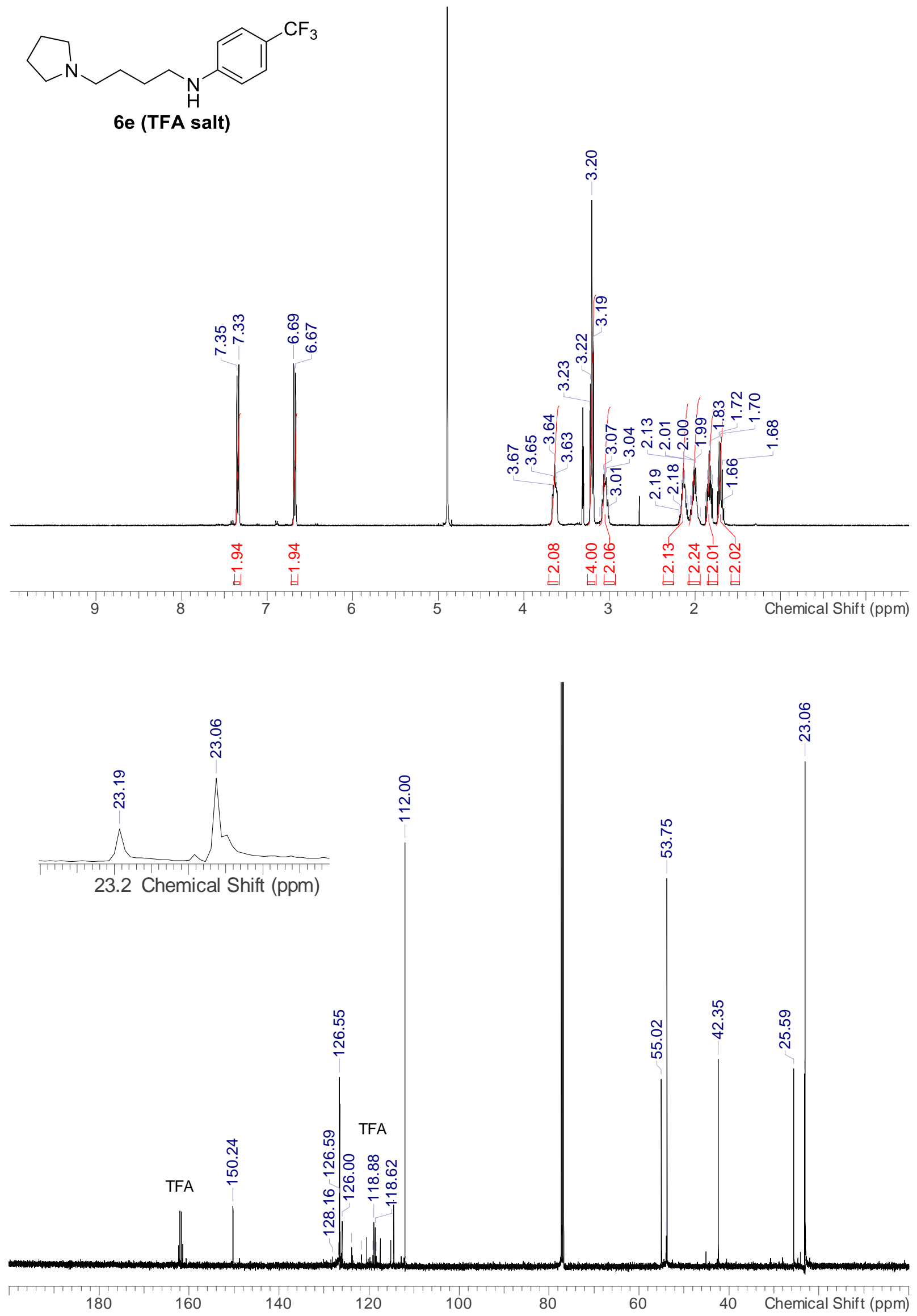
Page S47

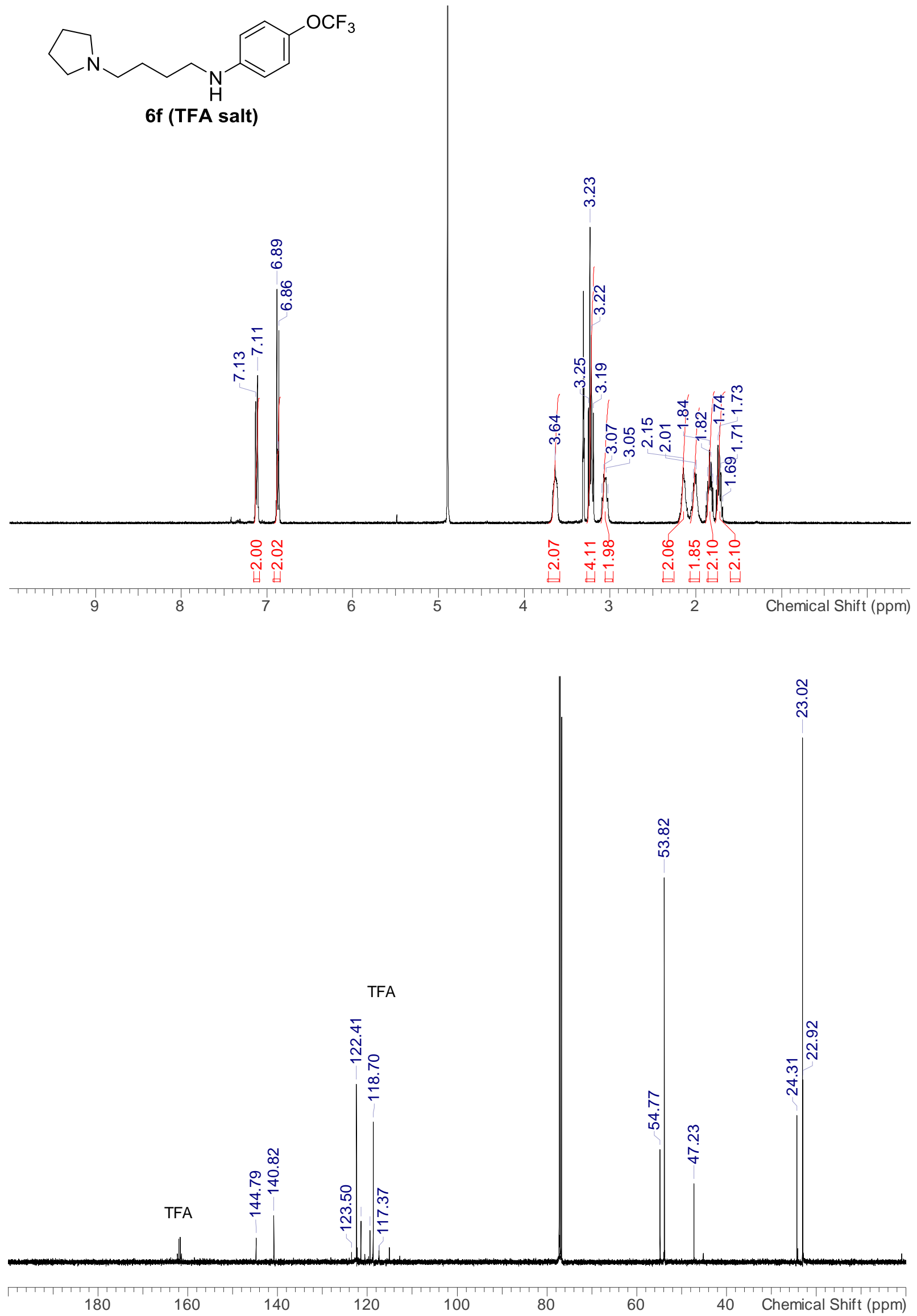


Page S48<smiles>FC(F)(F)c1ccc(NCCCN2CCCC2)cc1</smiles>
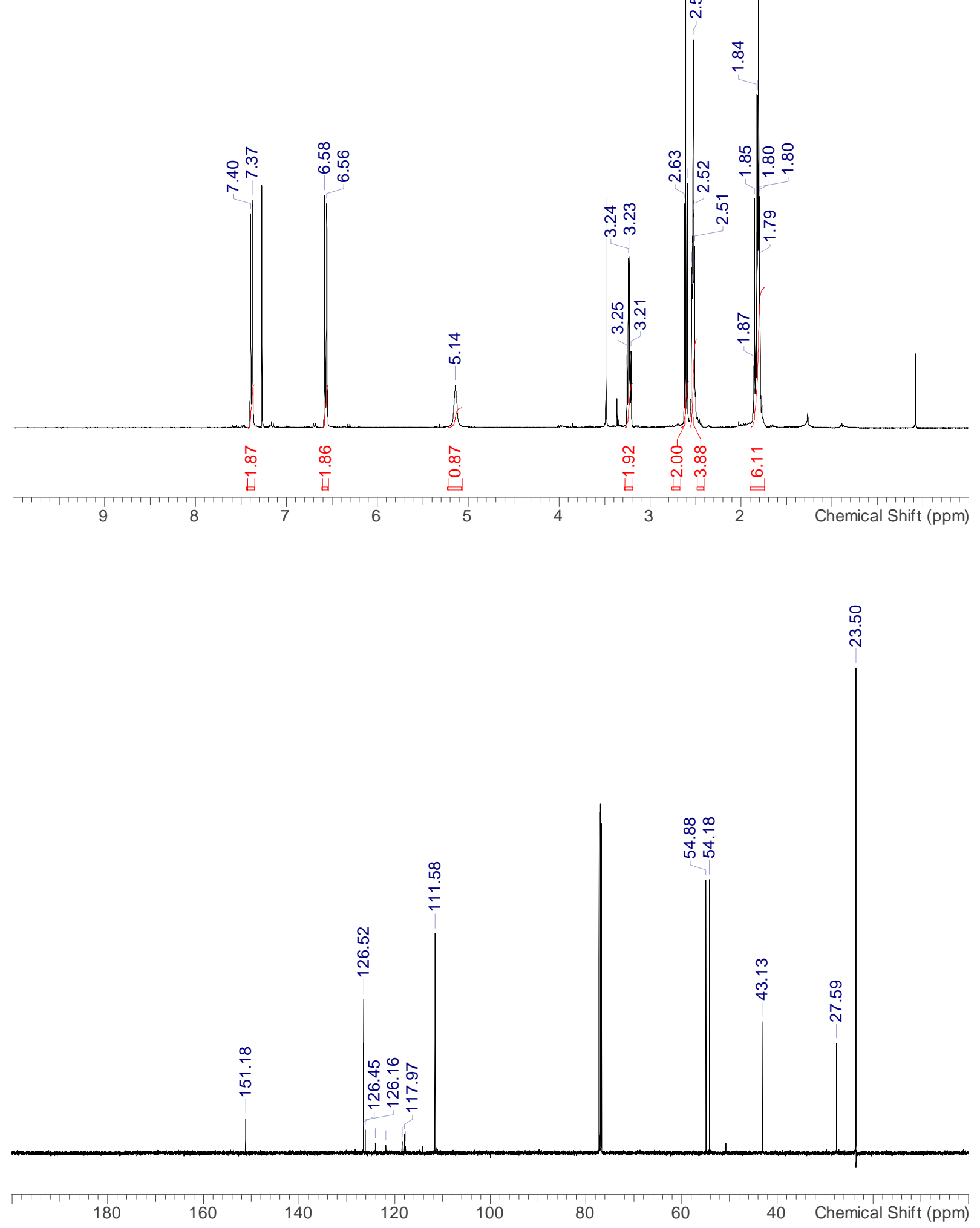
Page S49<smiles>CCOC(=O)[C@@H]1CCC(=O)N1c1ccc(C(F)(F)F)cc1</smiles>
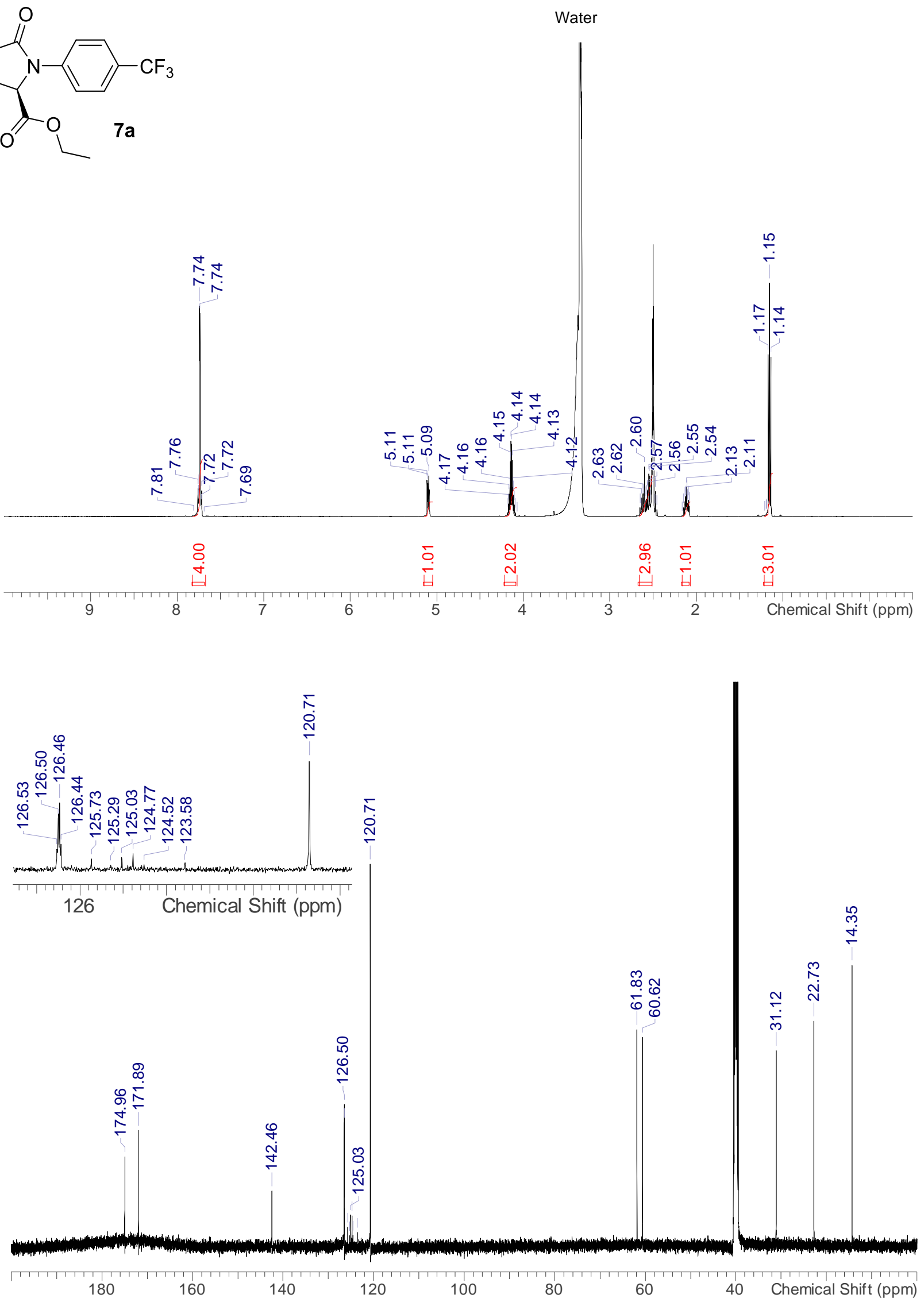
Page S50<smiles>CCOC(=O)[C@@H]1CCC(=O)N1c1cccc(C(F)(F)F)c1</smiles>

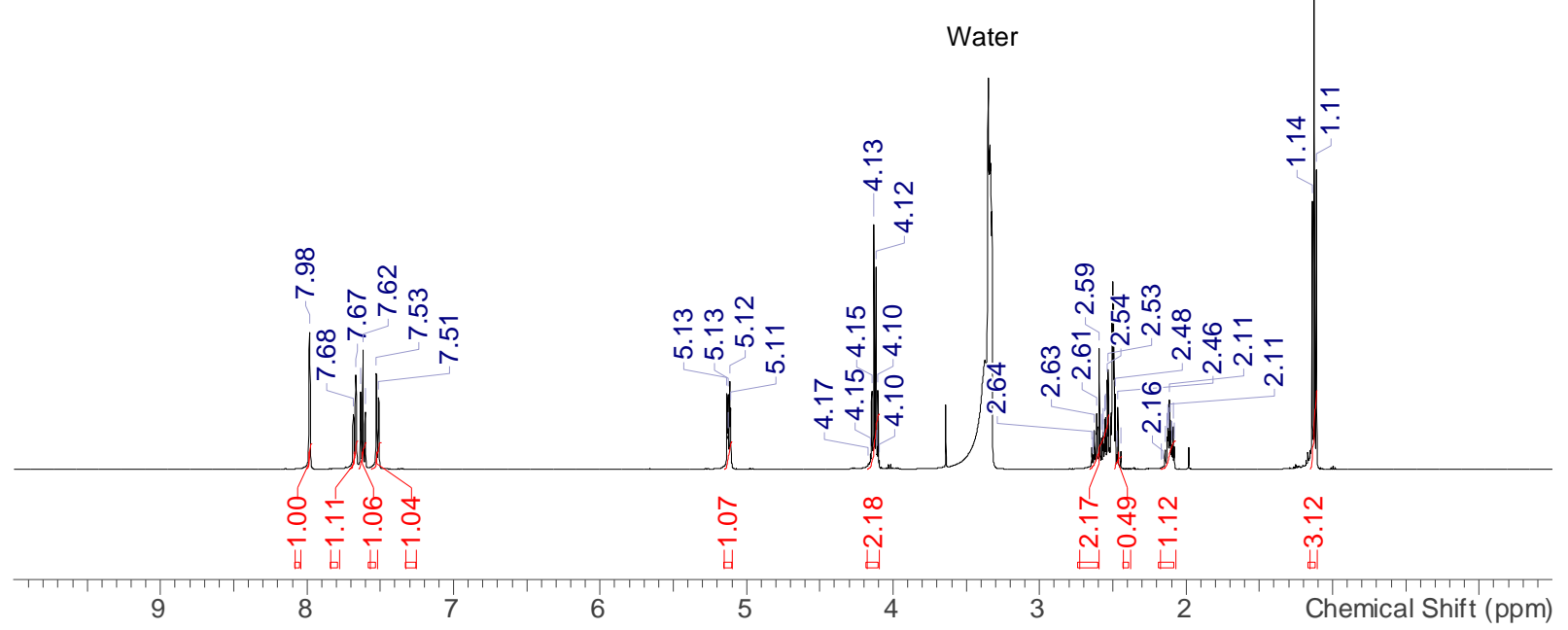

F.
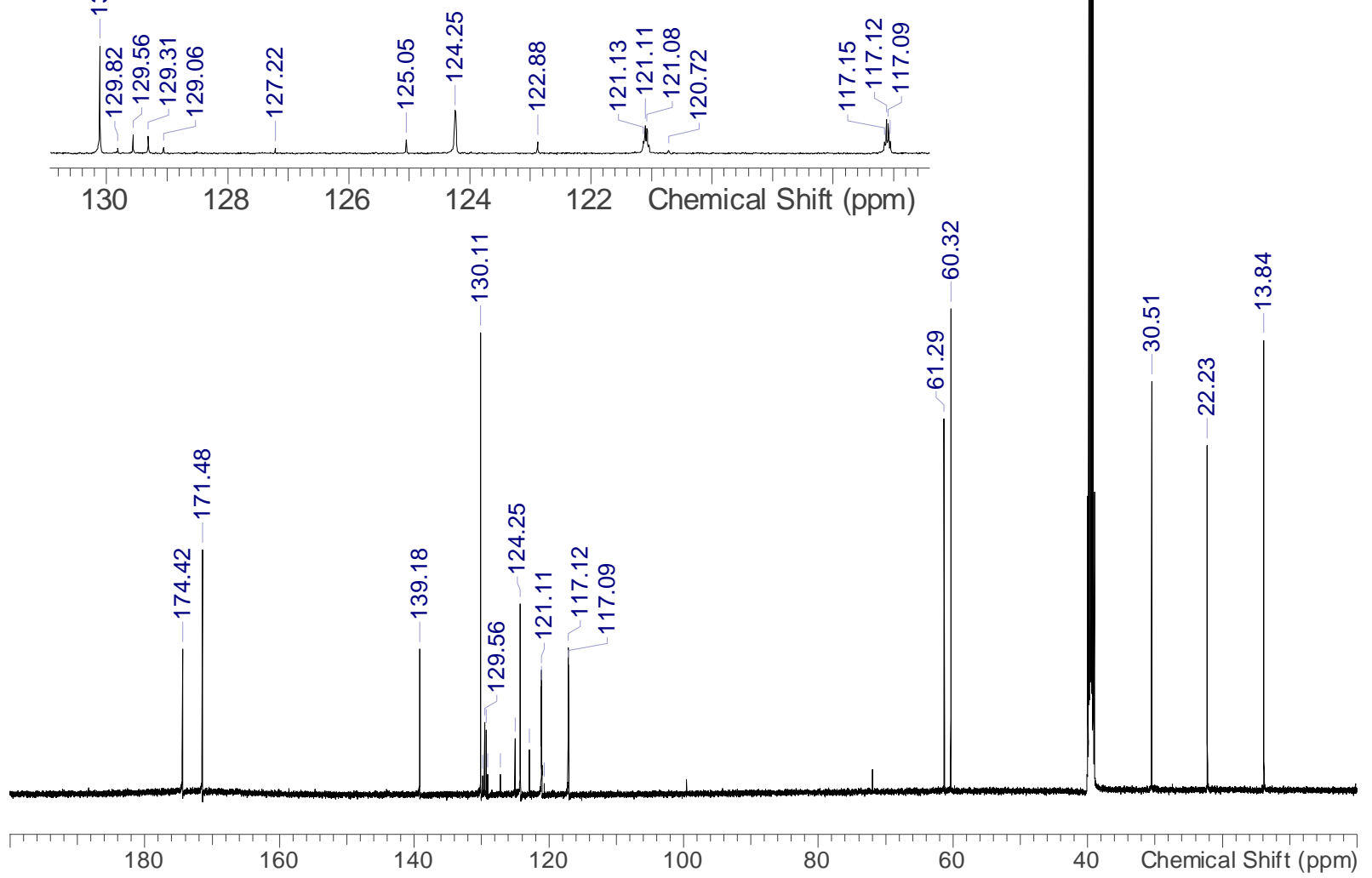
Page S51<smiles>CCOC(=O)[C@@H]1CCC(=O)N1c1cc(F)cc(C(F)(F)F)c1</smiles>
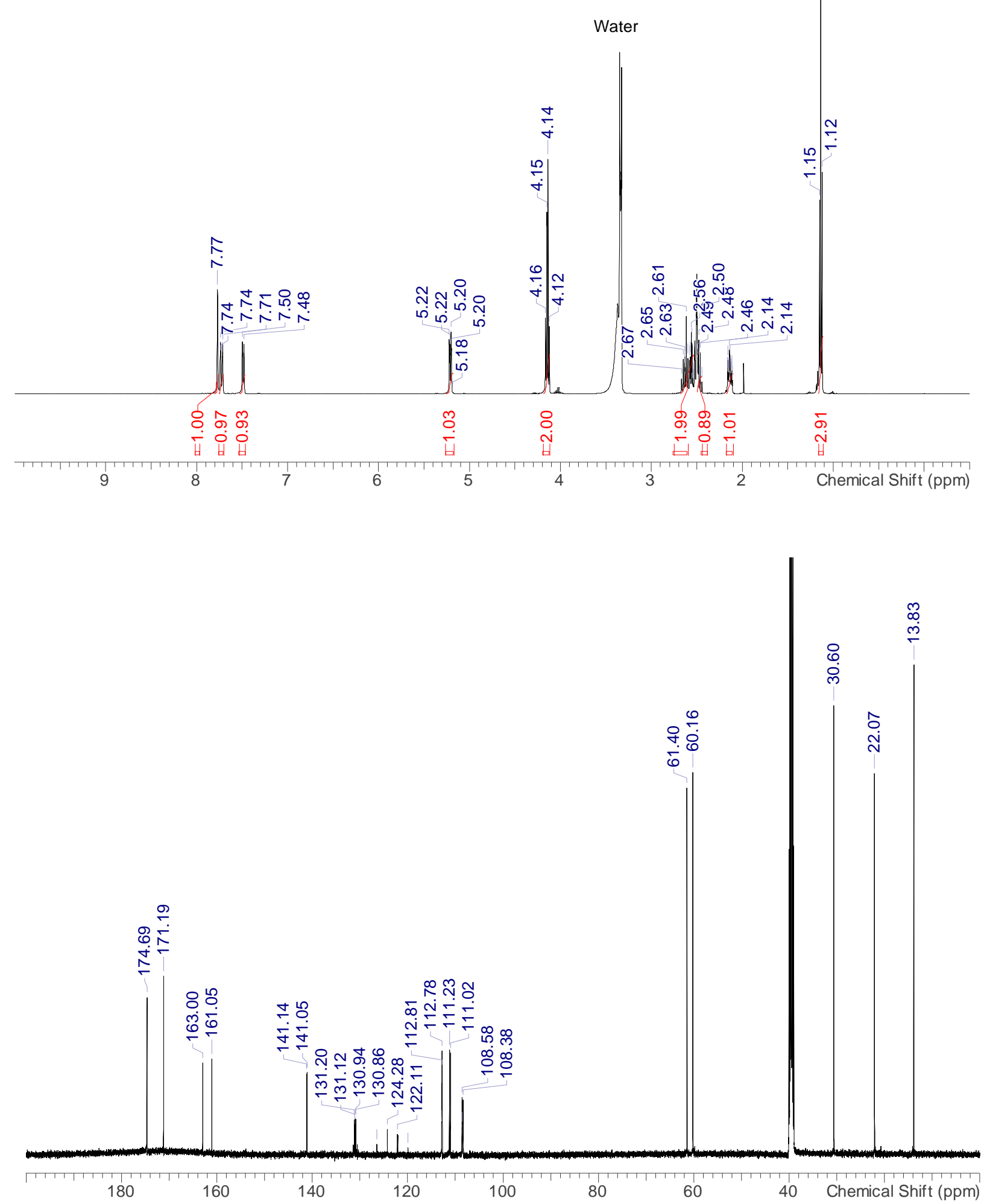
Page S52<smiles>CCOC(=O)[C@@H]1CCC(=O)N1c1cc(F)cc(Cl)c1</smiles>
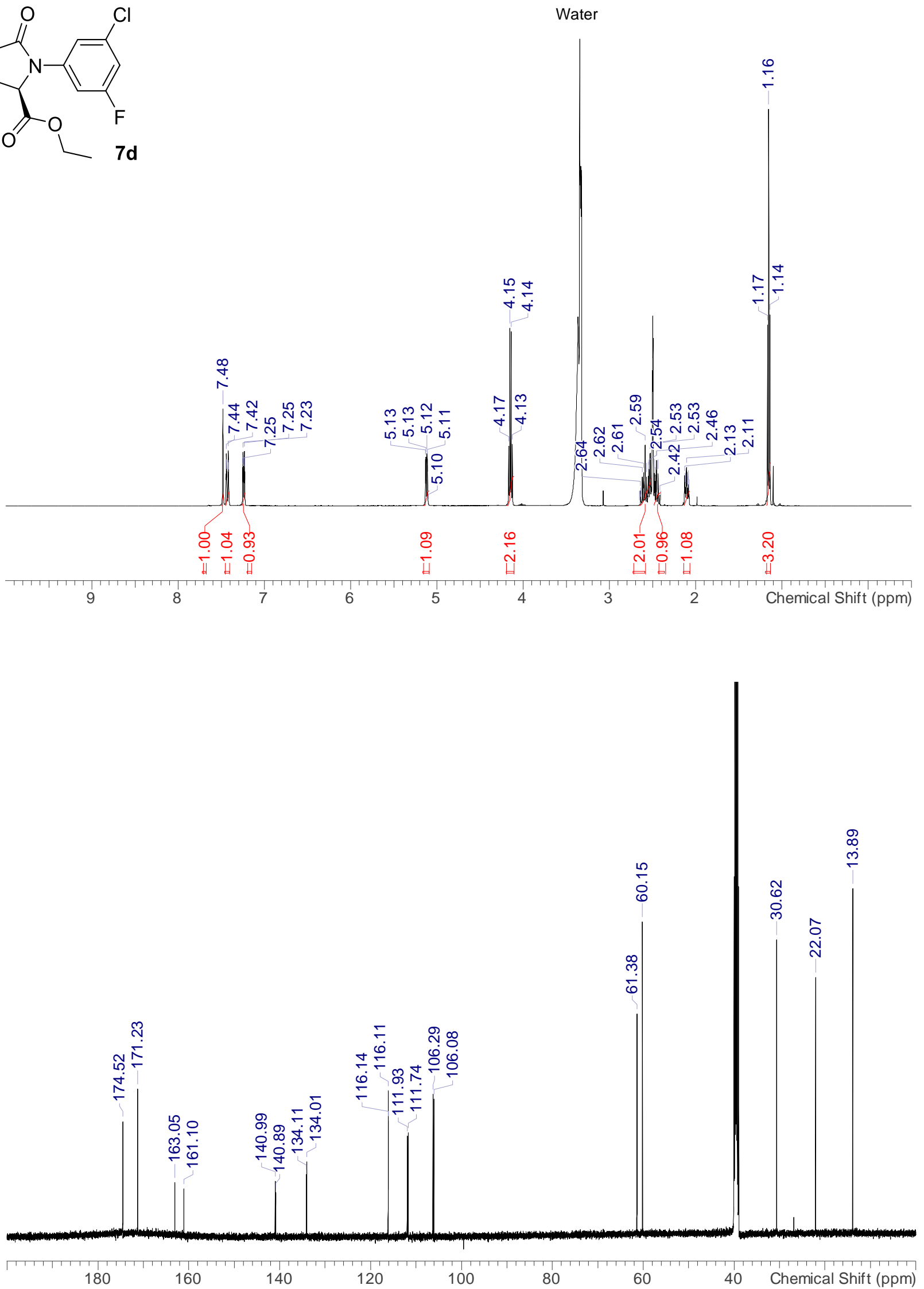
Page S53
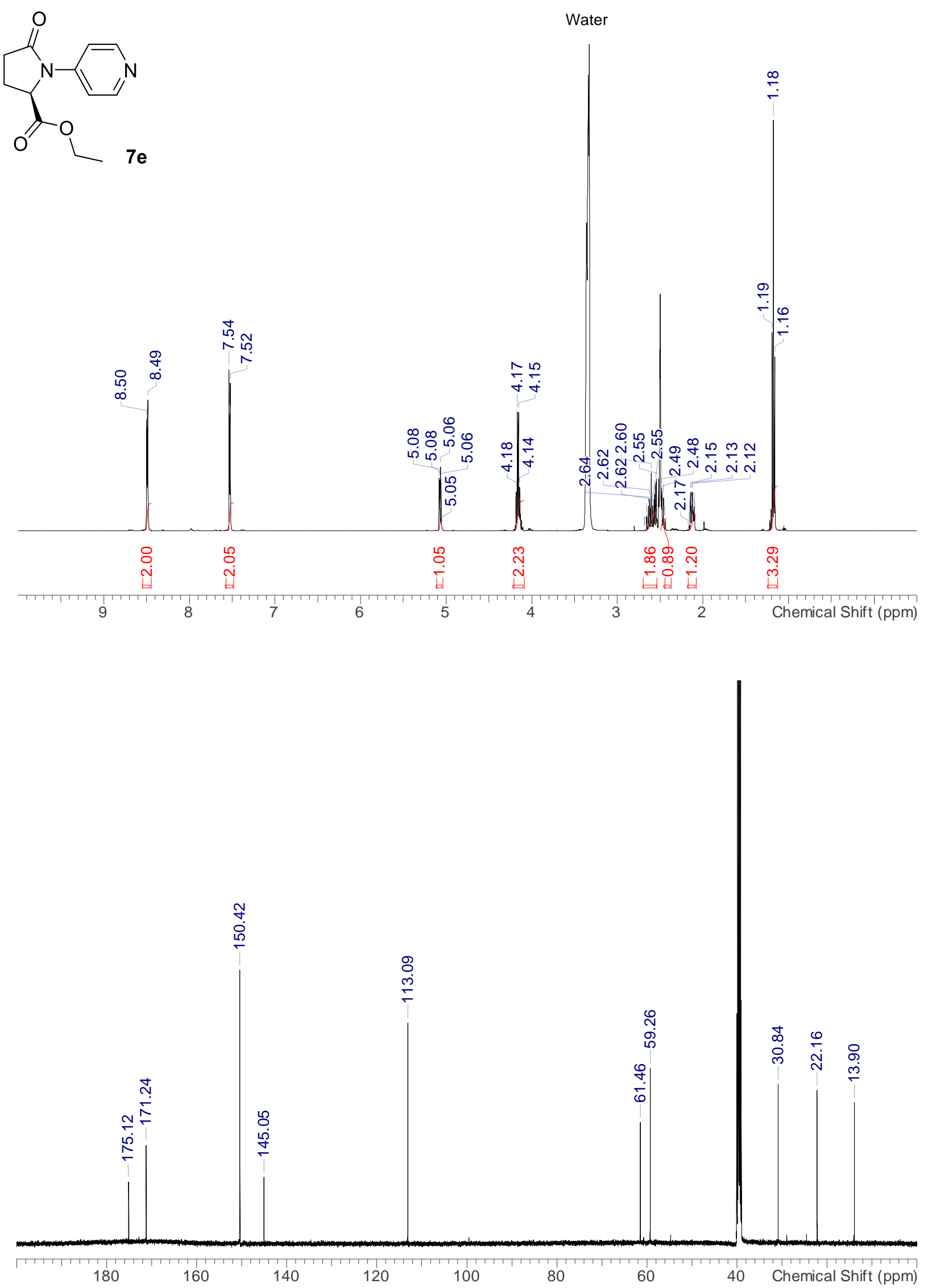
Page S54<smiles>CCOC(=O)[C@@H]1CCC(=O)N1c1cccnc1</smiles>
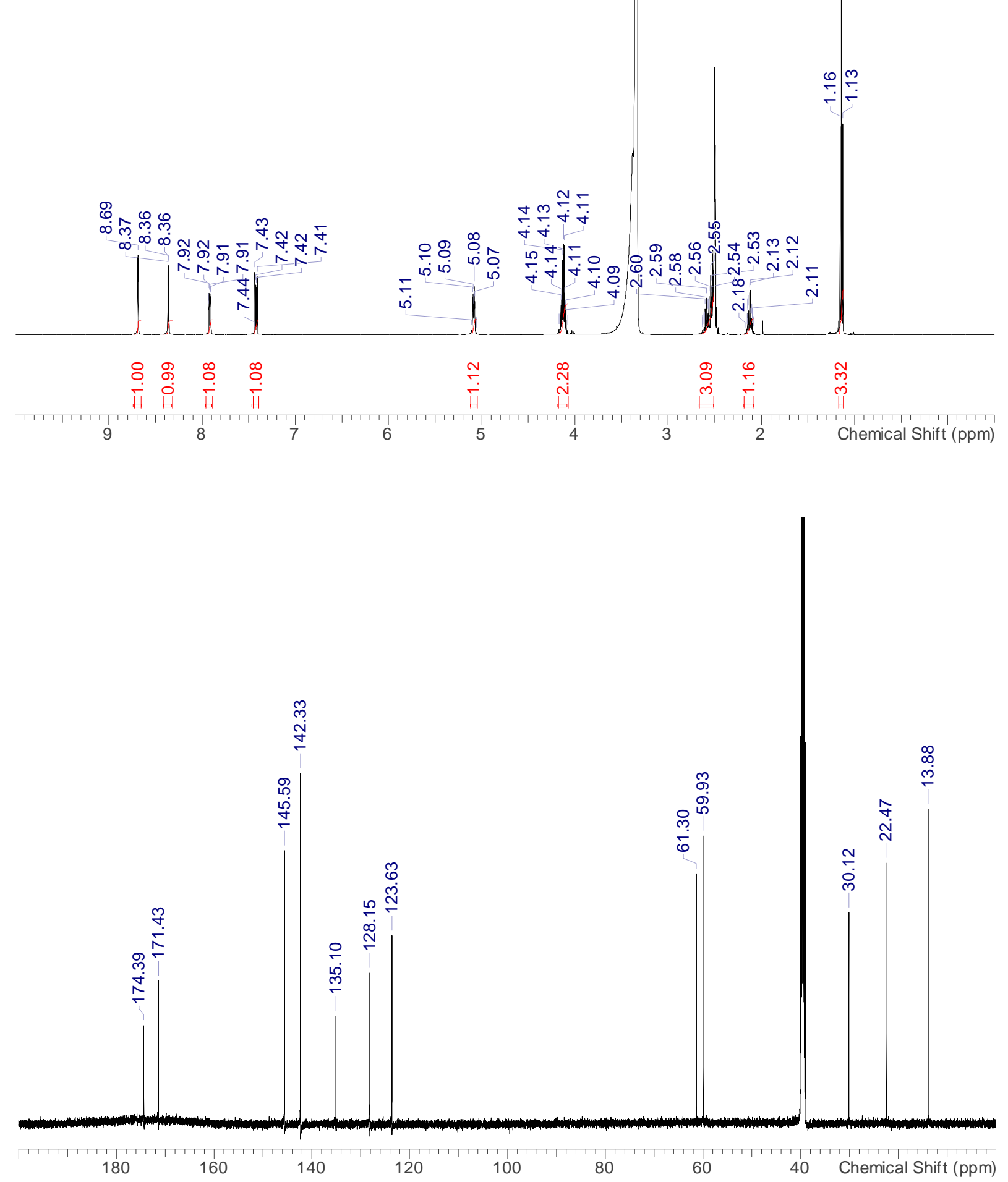
Page S55<smiles>CCOC(=O)[C@@H]1CCC(=O)N1c1ccccn1</smiles>

$\stackrel{\infty}{\infty}$

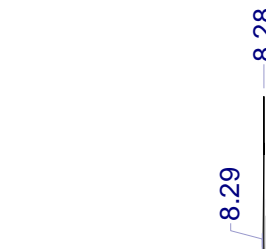

ヘิ

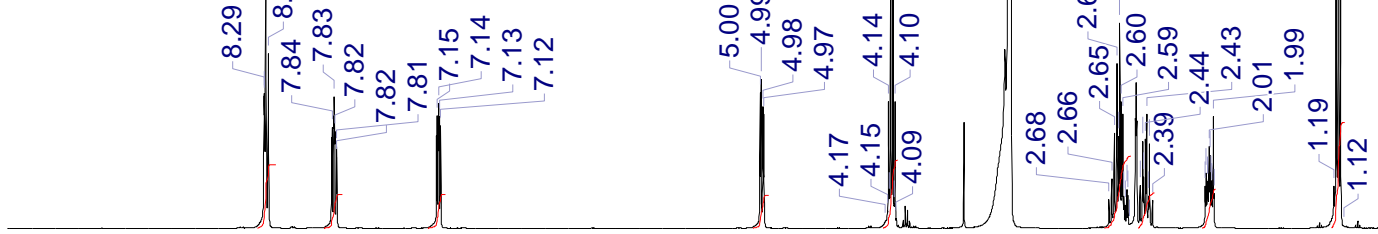

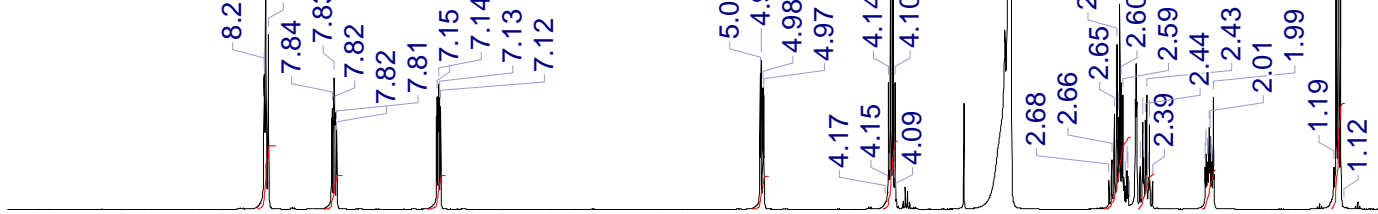
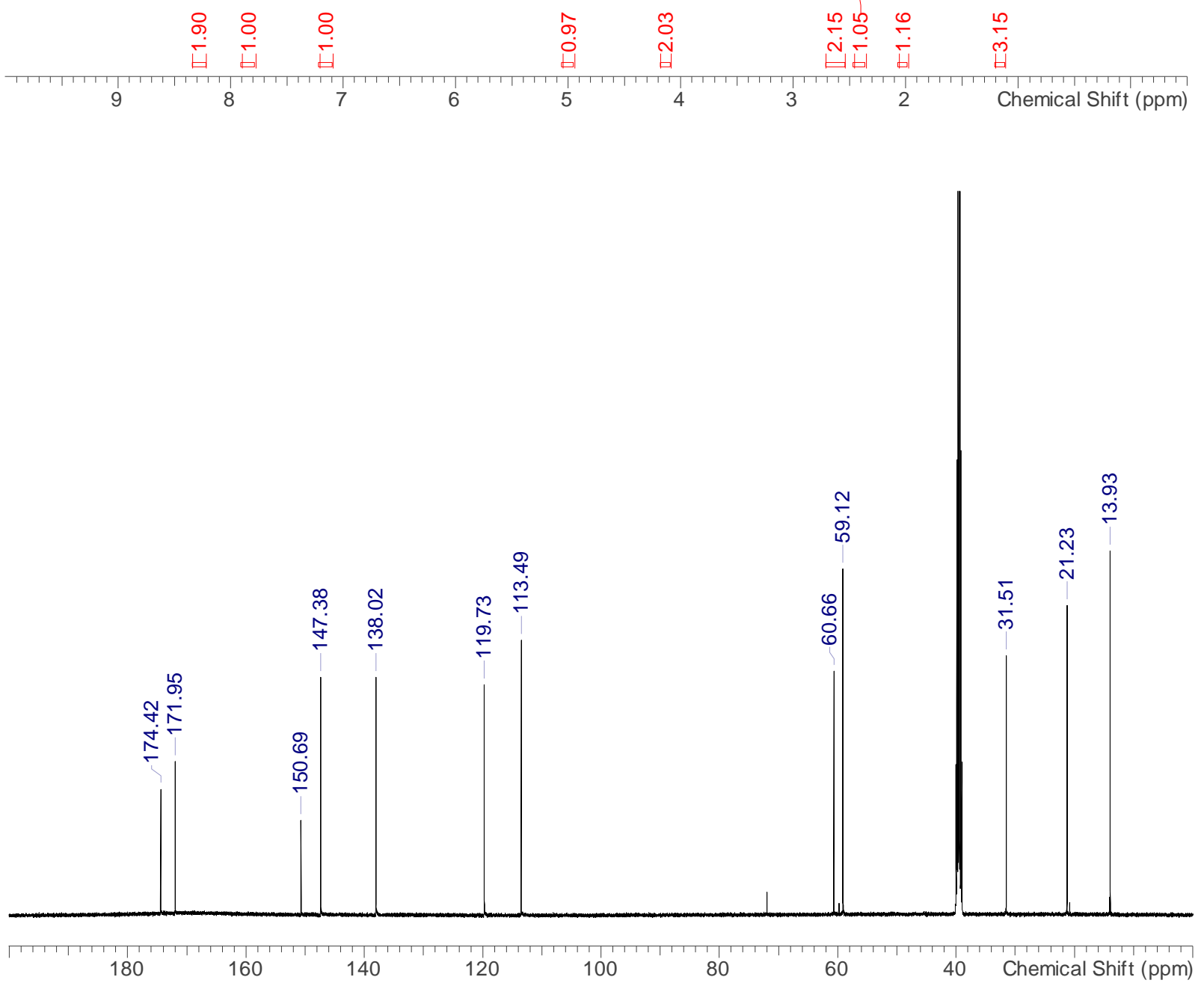
Page S56

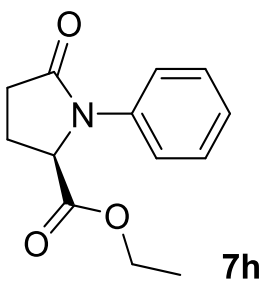

Water
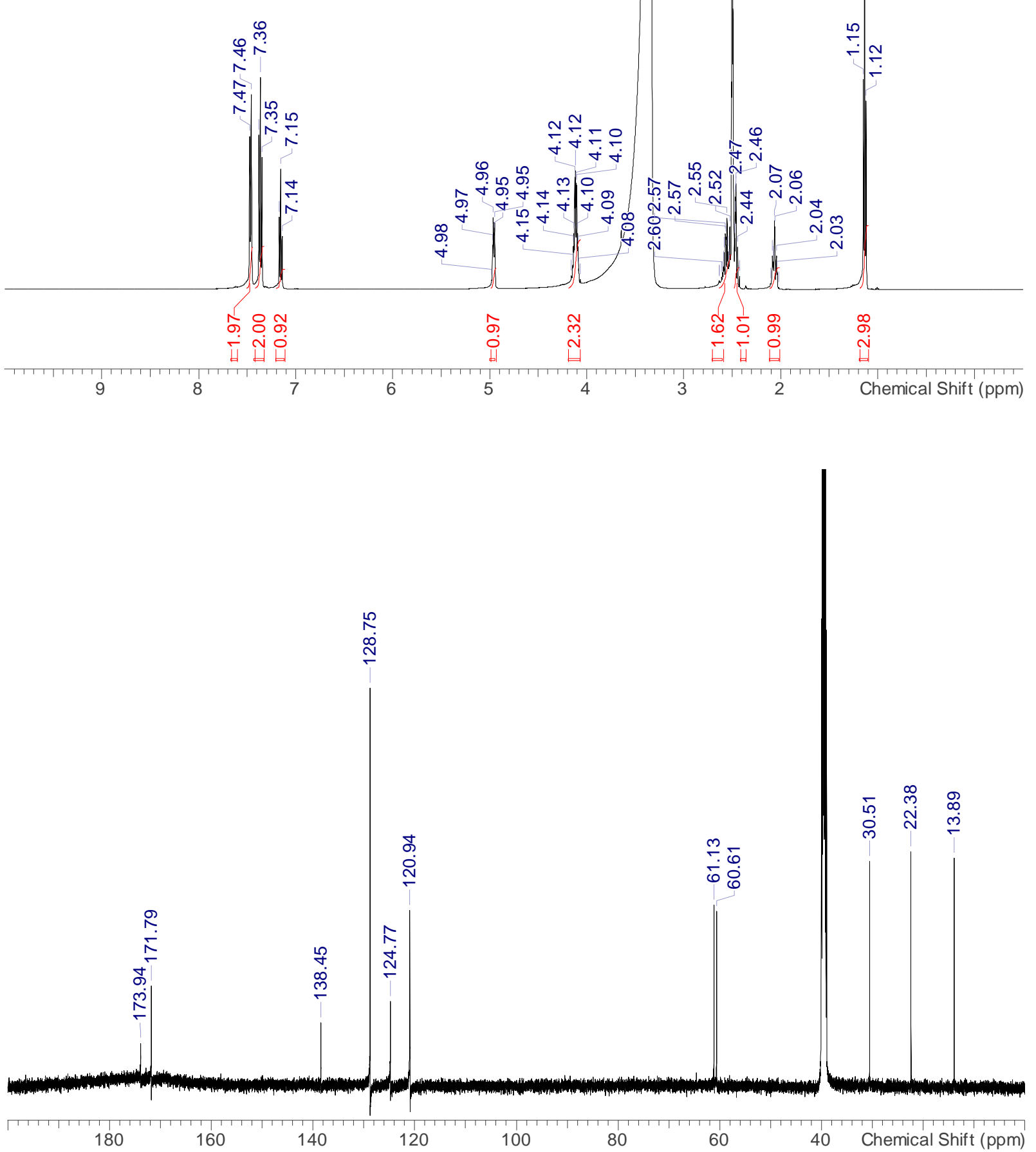
Page S57
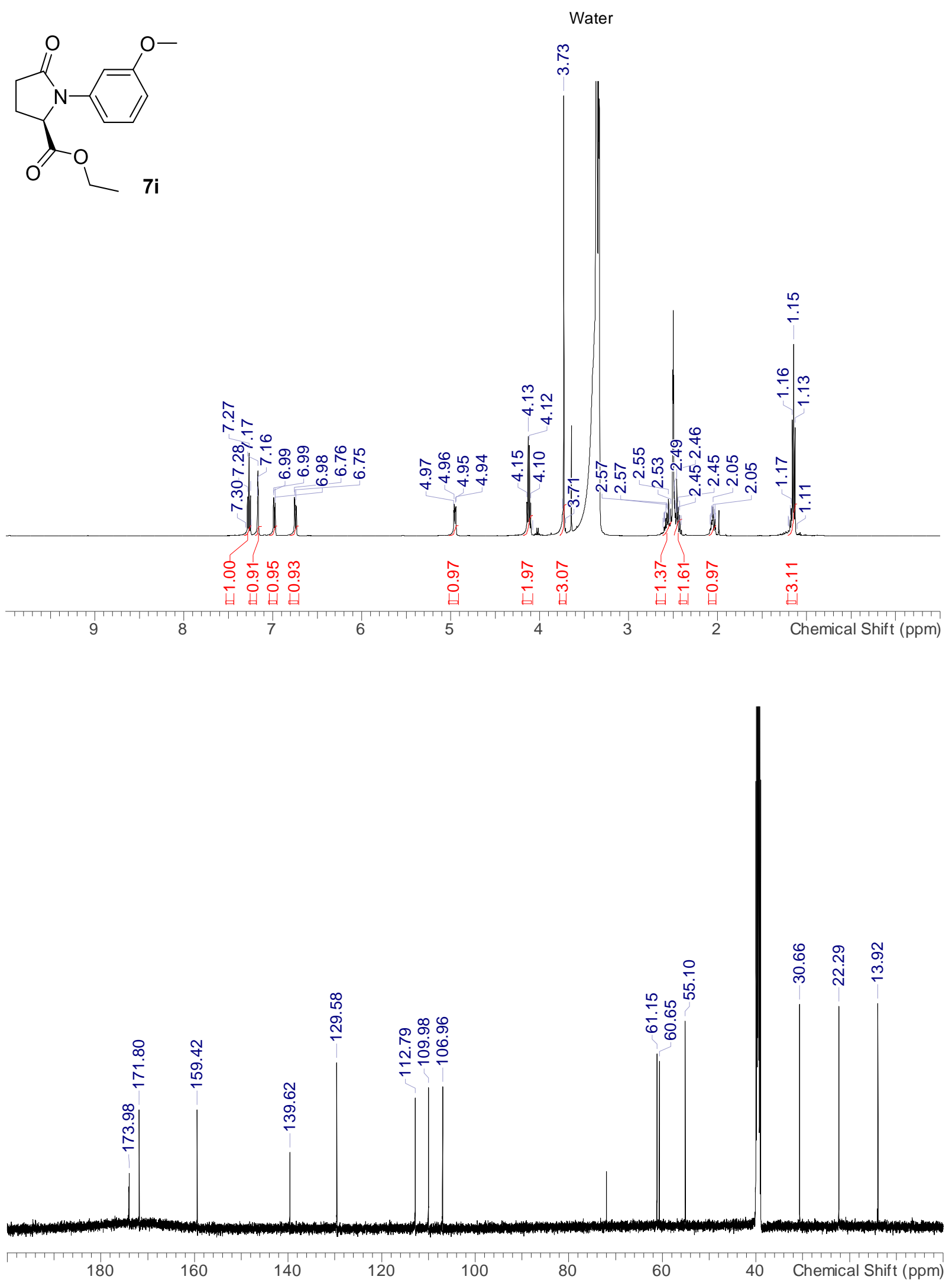
Page S58<smiles>CCOC(=O)[C@@H]1CCC(=O)N1c1ccccc1OC</smiles>

$\stackrel{\infty}{\wedge}$

Water
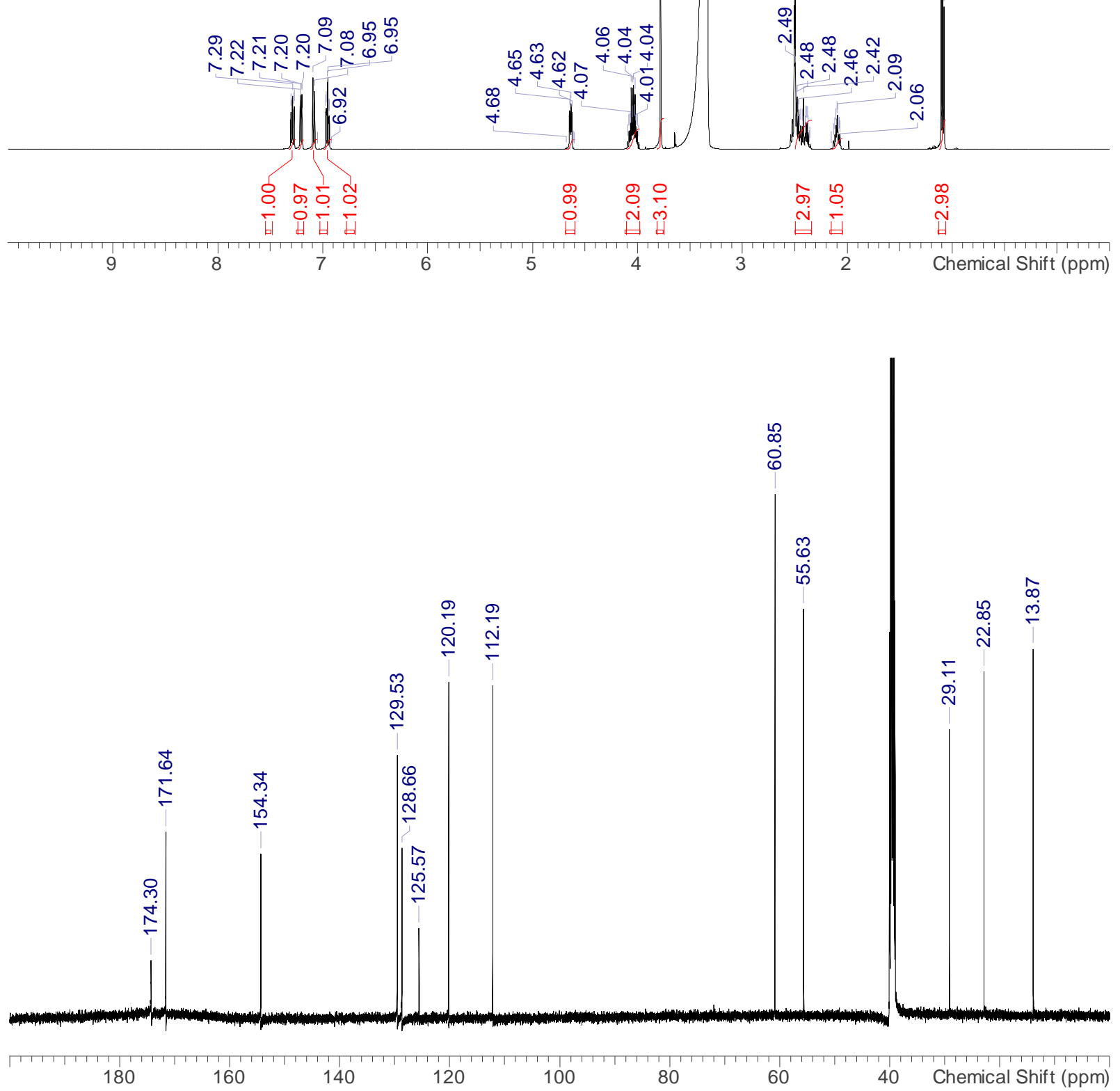
Page S59<smiles>COc1ccc(CN2CCC[C@H](Nc3ccc(C(F)(F)F)cc3)C2=O)cc1</smiles>
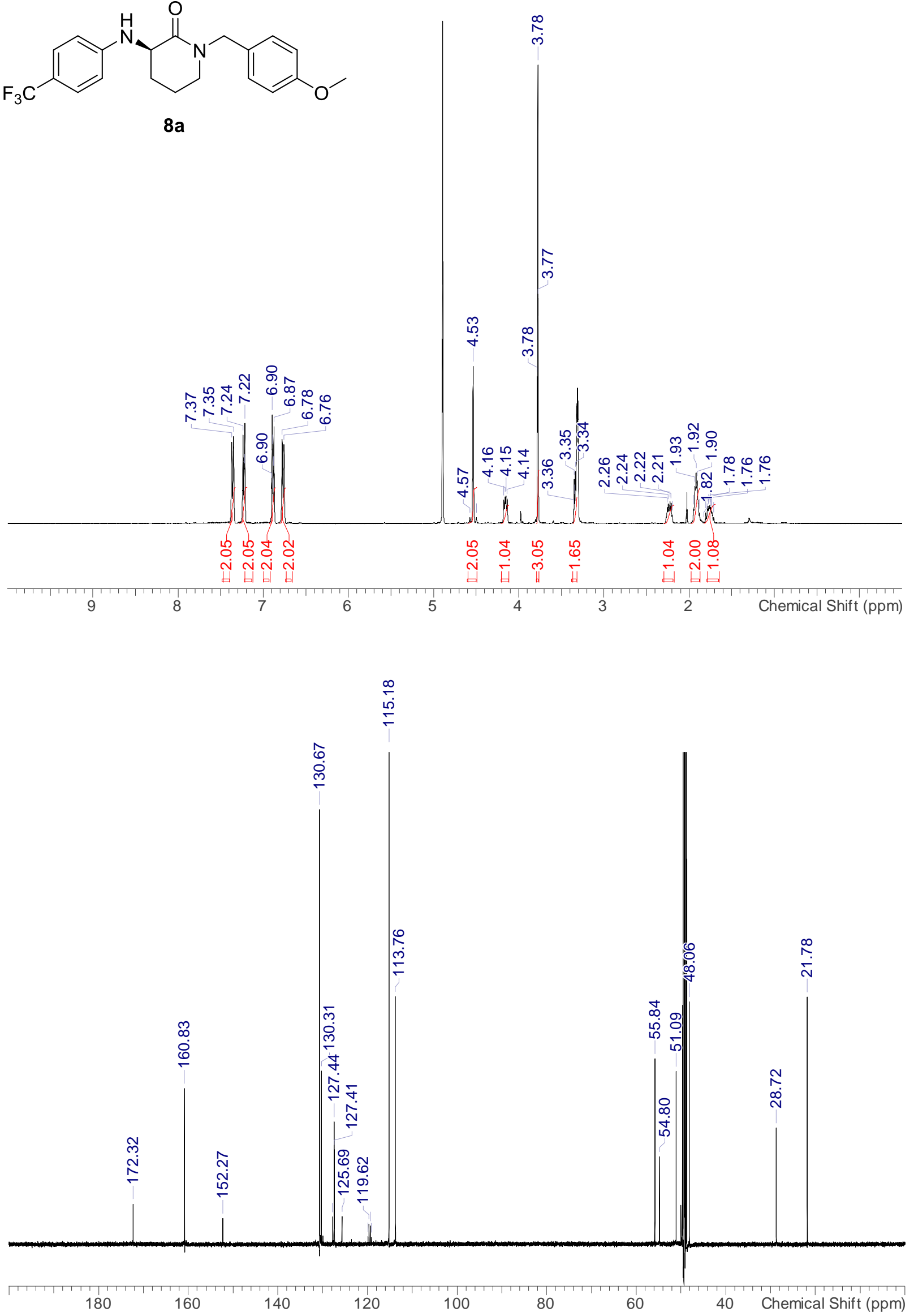
Page S60<smiles>COc1ccc(CN2CCC[C@H](Nc3cccc(C(F)(F)F)c3)C2=O)cc1</smiles>
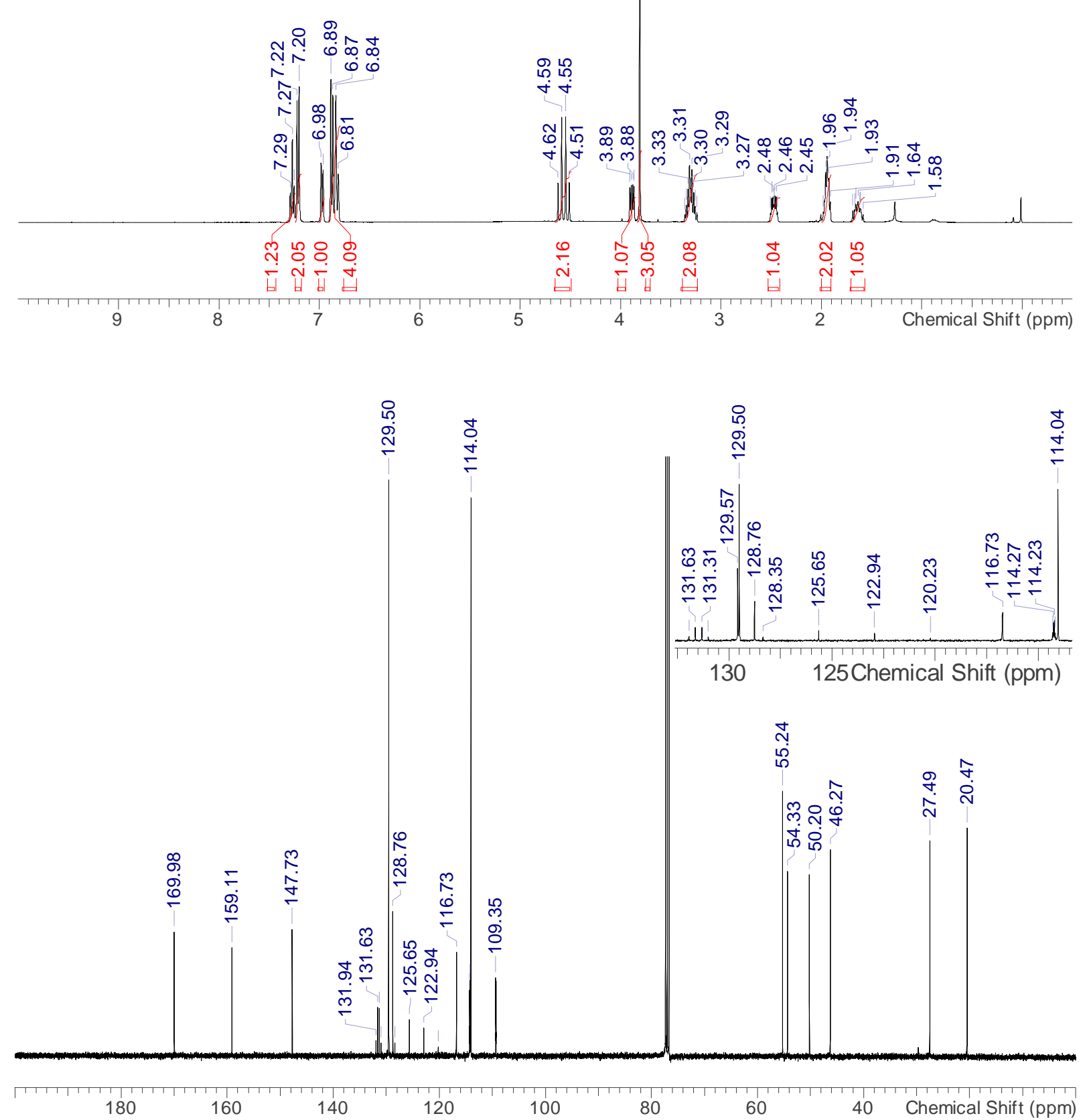
Page S61<smiles>COc1ccc(CN2CCC[C@H](Nc3cc(F)cc(C(F)(F)F)c3)C2=O)cc1</smiles>
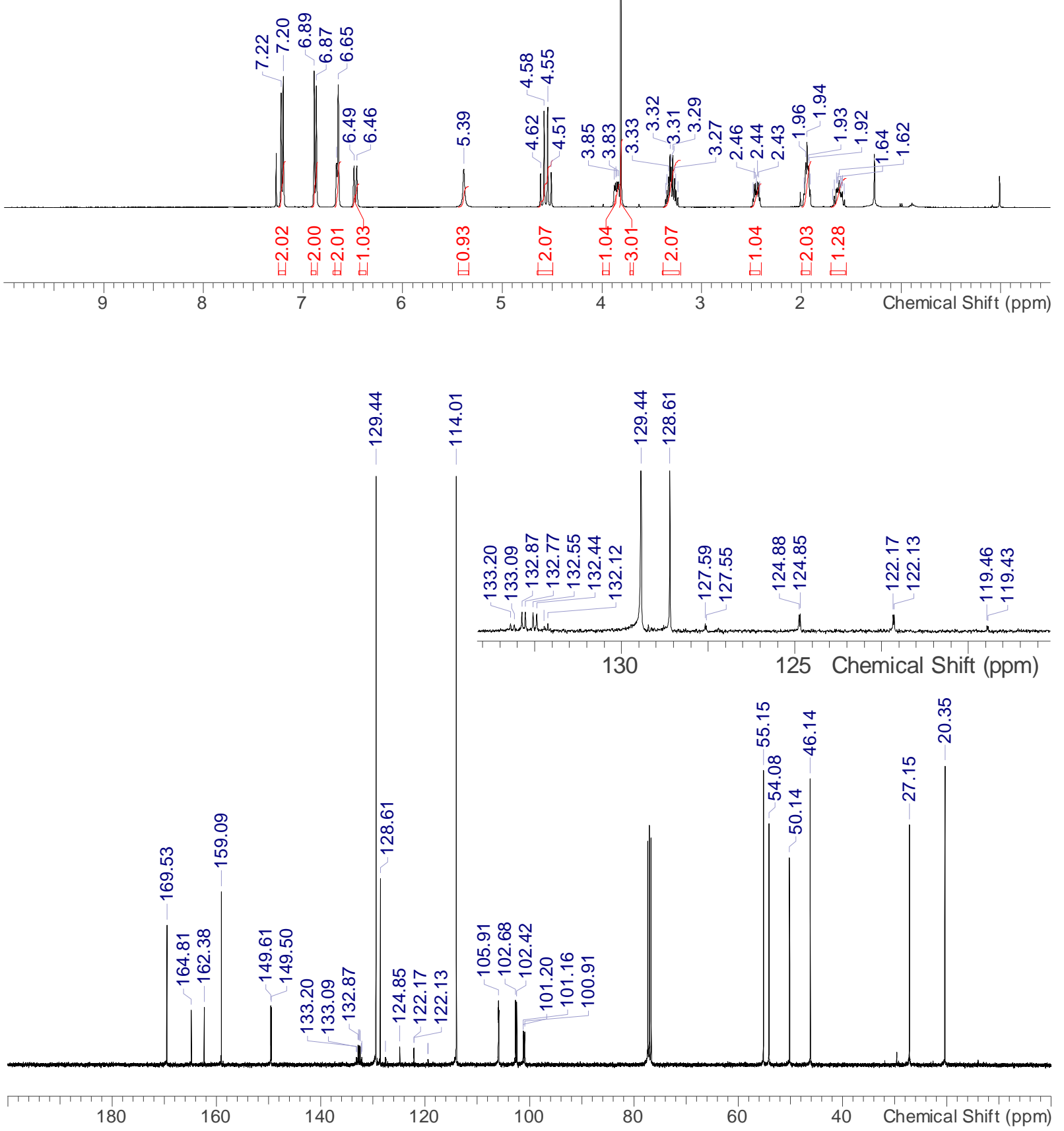
Page S62<smiles>COc1ccc(CN2CCC[C@H](Nc3cc(F)cc(Cl)c3)C2=O)cc1</smiles>
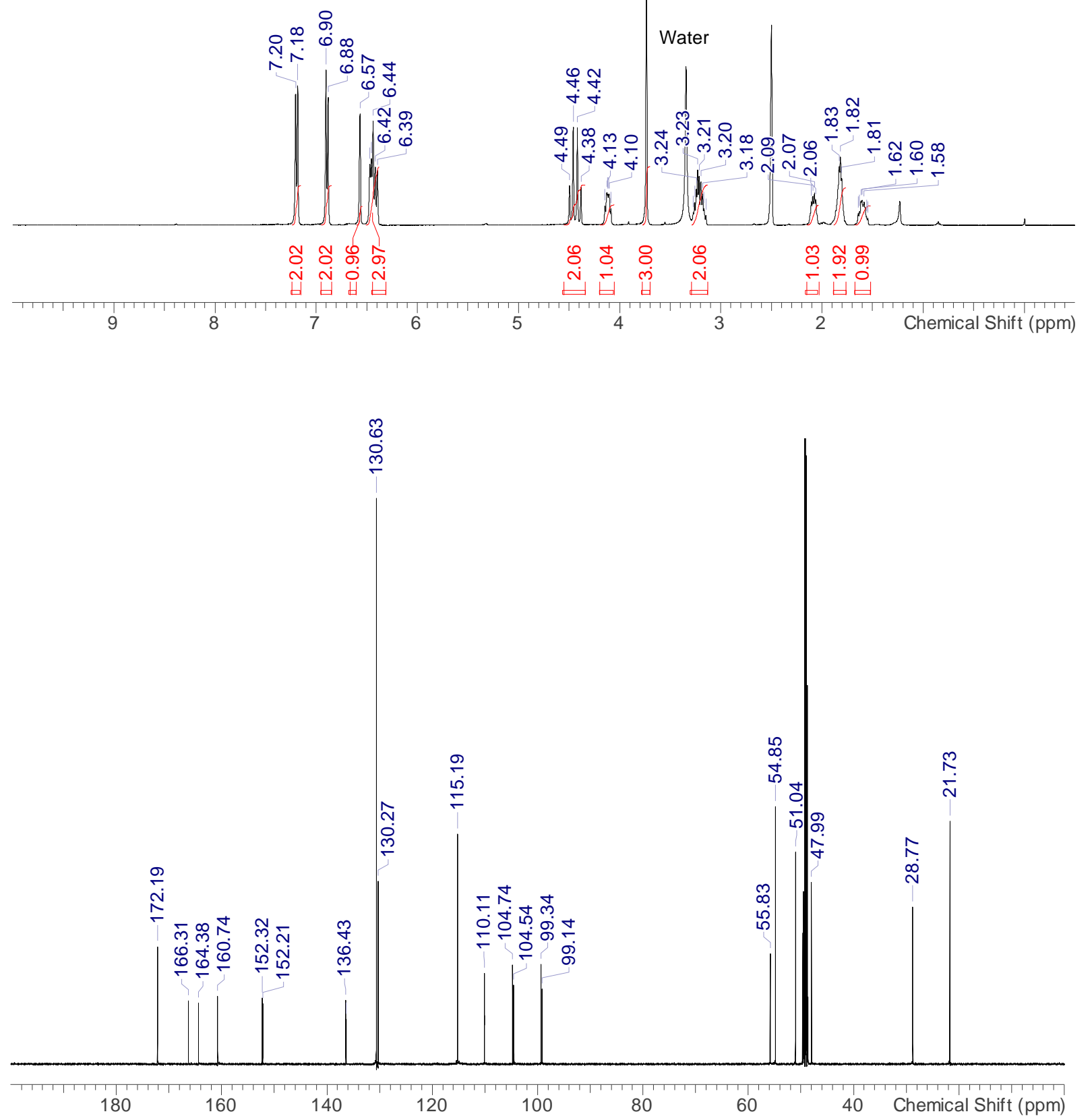
Page S63<smiles>COc1ccc(CN2CCC[C@H](Nc3ccncc3)C2=O)cc1</smiles>
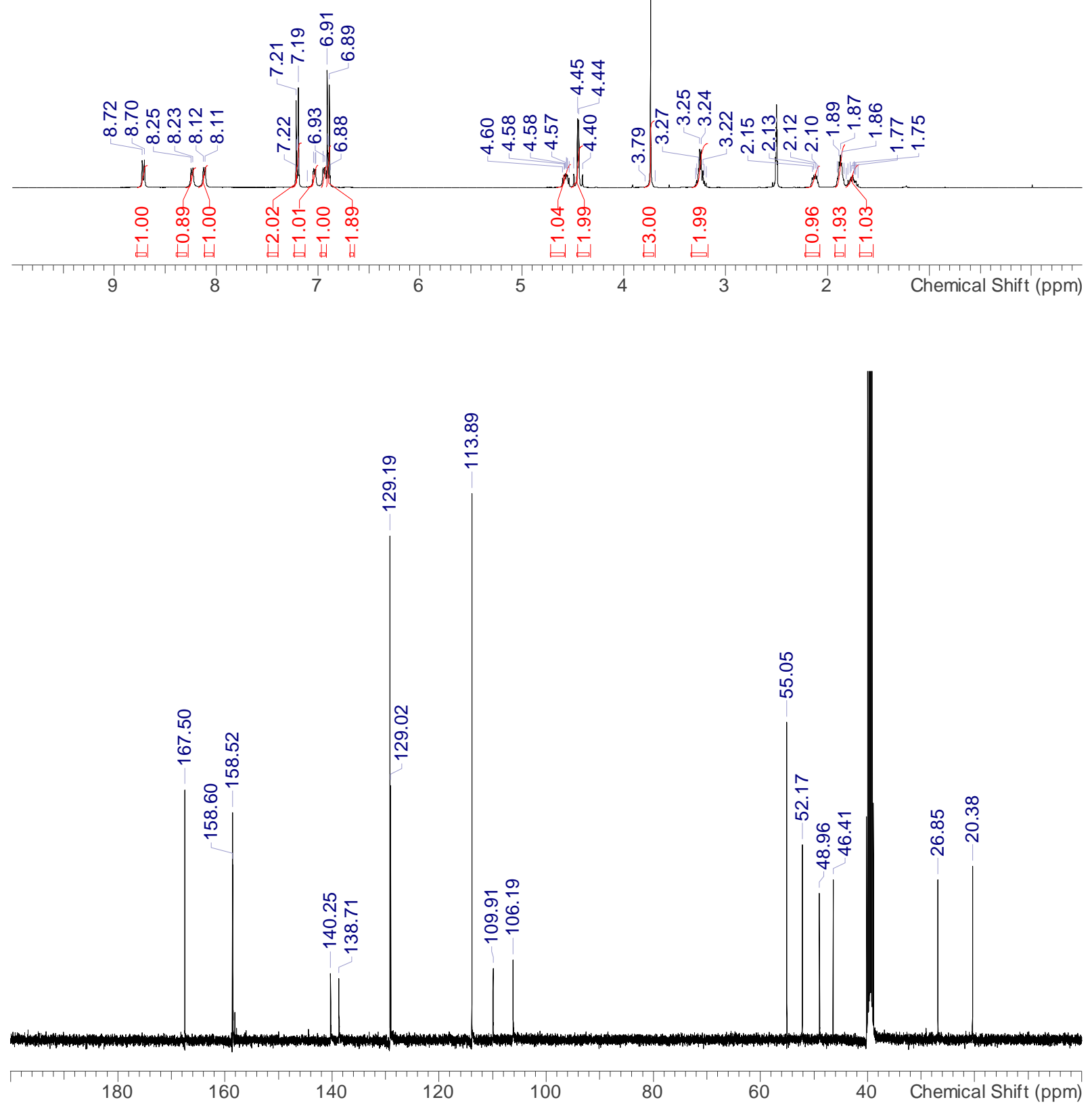
Page S64
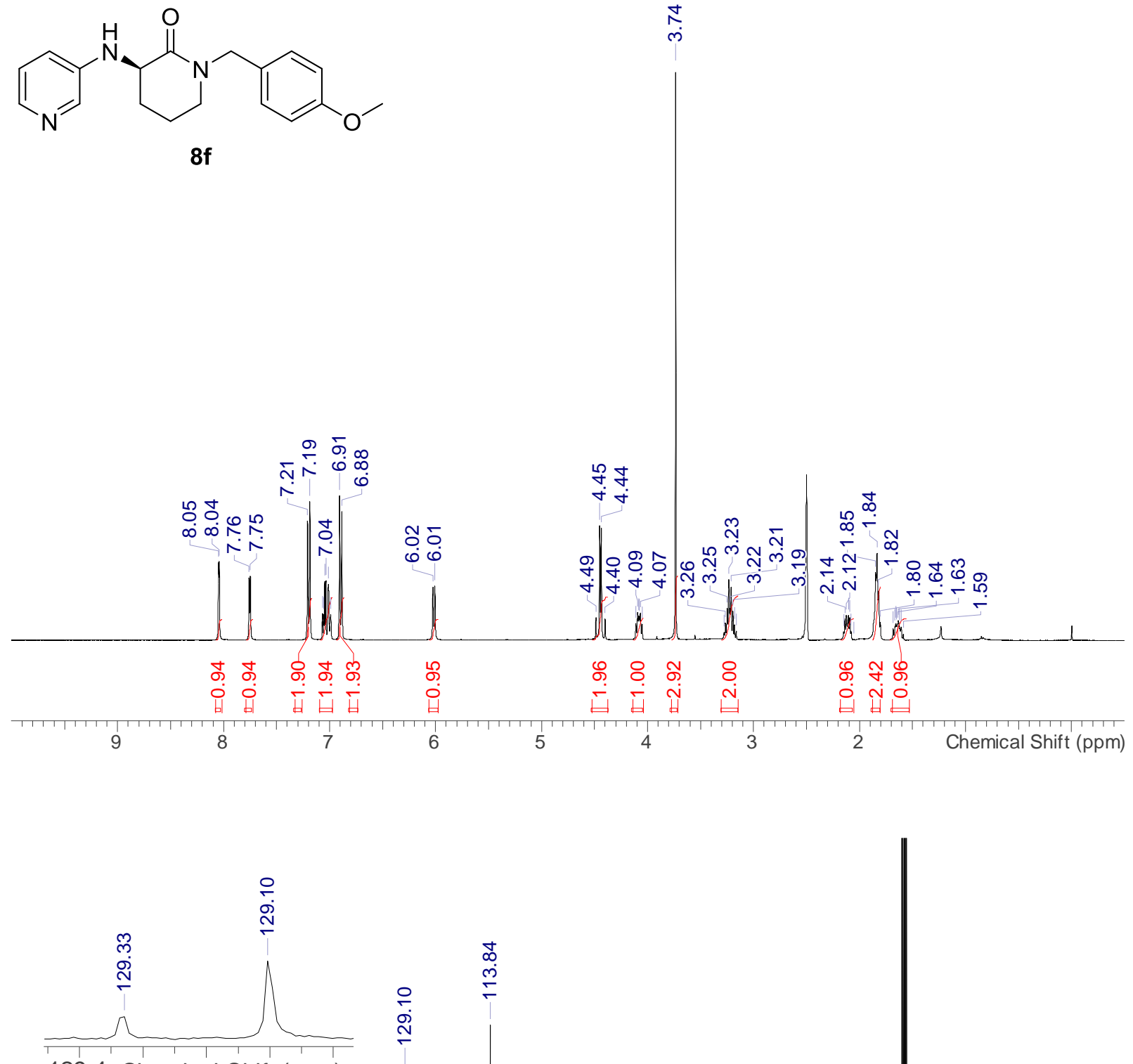

129.4 Chemical Shift (ppm)
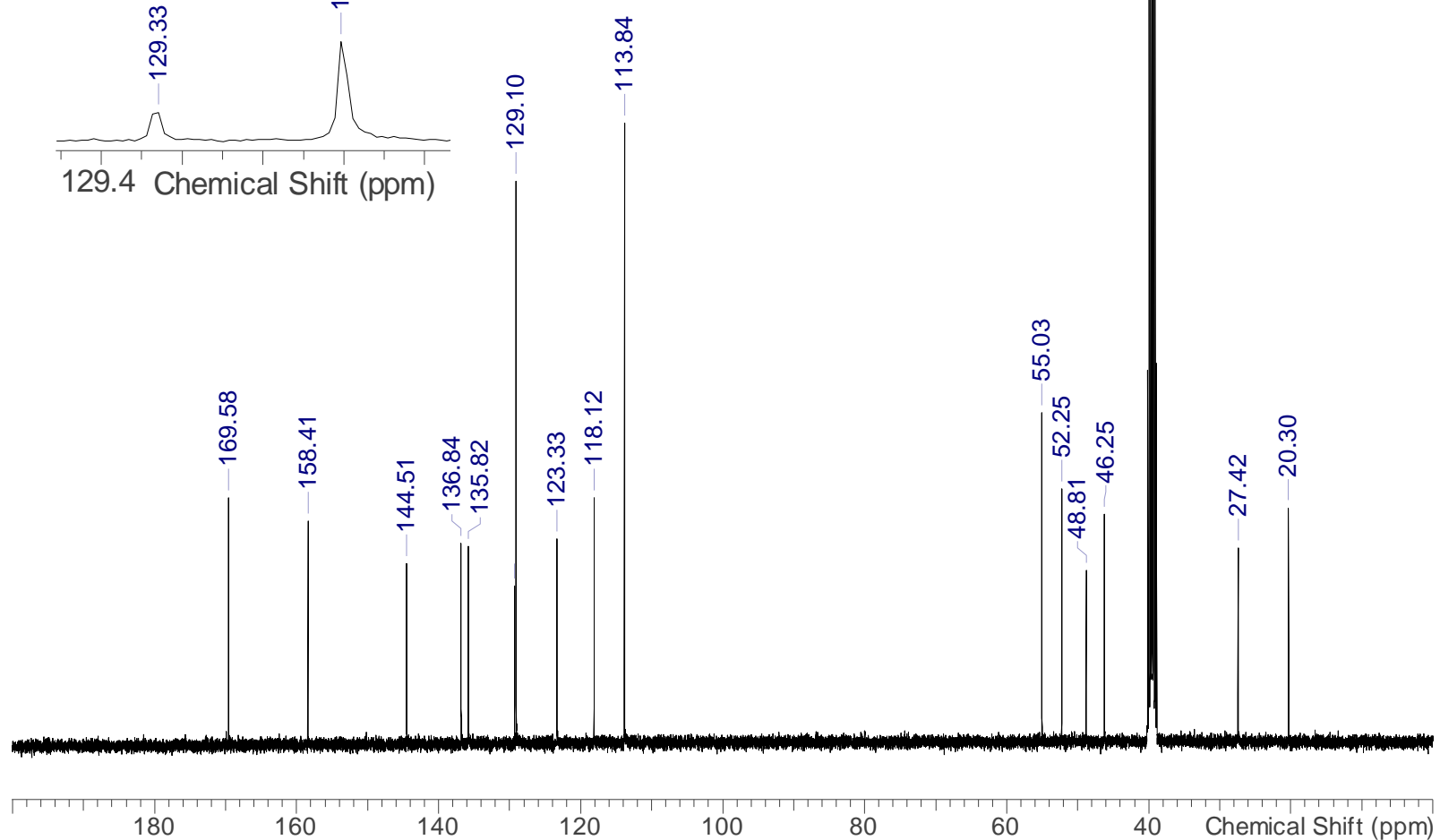
Page S65<smiles>COc1ccc(CN2CCC[C@H](Nc3ccccn3)C2=O)cc1</smiles>

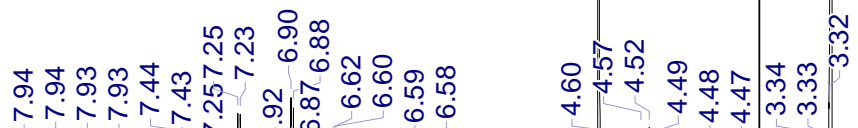
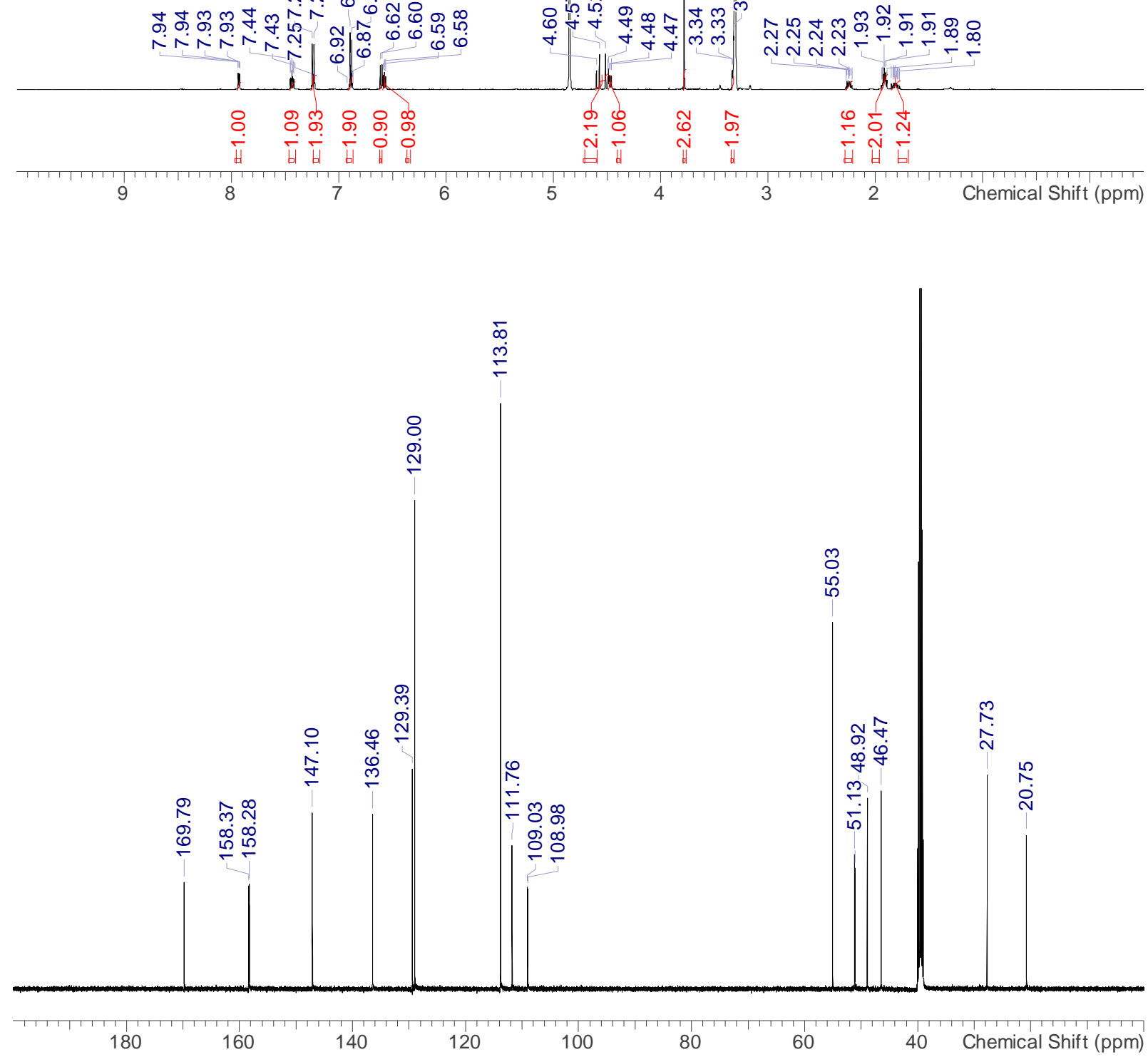
Page S66<smiles>COc1ccc(CN2CCC[C@H](Nc3ccccc3)C2=O)cc1</smiles>

성
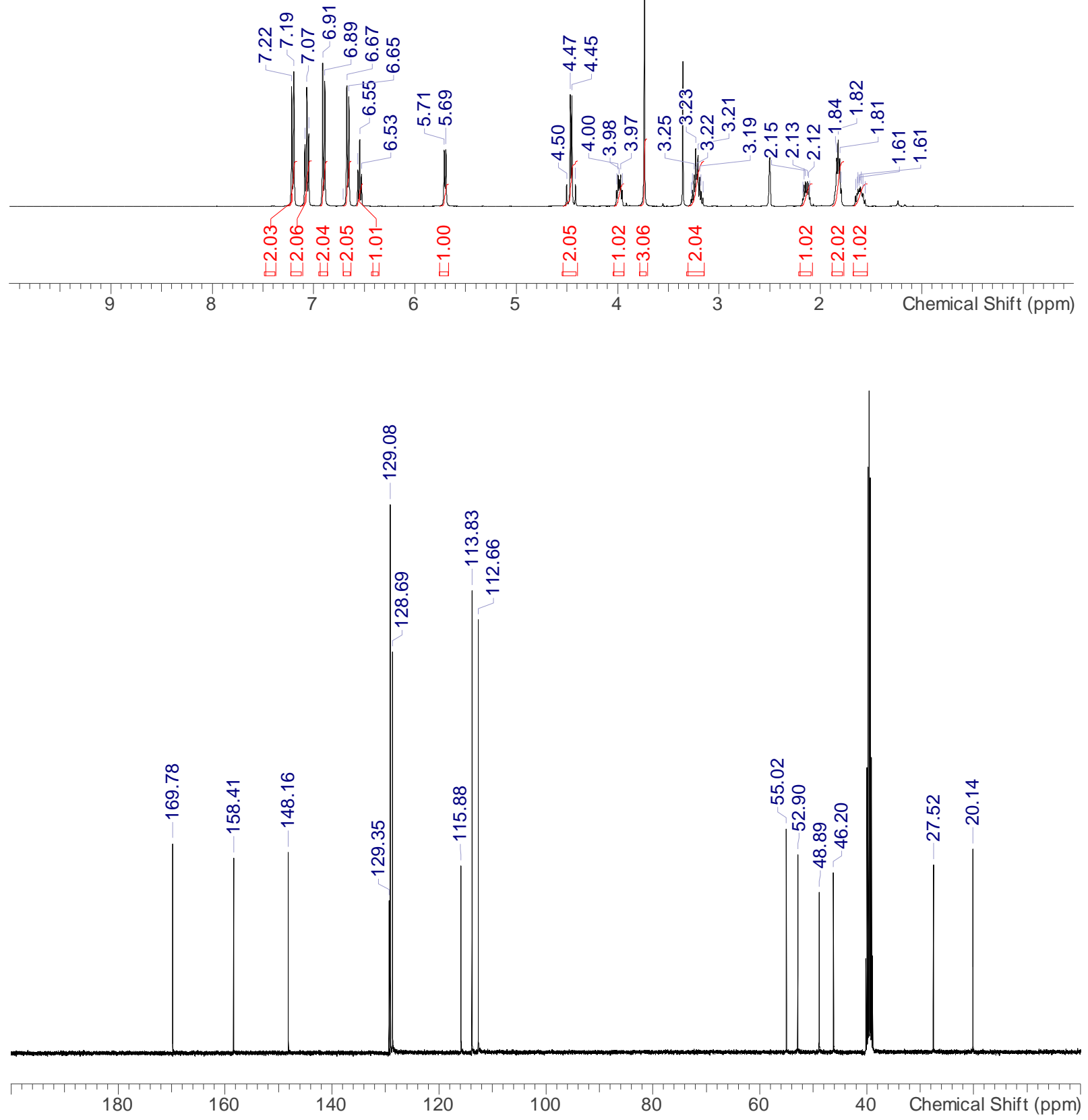

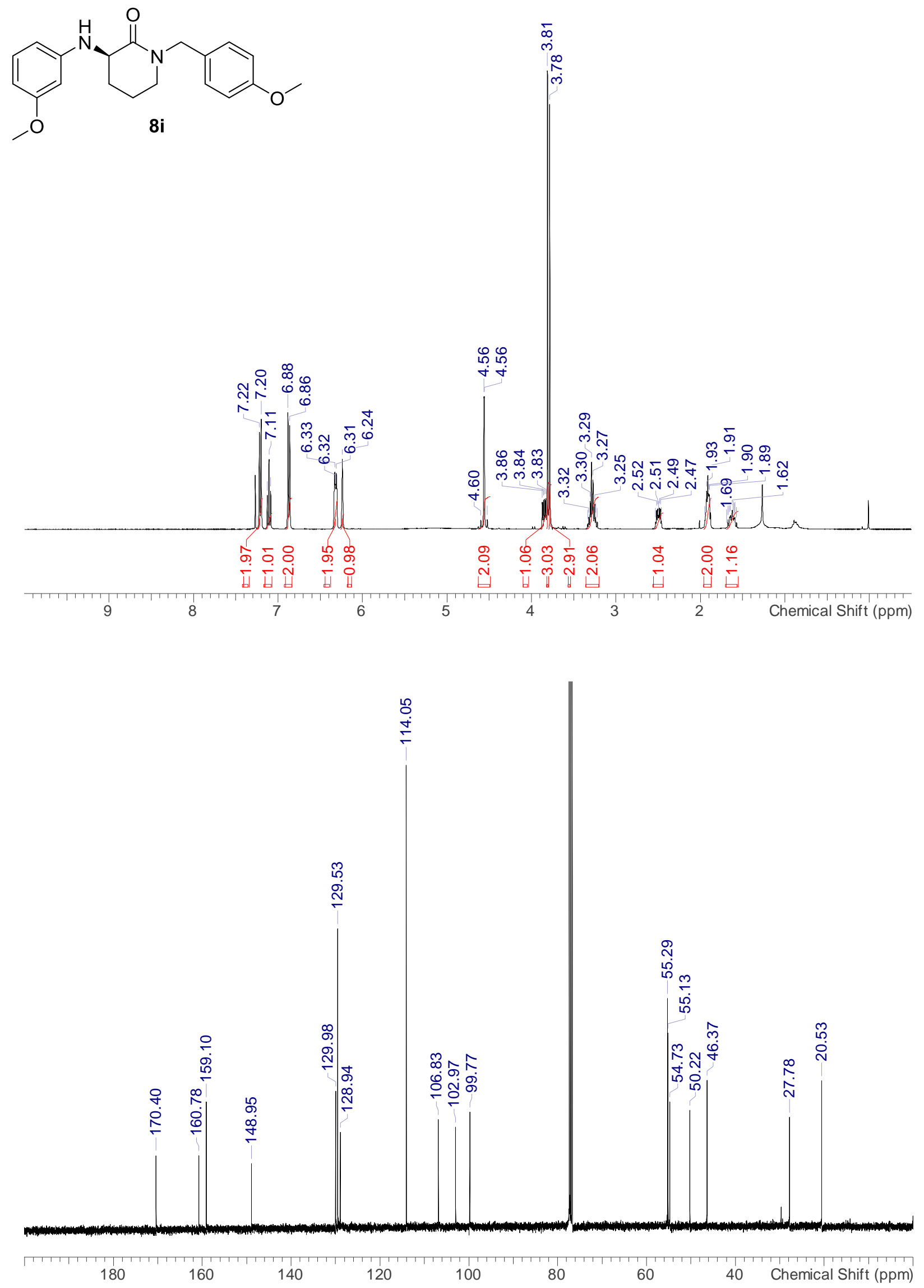
Page S68<smiles>COc1ccc(CN2CCC[C@H](Nc3ccccc3OC)C2=O)cc1</smiles>

8j

$\infty$
$\infty$
$\infty$

j
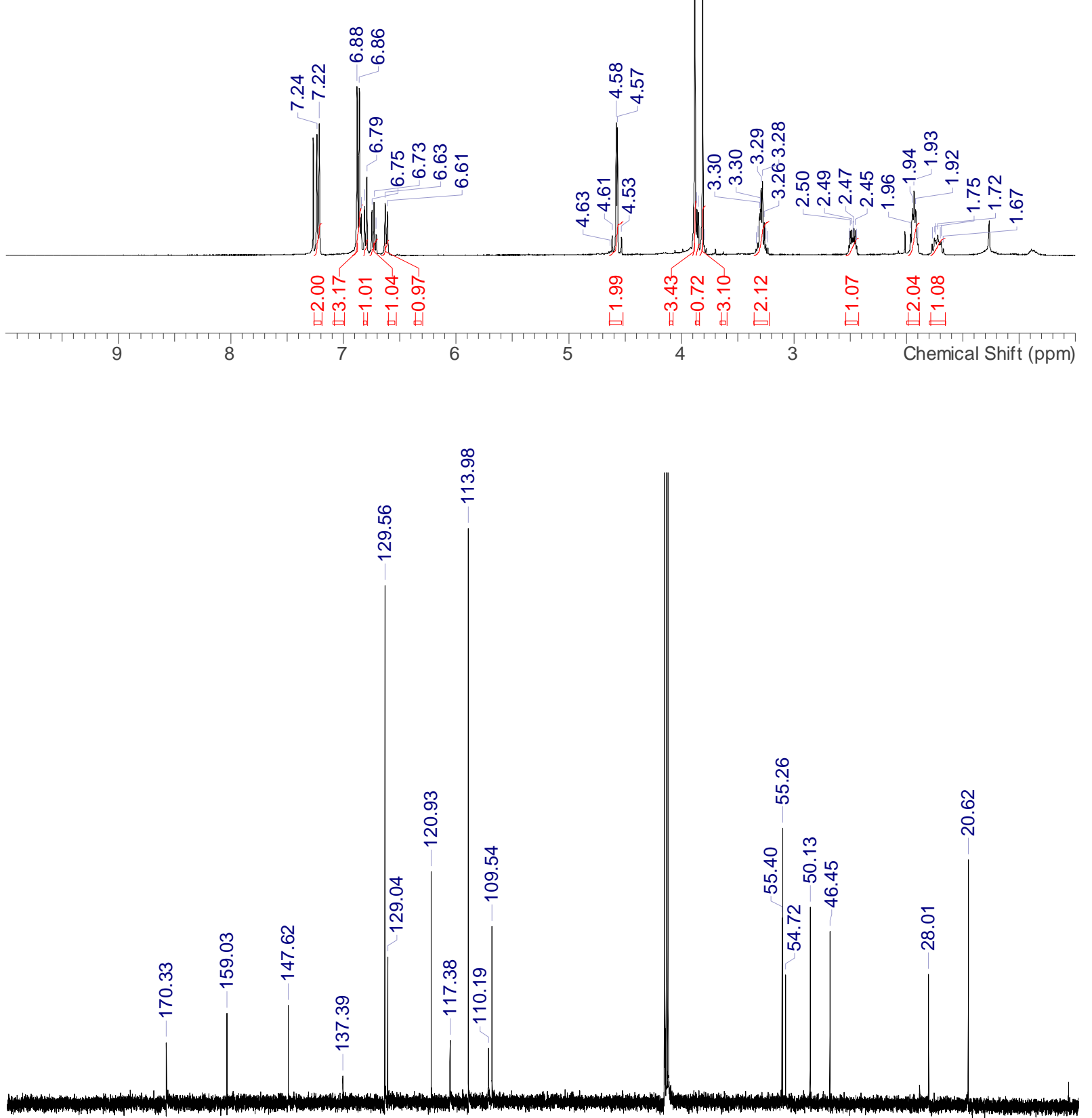
Page S69<smiles>CCOC(=O)[C@H](CCCNCc1ccc(OC)cc1)Nc1ccc(C(F)(F)F)cc1</smiles>

9
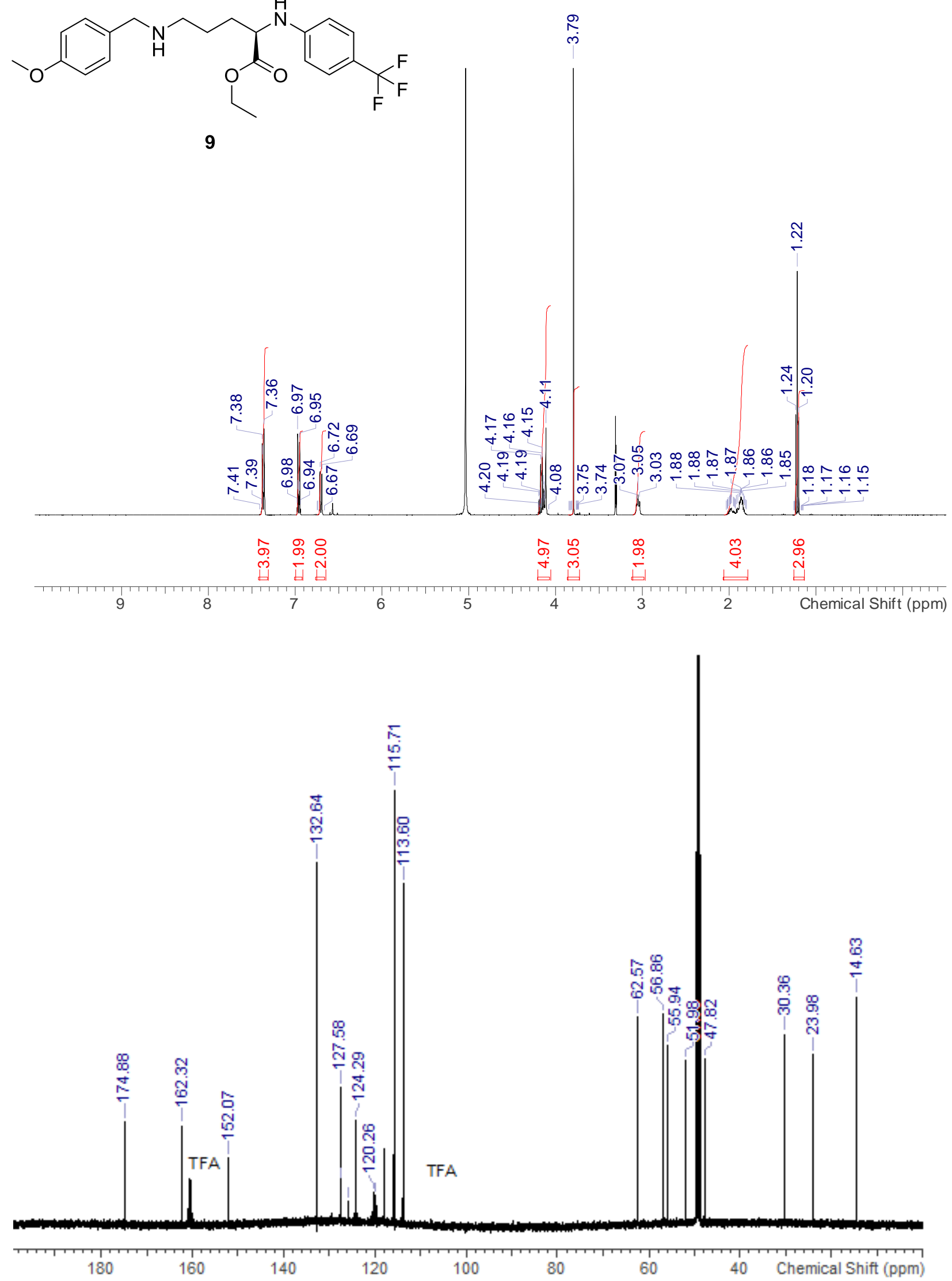